\title{
Teorema de Serre-Swan para grupoides de Lie étale
}

\author{
Jackeline Conrado
}

\author{
DisSERTAÇÃO APRESENTADA \\ $\mathrm{AO}$ \\ Instituto De Matemática e Estatística \\ DA \\ Universidade De SÃo Paulo \\ PARA \\ OBTENÇÃO DO TÍTULO \\ $\mathrm{DE}$ \\ Mestre em Matemática
}

Programa: Matemática

Orientador: Prof. Dr. Cristián Ortiz

Durante o desenvolvimento deste trabalho o autor recebeu auxílio financeiro parcial do CNPq.

São Paulo, Dezembro de 2016. 


\section{Teorema de Serre-Swan para grupoides de Lie étale}

Esta é a versão original da dissertação elaborada pela candidata Jackeline Conrado, tal como submetida à Comissão Julgadora. 


\section{Teorema de Serre-Swan para grupoides de Lie étale}

Esta versão da dissertação contém as correções e alterações sugeridas pela Comissão Julgadora durante a defesa da versão original do trabalho, realizada em 12/12/2016. Uma cópia da versão original está disponível no Instituto de Matemática e Estatística da Universidade de São Paulo.

Comissão Julgadora:

- Prof. Dr. Cristián Ortiz - IME-USP

- Prof. Dr. Olivier Brahic - DMAT-UFPR

- Prof. Dr. Lino Grama - IMECC-UNICAMP 


\section{Agradecimentos}

Agradeço a minha família pelo apoio e incentivo. Aos meus amigos, que ao longo destes dois anos fizeram parte da minha vida, compartilhando ideias e me tornando uma pessoa melhor. Ao meu orientador Cristián e à banca examinadora pelas correções e sugestões. Agradeço ao CNPq, pelo apoio financeiro. 


\section{Resumo}

CONRADO, J. Teorema de Serre-Swan para grupoides de Lie étale. Dissertação (Mestrado) - Instituto de Matemática e Estatística, Universidade de São Paulo, São Paulo, 2016.

Este trabalho tem dois objetivos principais. O primeiro é estender o Teorema de Serre-Swan para grupoides de Lie étale. O segundo é demonstrar que, se dois grupoides de Lie étale são Morita equivalentes então a categoria dos módulos sobre as álgebras de convolução destes grupoides são equivalentes, e esta equivalência preserva a subcategoria dos módulos de tipo finito e posto constante.

Palavras-chave: grupoides de Lie étale, Serre-Swan, álgebra de convolução, equivalência de Morita. 


\section{Abstract}

CONRADO, J. Teorema de Serre-Swan para grupoides de Lie étale. Dissertação (Mestrado)

- Instituto de Matemática e Estatística, Universidade de São Paulo, São Paulo, 2016.

In this work we have two main goals. The first one is to extend the Serre-Swan's theorem. Our second goal is to prove, if two étale Lie groupoids are Morita equivalence then the category of modules over its convolution algebra are Morita equivalence, and this equivalence preserve the subcategory of modules of finite type and of constant rank.

Keywords: étale Lie groupoid, Serre-Swan, convolution algebra, Morita equivalence. 


\section{Sumário}

1 Teorema de Serre-Swan 5

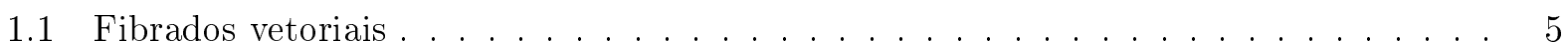

1.2 Morfismos de fibrados vetoriais $\ldots \ldots \ldots \ldots \ldots$

1.3 Módulos projetivos e módulos finitamente gerados $\ldots \ldots \ldots \ldots$. . . . . . . 9

1.4 Teorema de Serre-Swan . . . . . . . . . . . . . . . . . . . 11

2 Grupoides de Lie $\quad 15$

2.1 Grupoides: definições e exemplos. . . . . . . . . . . . . . . . . . . . 15

2.2 Grupoides de Lie étale . . . . . . . . . . . . . . . . . . . . . . 17

2.3 Representações de grupoides de Lie . . . . . . . . . . . . . . . . . . . 18

2.4 Equivalência de Morita . . . . . . . . . . . . . . . . . . . . . . 23

2.4 .1 Invariância de Morita para representações . . . . . . . . . . . . . . . . . . 29

3 Teorema de Serre-Swan para grupoides de Lie étale 33

3.1 Álgebra de convolução de um grupoide de Lie étale . . . . . . . . . . . . . . . . . 33

3.2 Módulos de tipo finito e módulos de posto constante . . . . . . . . . . . . . . 37

3.3 Teorema de Serre-Swan para grupoides de Lie étale . . . . . . . . . . . . . . . . . 44

4 Equivalência de Morita para álgebras de convolução $\quad 51$

4.1 Equivalência de Morita e a categoria dos módulos . . . . . . . . . . . . . . . . 51

4.2 Equivalência para módulos de tipo finito e posto constante . . . . . . . . . . . 57

$\begin{array}{ll}\text { Referências Bibliográficas } & 61\end{array}$ 


\section{Introdução}

O uso de ferramentas algébricas é fundamental para compreendermos determinadas propriedades geométricas em variedades. Um dos teoremas nesse contexto, é o Teorema de Gelfand-Naimark que estabelece uma equivalência entre a categoria dos espaços topológicos localmente compactos e a categoria das álgebras associativas e comutativas dada por

$$
X \longmapsto C_{c}^{o}(X),
$$

que associa a cada espaço a álgebra de funções contínuas com suporte compacto, com as operações definidas ponto a ponto.

Além da correspondência entre espaços topológicos e álgebras associativas e comutativas, podemos procurar correspondências entre estruturas geométricas mais ricas, ou seja, para estudarmos propriedades geométricas de variedades diferenciáveis, fazemos uso de ferramentas tais como: campos de vetores, formas diferenciais, classes características, entre outras. Todas estas ferramentas dependem da noção de fibrado vetorial. Desta forma, dado um fibrado vetorial $E$ sobre uma variedade diferenciável $M$, é natural perguntarmos qual é o conceito algébrico correspondente. A resposta para essa pergunta é o seguinte resultado.

(Teorema de Serre-Swan) Seja $M$ uma variedade diferenciável. Considere as categorias $\operatorname{Vect}(M)$ e $\operatorname{Mod}_{p f g}(M)$ dos fibrados vetoriais sobre $M$ e $\operatorname{dos} C_{c}^{\infty}(M)$-módulos projetivos e finitamente gerados. O funtor

$$
\begin{aligned}
\Gamma_{c}^{\infty}: \operatorname{Vect}(M) & \longrightarrow \operatorname{Mod}_{p f g}(M) \\
E \longmapsto & \longrightarrow \Gamma_{c}^{\infty}(E)
\end{aligned}
$$

é uma equivalência de categorias.

Outra ferramenta importante no estudo de variedades é dada por simetrias, por exemplo, ações de grupos de Lie. Neste contexto, o correspondente espaço quociente contém informação geométrica importante. Porém, quocientes de variedades diferenciáveis em geral são singulares (e.g. orbifolds).

Por outro lado, quando retiramos algumas das hipóteses acima, ou seja, quando temos uma uma ação que não é própria ou não é livre, então o espaço quociente poderá ter singularidades. Na tentativa de estudar estes espaços singulares, podemos estudar espaços suaves que contêm informação singular. Tais espaços são chamados de Grupoides de Lie. Um grupoide é uma categoria pequena onde todos os morfismos são invertíveis. Assim, um grupoide consiste em um conjunto de objetos $M$, um conjunto de morfismos entre objetos (ou flechas) $G$, e mapas estruturais s(source), $\mathrm{t}$ (target), m(multiplicação parcialmente definida), $\epsilon$ (seção identidade) e $i$ (inversão), satisfazendo axiomas de uma categoria. Dizemos que $G \rightrightarrows M$ é um grupoide de Lie, se $G$ e $M$ são variedades diferenciáveis, os mapas estruturais são suaves e $s, t: G \rightarrow M$ são submersões sobrejetoras. 
É conhecido que toda ação de um grupo de Lie em uma variedade diferenciável define um grupoide de Lie $G \ltimes M \rightrightarrows M$, conhecido como grupoide de transformação. Além deste exemplo, temos que toda variedade diferenciável $M$ define um grupoide $M \rightrightarrows M$. Este grupoide é um grupoide de Lie étale ( i.e. $\operatorname{dim} G=\operatorname{dim} M$ ). Assim, temos uma inclusão natural da categoria das variedades diferenciáveis na categoria dos grupoides de Lie étale dada por:

$$
\begin{aligned}
\text { Variedades diferenciáveis } & \longrightarrow \text { Grupoides de Lie étale } \\
M & \longmapsto M \rightrightarrows M
\end{aligned}
$$

Todo grupoide de Lie $G \rightrightarrows M$ tem associado um espaço de órbitas $M / G$. No caso do grupoide de transformação o espaço de órbitas recupera o espaço de órbitas da ação. Assim, para entender a geometria de variedades com simetrias, torna-se importante estudar grupoides de Lie e fibrados vetoriais sobre grupoides de Lie. Neste trabalho nos concentramos no caso de grupoides de Lie étale. De forma precisa, apresentamos extensões naturais do Teorema de Gelfand-Naimark e do Teorema de Serre-Swan para grupoides de Lie étale.

Para incluir o exemplo, o qual $G \ltimes M \rightrightarrows M$ não é étale, é importante entender como as construções da álgebra de convolução de um grupoide $G$, e da sua categoria de módulos, dependem da classe de equivalência de Morita de $G$.

Prova-se que se a ação de $G$ em $M$ é localmente livre, então $G \ltimes M \rightrightarrows M$ é Morita equivalente a um grupoide étale, ver [7].

Com o exemplo apresentado acima, encontramos dois fatos que relacionam equivalência de Morita para Grupoides de Lie étale e equivalência de Morita para álgebras associativas:

- Se $G$ age em $M$ livremente e propriamente então $G \ltimes M \rightrightarrows M$ é Morita equivalente a um grupoide de Lie étale.

- Sob as mesmas hipóteses da ação de $G$ em $M$, as álgebras

$$
C_{c}^{\infty}(G \ltimes M \rightrightarrows M) \text { e } C_{c}^{\infty}(M / G)
$$

são Morita equivalentes.

Neste trabalho nos concentramos no caso em que os grupoides de Lie são étale. Temos o seguinte resultado, se dois grupoides de Lie étale são Morita equivalentes então suas respectivas álgebras de convolução são Morita equivalentes. É natural perguntarmos se existe alguma subcategoria desta categoria que é preservada por esta equivalência. Para respondermos esta pergunta, precisamos do seguinte resultado.

(Teorema de Serre-Swan para grupoides de Lie étale) Seja $G$ um grupoide de Lie étale sobre uma variedade $M$ diferenciável. Considere as categorias $\operatorname{Rep}(G)$ e $\operatorname{Mod}(G)$ das representações de $G$ e dos $C_{c}^{\infty}(G)$ - módulos de tipo finito e posto constante k. O funtor

$$
\begin{aligned}
\Gamma_{c}^{\infty}: \operatorname{Rep}(G) & \longrightarrow \operatorname{Mod}(G) \\
E \longmapsto & \longrightarrow \Gamma_{c}^{\infty}(E)
\end{aligned}
$$

é uma equivalência de categorias. 
Usando a equivalência de categorias dada pela extensão do Teorema de Serre-Swan para grupoides de Lie étale, obtemos que os módulos de tipo finito e posto constante são equivalentes. Para mostrarmos estes resultados, este trabalho está dividido da seguinte maneira.

No Capítulo 1, revisamos os conceitos básicos de fibrados vetoriais, módulos projetivos, livres e finitamente gerados sobre a álgebra de funções de uma variedade diferenciável e demonstramos o Teorema de Serre-Swan para variedades diferenciáveis, que estabelece uma equivalência entre a categoria dos fibrados vetoriais sobre uma variedade diferenciável $M$, denotada por $\operatorname{Vect}(M)$ e a categoria dos $C_{c}^{\infty}(M)$-módulos projetivos e finitamente gerados, denotada por $\operatorname{Mod}_{p f g}(M)$.

No Capítulo 2, apresentamos as definições de grupoide de Lie, representação de um grupoide de Lie e damos exemplos destas estruturas. Introduzimos o conceito de equivalência de Morita para grupoides de Lie, que é fundamental para este trabalho e mostramos que se dois grupoides de Lie são Morita equivalentes então a categoria das representações destes grupoides são equivalentes.

No Capítulo 3, nosso interesse está direcionado para grupoides de Lie étale, que são grupoides de Lie cujas funções estruturais são difeomorfismos locais. Definimos a álgebra de convolução de um grupoide de Lie $G \rightrightarrows M$, denotada por $C_{c}^{\infty}(G)$ e damos exemplos desta estrutura. Com este conceito, mostramos que o espaço das seções de toda representação de um grupoide de Lie possui uma estrutura natural de módulo sobre a álgebra de convolução deste grupoide. Em seguida, apresentamos os módulos de tipo finito e posto constante sobre tais álgebras e demonstramos o Teorema de Serre-Swan para grupoides de Lie étale, que estabelece uma equivalência entre a categoria das representações do grupoide de Lie, denotada por $\operatorname{Rep}(G)$ e a categoria dos $C_{c}^{\infty}(G)$-módulos de tipo finito e posto constante, denotada por $\operatorname{Mod}(\mathrm{G})$, estendendo o Teorema de Serre-Swan para variedades diferenciáveis.

No Capítulo 4, utilizamos os conceitos e resultados apresentados nos capítulos anteriores e provamos que, se dois grupoides de Lie étale são Morita equivalentes então suas álgebras de convolução são Morita equivalentes. Além disso, esta equivalência preserva a subcategoria de módulos de tipo finito e posto constante. 


\section{Capítulo 1}

\section{Teorema de Serre-Swan}

Neste capítulo estudaremos o Teorema de Serre-Swan para variedades diferenciáveis, o qual estabelece uma equivalência entre a categoria de fibrados vetoriais sobre uma variedade diferenciável $M$ e $C_{c}^{\infty}(M)$-módulos projetivos e finitamente gerados. A versão topológica do teorema foi demonstrada por Swan em [12].

\subsection{Fibrados vetoriais}

Nesta seção revisaremos brevemente o conceito de fibrado vetorial. Em seguida, apresentaremos alguns exemplos.

Definição 1.1.1. Um fibrado vetorial de posto $\mathbf{k}$ consiste em um espaço total $E$, uma base $M$, onde $E$ e $M$ são variedades diferenciáveis e uma submersão sobrejetora $\pi_{E}: E \rightarrow M$, chamada projeção, satisfazendo as seguintes condições.

i) Para todo $x \in M$, a fibra sobre $E_{x}:=\pi_{E}^{-1}(x)$ possui uma estrutura de $\mathbb{K}$-espaço vetorial de dimensão $k$;

ii) O espaço total E é localmente trivial, ou seja, para todo $x \in M$, existem um aberto $U \subset M$ com $x \in U$ e um difeomorfismo $\varphi: \pi_{E}^{-1}(U) \rightarrow U \times \mathbb{K}^{n}$ tal que $\operatorname{pr}_{1} \circ \varphi=\pi$ e a restrição $\varphi_{x}: E_{x} \rightarrow \mathbb{K}^{n}$ é um isomorfimo linear para todo $x \in U$, onde $\operatorname{pr}_{1}: U \times \mathbb{K}^{n} \rightarrow U$ é a projeção canônica sobre $U$.

Neste trabalho, fibrados vetoriais serão denotados pela função projeção $\pi_{E}: E \rightarrow M$ ou simplesmente diremos que $E$ é um fibrado vetorial sobre $M$. Consideramos $\mathbb{K}$ sendo um corpo de característica zero, mais frequentemente, $\mathbb{R}$ ou $\mathbb{C}$.

Exemplo 1.1.1. Sejam $M$ uma variedade diferenciável e $V$ um $\mathbb{K}$-espaço vetorial de dimensão $k$. A projeção $\mathrm{pr}_{1}: M \times V \rightarrow M$ define um fibrado vetorial cujas fibras se identificam com $V$. Este fibrado vetorial é chamado de fibrado trivial de posto $k$.

Exemplo 1.1.2. Seja $M$ uma variedade diferenciável. $O$ fibrado tangente $\pi_{M}: T M \rightarrow M$ é $O$ fibrado vetorial cuja fibra sobre cada ponto $p \in M$ é o espaço tangente $T_{p} M$.

Exemplo 1.1.3. Para todo fibrado vetorial $\pi_{E}: E \rightarrow M$, pode-se definir o fibrado dual $\pi_{E^{*}}: E^{*} \rightarrow$ $M$ cujas fibras são os espaços duais das fibras de E. Um caso importante de fibrado dual é o fibrado cotangente $\pi_{M^{*}}: T^{*} M \rightarrow M$, dual do fibrado tangente sobre $M$. 
Definição 1.1.2. Seja $\pi_{E}: E \rightarrow M$ um fibrado vetorial. Uma seção suave de E é uma aplicação $\sigma: M \rightarrow E$ tal que $\pi \circ \sigma=I d_{M}$. Denotaremos por $\Gamma^{\infty}(E)$ o conjunto das seções suaves de $E$ e por $\Gamma_{c}^{\infty}(E)$ o conjunto das seçôes suaves de $E$ com suporte compacto.

Dizemos que $\sigma_{1}, . ., \sigma_{k} \in \Gamma^{\infty}(E)$ são linearmente independentes se $\sigma_{1}(p), . ., \sigma_{k}(p) \in E_{p}$ são linearmente independentes para cada $p \in M$.

No caso do fibrado tangente, as seções do fibrado $T M$ sobre uma variedade diferenciável $M$ são os campos de vetores tangentes a $M$.

Por outro lado, as seções do fibrado cotangente de uma variedade diferenciável $M$ são as 1-formas diferenciais em $M$.

Definição 1.1.3. Sejam $\pi_{E}: E \rightarrow M$ um fibrado vetorial e $U \subset M$ um aberto de $M$. Uma seção local em $U$ é uma aplicação suave $\sigma: U \rightarrow E$ tal que $\pi_{E} \circ \sigma=I d_{U}$.

Se $\pi_{E}: E \rightarrow M$ é um fibrado vetorial e $U \subset M$ é aberto de $M$, então $\left.\pi_{E}\right|_{W}: \pi_{E}^{-1}(U) \rightarrow U$ define um fibrado vetorial sobre o aberto $U$. Este novo fibrado será denotado por $\left.E\right|_{U}$ e diremos que ele é o fibrado restrito ao aberto $U$. Desta forma, o espaço das seções locais de $E$ pode ser identificado com o espaço das seções do fibrado restrito $\left.E\right|_{U}$, ou seja, o espaço das seções locais é $\Gamma^{\infty}\left(\left.E\right|_{U}\right)$.

Exemplo 1.1.4. Considere o fibrado tangente $\pi_{M}: T M \rightarrow M$ e considere uma carta local definida num aberto $U$ de $M$ com coordenadas $\left(x_{1}, \ldots, x_{k}\right)$. Vamos definir um difeomorfismo

$$
\begin{aligned}
U \times \mathbb{K}^{k} & \left.\longrightarrow(T M)\right|_{U}=\pi_{M}^{-1}(U) \\
\left(p, \lambda_{1}, \ldots, \lambda_{k}\right) & \left.\longmapsto \sum_{i=1}^{k} \lambda_{i} \frac{\partial}{\partial x_{i}}\right|_{p},
\end{aligned}
$$

onde $\left.\frac{\partial}{\partial x_{i}}\right|_{p} \in T_{p} M$ denota o vetor tangente correspondente ao operador de derivação no ponto $p$ em relação à coordenada $x_{i}$. Para cada $p \in U$ os vetores $\left\{\left.\frac{\partial}{\partial x_{i}}\right|_{p}\right\}_{1 \leqslant i \leqslant k}$ formam uma base de $T_{p} M$. Portanto, as seçôes $\frac{\partial}{\partial x_{i}}: U \rightarrow T M$ são seções locais linearmente independentes. Para cada $p \in U$ considere a base $\left\{d x_{1}(p), \ldots, d x_{k}(p)\right\}$ de $T_{p}^{*} M$ dual da base $\left\{\left.\frac{\partial}{\partial x_{i}}\right|_{p}\right\}_{1 \leqslant i \leqslant k}$. Da mesma forma obtemos seçôes locais

$$
d x_{i}: U \longrightarrow T^{*} M \quad \text { para } 1 \leqslant i \leqslant k
$$

linearmente independentes.

\subsection{Morfismos de fibrados vetoriais}

Dados dois fibrados vetoriais $\pi_{E_{1}}: E_{1} \rightarrow M_{1}$ e $\pi_{E_{2}}: E_{2} \rightarrow M_{2}$, um morfismo entre eles consiste de duas funções $\phi: E_{1} \rightarrow E_{2}$ e $f: M_{1} \rightarrow M_{2}$ tais que o seguinte diagrama é comutativo

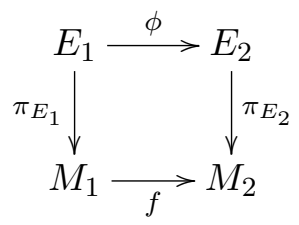


e a função $\phi$, quando restrita a cada fibra $\pi_{E_{1}}^{-1}(p)$, define uma transformação linear

$$
\left.\phi\right|_{\pi_{E_{1}}^{-1}(p)}: \pi_{E_{1}}^{-1}(p) \rightarrow \pi_{E_{2}}^{-1}(f(p)) .
$$

Neste trabalho, consideraremos morfismos entre fibrados sobre a mesma base $M$ onde $f$ é a função identidade. Neste caso, teremos um diagrama comutativo mais simples,

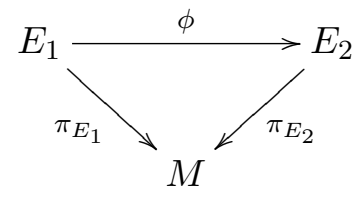

sendo que para todo $p \in M$ a função $\phi_{p}:=\left.\phi\right|_{\pi_{E_{1}}^{-1}(p)}: \pi_{E_{1}}^{-1}(p) \rightarrow \pi_{E_{2}}^{-1}(p)$ é uma transformação linear entre as fibras sobre o mesmo ponto.

Diremos que $\phi$ é um morfismo de fibrados vetoriais que cobre a identidade ou simplesmente, $\phi$ é um morfismo de fibrados vetoriais. Um isomorfismo de fibrados vetoriais é um morfismo de fibrados vetoriais bijetor. De forma equivalente, $\phi_{p}: \pi_{E_{1}}^{-1}(p) \rightarrow \pi_{E_{2}}^{-1}(p)$ é um isomorfismo linear. E denotaremos $E_{1} \simeq E_{2}$.

Uma vez estabelecido o conceito de isomorfismo entre fibrados, podemos dar a definição de fibrado trivializável. Dizemos que um fibrado $E$ sobre $M$ de posto $k$ é trivializável quando $E \simeq$ $M \times \mathbb{K}^{k}$, ou seja, quando $E$ é isomorfo ao fibrado $M \times \mathbb{K}^{k}$. Segue da definição que, todo fibrado vetorial $E$ é localmente trivial, ou seja, sempre existe uma vizinhança $U \subset M$ tal que $\left.E\right|_{U}$ é um fibrado trivial. Uma tal vizinhança $U \subset M$ onde $E$ é trivial, será chamada de vizinhança trivializante de $E$.

O próximo teorema é uma consequência natural da definição de fibrado vetorial e do conceito de isomorfismo de fibrados.

Teorema 1.2.1. Seja $E \rightarrow M$ um fibrado vetorial de posto $k$. Então $E$ é trivializável se, e somente se, existem $k$ seçôes $\sigma_{1}, \ldots, \sigma_{k} \in \Gamma^{\infty}(E)$ linearmente independentes.

Demonstração. Basta lembrarmos que ser trivializável é por definição existir um isomorfismo de fibrados

$$
\phi: M \times \mathbb{K}^{k} \rightarrow E
$$

Para construir as seções $\sigma_{i}: M \rightarrow E$, basta fazermos $\sigma_{i}(x)=\phi\left(x, e_{i}\right)$, para todo $x \in M$ onde $\left\{e_{i}\right\}_{1 \leqslant i \leqslant k}$ é a base canônica de $\mathbb{K}^{k}$.

Sejam $\pi_{E}: E \rightarrow M$ um fibrado vetorial de posto $k$ e $U \subset M$ um aberto trivializante de $E$. Os isomorfismos dados por trivializações locais da forma

$$
\left.E\right|_{U}=\pi_{E}^{-1}(U) \rightarrow U \times \mathbb{K}^{k}
$$

também serão chamados de cartas fibradas. Tomando a função inversa de uma carta fibrada, podemos facilmente definir $k$ seções locais

$$
\sigma_{1}, \ldots, \sigma_{k} \in \Gamma^{\infty}\left(\left.E\right|_{U}\right)
$$


linearmente independentes. Em particular, para qualquer $\sigma \in \Gamma^{\infty}(E)$ temos

$$
\sigma(x)=\sum_{i=1}^{k} a_{i}(x) \sigma_{k}(x), \text { para todo } x \in U, \text { onde } a_{1}, \ldots, a_{k} \in C^{\infty}(U) \text {. }
$$

Observação 1.2.1. Com o que vimos acima, podemos concluir que dar uma trivialização local é equivalente a dar uma base de seçôes locais.

A seguir provaremos uma proposição útil para a demonstração do Teorema de Serre-Swan.

Proposição 1.2.1. Seja $\pi_{E}: E \rightarrow M$ um fibrado vetorial. Para todo ponto $p \in M$ e toda seção local $\sigma: U \rightarrow E$ de $E$ definida numa vizinhança aberta $U \subset M$ do ponto $p$, existe uma seção $\bar{\sigma}: M \rightarrow E$, tal que $\sigma$ e $\bar{\sigma}$ coincidem em uma vizinhança $W \subset U$ de $p$.

Demonstração. Seja $p \in U \subset M$ e $e \in \pi_{E}^{-1}(p)$. Então, tomando uma trivialização local de $E$, sabemos que existe $\sigma \in \Gamma^{\infty}\left(\left.E\right|_{U}\right)$ tal que $\sigma(p)=e$. Como $M$ é uma variedade diferenciável, podemos considerar duas vizinhanças abertas $V$ e $W$ de $p$ tais que $\bar{W} \subset V \subset \bar{V} \subset U$.

Dadas tais vizinhanças, sabemos que existe uma função $h: M \rightarrow \mathbb{K}$ tal que $\left.h\right|_{\bar{W}} \equiv 1$ e $\left.h\right|_{M \backslash V} \equiv 0$. Assim, definimos $\bar{\sigma}: M \rightarrow E$ por

$$
\bar{\sigma}(y)= \begin{cases}h(y) \sigma(y), & \text { se } y \in U \\ 0, & \text { caso contrário. }\end{cases}
$$

Como $h$ se anula fora do aberto $V$, temos que $\bar{\sigma}$ é diferenciável de classe $C^{\infty}$. Como $h$ é constante igual a 1 sobre $\bar{W}$, temos que $\bar{\sigma}$ é uma seção global que coincide com $\sigma$ em $W$, conforme queríamos demonstrar.

Definição 1.2.1. Sejam $\pi_{E}: E \rightarrow M$ um fibrado vetorial. Um subfibrado de $E$ é um fibrado vetorial $\pi_{E^{\prime}}: E^{\prime} \rightarrow M^{\prime}$, onde $E^{\prime} \subset E$ e $M^{\prime} \subset M$ são subvariedades e $E_{x^{\prime}}^{\prime} \subset E_{x^{\prime}}$ é um subespaço vetorial de para todo $x^{\prime} \in E^{\prime}$, ou seja, a inclusão define um morfismo de fibrados,

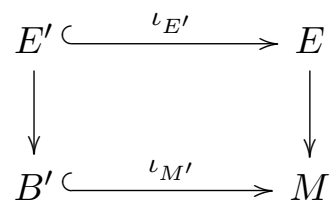

Definição 1.2.2. Seja $\phi: E \rightarrow F$ um morfismo de fibrados vetoriais diferenciáveis que cobre a identidade. Definimos Ker $\phi$ e $\operatorname{Im} \phi$ como os conjuntos de todos os núcleos e imagens de $\phi$ por:

$$
\begin{aligned}
\operatorname{Ker} \phi & =\bigcup_{x \in M} \operatorname{Ker}\left(\phi_{x}\right) \\
\operatorname{Im} \phi & =\bigcup_{x \in M} \operatorname{Im}\left(\phi_{x}\right) .
\end{aligned}
$$

O exemplo a seguir nos mostra que o $\operatorname{Ker} \phi$ e $\operatorname{Im} \phi$ não são subfibrados vetorias de $E$ e $F$, respectivamente.

Exemplo 1.2.1. Considere $I=[0,1], E=I \times \mathbb{R}$ é o fibrado vetorial trivial $\pi_{E}: E \rightarrow I$. A aplicação $\phi: E \rightarrow E$ dada por $(x, y)=(x, x y)$ é um morfismo de fibrados vetoriais. 
Se $x=0$ então $\operatorname{Ker}\left(\phi_{x}\right)=\{0\} \times \mathbb{R}$. Logo, $\operatorname{dim}\left(\operatorname{Ker}\left(\phi_{0}\right)\right)=1$. Além disso, $\operatorname{dim}\left(\operatorname{Im}\left(\phi_{0}\right)\right)=0$.

Se $x \neq 0$ então $\operatorname{Ker}\left(\phi_{x}\right)=\{0\} \times\{0\}$. Logo, $\operatorname{dim}\left(\operatorname{Ker}\left(\phi_{x}\right)\right)=0$. Além disso, $\operatorname{dim}\left(\operatorname{Im}\left(\phi_{x}\right)=1\right.$.

Considere $\phi: E \rightarrow F$ é um morfismo de vibrados vetoriais que cobre a identidade, o teorema a seguir nos fornece condições para que $\operatorname{Ker} \phi$ e $\operatorname{Im} \phi$ sejam subfibrados vetoriais de $E$ e $F$, respectivamente.

Proposição 1.2.2. Se $\phi: E \rightarrow F$ é um morfismo de fibrados vetoriais com posto constante então $\operatorname{Ker} \phi \subset E$ e $\operatorname{Im} \phi \subset F$ são subfibrados vetoriais.

Demonstração. Ver [12].

Observação 1.2.2. Dado $\phi: E \rightarrow F$ um morfismo de fibrados vetoriais diferenciáveis que cobre a identidade. Temos que a dimensão de $\operatorname{Im} \phi$ em torno de um ponto não descresce. Analogamente, a dimensão de Ker $\phi$ em torno de um ponto não cresce. De fato, para cada ponto $x \in M, \operatorname{dim}\left(\operatorname{Im}\left(\phi_{x}\right)\right)$ é o número de colunas linearmente independentes em qualquer matriz de $\phi_{x}$. Se uma tal matriz de $\phi_{x}$ possuir um total de $k$ colunas linearmente independentes, então $\operatorname{dim}\left(\operatorname{Im}\left(\phi_{x}\right)\right)=k$. Assim sendo, podemos escolher entradas dessas colunas e construir uma matriz $k \times k$ invertivel que denotaremos por $A_{k}$. Dessa forma, $\operatorname{det}\left(A_{k}\right) \neq 0$. Como $\operatorname{dim}(\operatorname{Im} \phi)+\operatorname{dim}(\operatorname{Ker} \phi)$ é constante, segue que se a $\operatorname{dim}(\operatorname{Im} \phi)$ não decresce, consequentemente a $\operatorname{dim}(\operatorname{Ker} \phi)$ não cresce.

\subsection{Módulos projetivos e módulos finitamente gerados}

Seja $M$ uma variedade diferenciável. Denotaremos por $C_{c}^{\infty}(M)$ o conjunto das funções suaves de $M$ em $\mathbb{K}$ com suporte compacto.

Para todo fibrado vetorial $E$ sobre $M$, o espaço vetorial $\Gamma_{c}^{\infty}(E)$ das seções suaves de $E$ com suporte compacto admite uma estrutura natural de $C_{c}^{\infty}(M)$-módulo dada por

$$
(f \sigma)(x):=f(x) \sigma(x), \quad x \in M
$$

onde $f \in C_{c}^{\infty}(M)$ e $\sigma \in \Gamma_{c}^{\infty}(E)$. Todo morfismo $\phi: E \rightarrow F$ de fibrados vetoriais que cobre a identidade induz um morfismo de $C_{c}^{\infty}(M)$-módulos

$$
\begin{aligned}
\Gamma_{c}^{\infty}(\phi): \Gamma_{c}^{\infty}(E) & \longrightarrow \Gamma_{c}^{\infty}(F) \\
\sigma & \longmapsto \phi \circ \sigma .
\end{aligned}
$$

Proposição 1.3.1. Seja $I_{x}$ o ideal em $C^{\infty}(M)$ das funçôes que se anulam em $x$. Entẫo $\Gamma_{c}^{\infty}(E) / I_{x} \Gamma_{c}^{\infty}(E)$ é isomorfo como espaço vetorial a $E_{x}$.

Demonstração. Dado $x \in M$, definimos $f_{x}: \Gamma_{c}^{\infty}(E) \rightarrow E_{x}$ dada por $f_{x}(\sigma)=\sigma(x)$. A função $f_{x}$ é um morfismo de espaços vetoriais. Seja $\sigma \in \operatorname{Ker} f_{x}$, temos

$$
f_{x}(\sigma)=0 \Longrightarrow \sigma(x)=0 \Longrightarrow \sigma \in I_{x} \Gamma_{c}^{\infty}(E)
$$

Logo, $\operatorname{Ker} f_{x} \subset I_{x} \Gamma_{c}^{\infty}(E)$. Por outro lado, se $\sigma \in I_{x} \Gamma_{c}^{\infty}(E)$, então

$$
\sigma(x)=0 \Longrightarrow f_{x}(\sigma)=0 \Longrightarrow \sigma \in \operatorname{Ker} f_{x} .
$$


Logo, $I_{x} \Gamma_{c}^{\infty}(E) \subset \operatorname{Ker} f_{x}$. Segue que, $\operatorname{Ker} f_{x}=I_{x} \Gamma_{c}^{\infty}(E)$. Seja $e \in E_{x}$, pela observação 1.2.1, existe $\sigma \in \Gamma_{c}^{\infty}(E)$ tal que $\sigma(x)=e$. Logo, $f_{x}(\sigma)=e$. Portanto, $f_{x}$ é sobrejetora. Pelo Teorema do isomorfismo, temos

$$
\Gamma_{c}^{\infty}(E) / I_{x} \Gamma_{c}^{\infty}(E) \simeq E_{x}
$$

Agora revisaremos os conceitos de módulo finitamente gerado, livre e projetivo.

Definição 1.3.1. Seja $P$ um módulo sobre um anel comutativo $A$.

i) $O$ módulo $P$ é dito finitamente gerado quando existir um conjunto finito $\left\{e_{i} \in P ; i=1, \ldots, n\right\}$ tal que todo elemento $p \in P$ pode ser escrito na forma

$$
p=a_{1} e_{1}+\cdots+a_{n} e_{n},
$$

com $a_{1}, \ldots, a_{n} \in A$.

ii) Dizemos que o módulo $P$ é livre quando for isomorfo a uma soma direta de cópias do anel A. Isto é:

$$
P \simeq \bigoplus_{\lambda \in \Lambda} A
$$

iii) O módulo $P$ é dito projetivo se for somando direto de um módulo livre. Ou seja, se existe um módulo $S$ tal que $P \oplus S$ é um módulo livre.

Observamos que na condição i) da definição acima os elementos de $P$ podem ser escritos como combinações lineares (1.12) de forma não necessariamente única. Já na condição ii), o isomorfismo (1.13) corresponde à existência de uma base para o módulo. Assim sendo, caso todos elementos do módulo possam ser escritos de forma única como combinação linear dos geradores, então o módulo tem uma base que corresponde a um isomorfismo do tipo (1.13). Isto é, o módulo é livre. Ou seja, módulos livres são aqueles que admitem uma base.

Proposição 1.3.2. Considere $\pi_{E}: E \longrightarrow M$ um fibrado vetorial. Suponha que $\operatorname{dim} E_{x}=n$, para todo $x \in M, \operatorname{dim} M=m$, e que $M$ admite um atlas de cartas adaptas de no máximo $(m+1)$ trivializações locais. $O C_{c}^{\infty}(M)$-módulo $\Gamma_{c}^{\infty}(E)$ é projetivo e finitamente gerado.

Demonstração. Para cada $x \in M$, podemos escolher $\sigma_{1}, \ldots, \sigma_{n}$ tais que $\left\{\sigma_{i}(x)\right\}$ é uma base em torno de $x$. Os abertos relativos a tais bases definem um atlas de trivializações para $E$, dado por

$$
\begin{aligned}
\pi_{E}^{-1}(U) & \longrightarrow R^{n+m} \\
e & \longmapsto\left(a_{1}(x), \ldots, a_{n}(x), \varphi(x)\right),
\end{aligned}
$$

onde $(\varphi, U)$ é carta local de $M, x \in U, e \in E_{x}$ tal que $e=\sum_{i=1}^{n} a_{i}(x) \sigma_{i}(x)$ com $a_{i}: M \longrightarrow \mathbb{R}$ são funções suaves.

Assim, para cada $x \in M$, obtemos um atlas de trivializações para $E$. Como $M$ admite um atlas de cartas adaptas de no máximo $(m+1)$ trivializações locais, para cada trivialização, temos $n$ seções locais. Logo, existe um número finito $N=n(m+1)$ de seções em $\Gamma_{c}^{\infty}(E)$ tais que $\left\{\sigma_{1}, \ldots, \sigma_{n}, \ldots, \sigma_{N}\right\}$ que geram $E_{x}$ para todo $x \in M$. 
Considere $\left\{\phi_{i}\right\}_{\{i \in I\}}$ uma partição da unidade subordinhada a cobertura $\left\{U_{i}\right\}$ de $M$. Temos que cada $\phi_{i}$ é positiva, tem suporte compacto $U_{i}$ e $\sum_{i=1}^{n} \phi_{i}=1$.

Dada $\sigma \in \Gamma_{c}^{\infty}(E)$. Para cada aberto trivializante $U_{i}$, temos $n$ seções que geram as fibras localmente. Ou seja, $\sigma=a_{1} \sigma_{1_{i}}+\cdots+a_{n_{i}} \sigma_{n_{i}}$. Agora, observamos que, como para cada $U_{i}$ temos $\sum_{i=1}^{n} \phi_{i}=1$, então

$$
\begin{gathered}
a_{1} \phi_{1} \sigma_{1_{1}}+\cdots+a_{n_{1}} \phi_{1} \sigma_{n_{1}}+a_{n_{2}} \phi_{2} \sigma_{1_{2}}+\cdots+a_{n_{2}} \phi_{2} \sigma_{n_{2}}+\ldots \\
\ldots+a_{n_{N}} \phi_{N} \sigma_{1_{N}}+\cdots+a_{n_{N}} \phi_{N} \sigma_{n_{N}}=\sigma .
\end{gathered}
$$

Isto prova que $\Gamma_{c}^{\infty}(E)$ é finitamente gerado.

Mostraremos que $\Gamma_{c}^{\infty}(E)$ é projetivo. Ou seja, $\Gamma_{c}^{\infty}(E)$ é somando direto de um livre. Considere o fibrado trivial $M \times \mathbb{R}^{N}$, temos que $\Gamma_{c}^{\infty}(F)$ é $C_{c}^{\infty}(M)$-módulo livre sendo $\alpha_{1}, \ldots, \alpha_{N}$ seus gerados. Definimos

$$
\begin{gathered}
\Phi: \Gamma_{c}^{\infty}(F) \longrightarrow \Gamma_{c}^{\infty}(E) \\
\alpha_{i} \longmapsto \sigma_{i}
\end{gathered}
$$

Como o funtor $\Gamma_{c}^{\infty}$ é pleno, fato mostrado no final deste capítulo, existe $\phi: F \longrightarrow E$ tal que $\Gamma_{c}^{\infty}(\phi)=\Phi$. Então

$$
\Phi\left(\alpha_{i}\right)=\sigma_{i} \Longrightarrow \sigma(x) \in \operatorname{Im} \phi, \text { para todo } x \in M \text {. }
$$

Como $\left\{\sigma_{i}(x)\right\}$ geram a fibra $E_{x}$ para cada $x \in M$, obtemos que $\phi$ é morfismo sobrejetor. Logo, $\operatorname{Im} \phi$ é constante, pela Proposição 1.2.2, temos que $D=\operatorname{Ker} \phi$ é um subfibrado vetorial de $F$. Assim, existe $D^{\prime}$ um subfibrado vetorial de $F$ tal que $F=D^{\prime} \oplus D$. Como $\phi$ é um morfismo sobrejetor onde $D=\operatorname{Ker} \phi$, temos que $D^{\prime} \simeq E$. Ou seja, $F \simeq D \oplus E$. Pelo fato de $\Gamma_{c}^{\infty}$ ser aditivo, segue que,

$$
\Gamma_{c}^{\infty}(F)=\Gamma_{c}^{\infty}(D \oplus E)=\Gamma_{c}^{\infty}(D) \oplus \Gamma_{c}^{\infty}(E) .
$$

Portanto, existe $\Gamma_{c}^{\infty}(D)$ tal que $\Gamma_{c}^{\infty}(D) \oplus \Gamma_{c}^{\infty}(E)$ é $C_{c}^{\infty}(M)$-módulo livre. O que conclui a demonstração.

\subsection{Teorema de Serre-Swan}

Nas seções anteriores, reunimos os conceitos necessários para demonstrarmos a versão diferenciável do Teorema de Serre-Swan. Esta seção é basicamente dedicada à sua demonstração.

Denotaremos por $\operatorname{Vect}(M)$ a categoria dos fibrados vetoriais sobre uma base fixada $M$ e por $\operatorname{Mod}_{p f g}(M)$ a categoria dos $C_{c}^{\infty}(M)$-módulos projetivos e finitamente gerados.

Seja $E$ um fibrado vetorial sobre $M$, pelo exemplo 1.3.2 temos que $\Gamma_{c}^{\infty}(E)$ é um $C_{c}^{\infty}(M)$-módulo projetivo e finitamente gerado.

Se $\phi$ é um morfismo de fibrados vetoriais que cobre a identidade, então $\Gamma_{c}^{\infty}(\phi)$ é uma aplicação $C_{c}^{\infty}(M)$-linear. Em particular, $\Gamma_{c}^{\infty}(\phi)\left(I d_{E}\right)=I d_{E}$. Considere $\psi: E_{1} \rightarrow E_{2}$ e $\phi: E_{2} \rightarrow E_{3}$ morfismos 
de fibrados vetoriais, para toda $\sigma \in \Gamma_{c}^{\infty}(E)$ temos,

$$
\Gamma_{c}^{\infty}(\phi \circ \psi)(\sigma)=(\phi \circ \psi)(\sigma)=\phi\left(\Gamma_{c}^{\infty}(\psi)(\sigma)\right)=\left(\Gamma_{c}^{\infty}(\phi) \circ \Gamma_{c}^{\infty}(\psi)\right)(\sigma) .
$$

Portanto, $\Gamma_{c}^{\infty}$ é um funtor entre as categorias $\operatorname{Vect}(M)$ e $\operatorname{Mod}_{p f g}(M)$. Além disso, a aplicação

$$
\begin{aligned}
\operatorname{Hom}_{\mathrm{Vect}(M)}(E, F) & \longrightarrow \operatorname{Hom}_{C_{c}^{\infty}-M o d}\left(\Gamma_{c}^{\infty}(E), \Gamma_{c}^{\infty}(F)\right) \\
\phi & \longmapsto \Gamma_{c}^{\infty}(\phi)
\end{aligned}
$$

é um morfismo de grupos abelianos.

Teorema 1.4.1. (Serre-Swan) Seja M uma variedade diferenciável. O funtor

$$
\begin{aligned}
\Gamma_{c}^{\infty}: \operatorname{Vect}(M) & \longrightarrow \operatorname{Mod}_{p f g}(M) \\
E \longmapsto & \longrightarrow \Gamma_{c}^{\infty}(E)
\end{aligned}
$$

é uma equivalência de categorias.

Demonstração. Mostraremos que $\Gamma_{c}^{\infty}$ é um funtor fiel, pleno e denso. Vamos começar provando que tal funtor é fiel. Ou seja, dados $\phi, \psi: E \rightarrow F$ morfismos de fibrados vetoriais tais que $\Gamma_{c}^{\infty}(\phi)=$ $\Gamma_{c}^{\infty}(\psi)$, mostraremos que $\phi=\psi$. Sejam $x \in U \subset M$ e $e \in E_{x}$. Ou seja, $\pi_{E}(e)=x$ e $\sigma \in \Gamma_{c}^{\infty}(E)$ tal que $\sigma(x)=e$. Pela Proposição 1.2.1, existe $\bar{\sigma} \in \Gamma_{c}^{\infty}(E)$, tal que $\bar{\sigma}(x)=e$. Assim,

$$
\phi(e)=\phi(\bar{\sigma}(x))=\left(\Gamma_{c}^{\infty}(\phi)\right)(\bar{\sigma})(x)=\left(\Gamma_{c}^{\infty}(\psi)\right)(\bar{\sigma})(x)=\psi \circ \bar{\sigma}(x)=\psi(e) .
$$

Portanto, $\phi=\psi$ para todo $e \in E$. Segue que, $\Gamma_{c}^{\infty}$ é fiel.

Agora mostraremos que o funtor $\Gamma_{c}^{\infty}$ é pleno. Ou seja, para todo $\Phi: \Gamma_{c}^{\infty}(E) \rightarrow \Gamma_{c}^{\infty}(F)$ um morfismo de $C_{c}^{\infty}(M)$-módulos, existe $\phi: E \rightarrow F$ morfismo de fibrados vetoriais tal que $\Phi=\Gamma_{c}^{\infty}(\phi)$. Fixado $x \in M$. Considere $I_{x} \Gamma_{c}^{\infty}(E)$ o espaço das seções que se anulam em $x$ e o isomorfismo $\Gamma_{c}^{\infty}(E) / I_{x} \Gamma_{c}^{\infty}(E) \simeq E_{x}$ apresentado na Proposição 1.3.1. Assim, basta sabermos o valor das seções em $x$.

Seja $\Phi: \Gamma_{c}^{\infty}(E) \rightarrow \Gamma_{c}^{\infty}(F)$ um morfismo de $C_{c}^{\infty}$-módulos, definimos

$$
\begin{gathered}
\bar{\Phi}:=\phi_{x}: \Gamma_{c}^{\infty}(E) / I_{x} \Gamma_{c}^{\infty}(E) \longrightarrow \Gamma_{c}^{\infty}(F) / I_{x} \Gamma_{c}^{\infty}(F) \\
{[\sigma] \longmapsto[\Phi(\sigma)]}
\end{gathered}
$$

Desta forma, obtemos uma família de funções lineares que induzem uma aplicação $\phi$ entre os fibrados vetoriais $E$ e $F$ que é linear fibra a fibra.

Sejam $\sigma_{1}, \ldots, \sigma_{n} \in \Gamma_{c}^{\infty}(E)$ tais que $\sigma_{1}(x), \ldots, \sigma_{n}(x)$ formam uma base em $E_{x}$. Dado $e \in E_{x}$, temos

$$
e=\sum_{i=1}^{n} a_{i}(x) \sigma_{i}(x), \text { onde } a_{i} \in C_{c}^{\infty}(M), \text { para todo } i .
$$

Logo,

$$
\phi(e)=\sum_{i=1}^{n} a_{i}(x) \phi \circ \sigma_{i}(x) .
$$


Para cada $i$ e para cada $x \in M$,

$$
\left(\phi \circ \sigma_{i}\right)(x)=\phi_{x}\left(\sigma_{i}(x)\right)=\left(\bar{\Phi}\left(\sigma_{i}\right)\right)(x)=\left(\Phi\left(\left[\sigma_{i}\right]\right)\right)(x) .
$$

Como $\Phi$ leva seções suaves em seções suaves temos que $\phi$ é suave em cada fibra. Portanto, $\phi$ é suave em $E$. Além disso, $\phi$ é morfismo de fibrados vetoriais. De fato,

$$
\pi_{F} \circ \phi(e)=\pi_{F} \circ \phi\left(\sigma_{i}(x)\right)=\left(\pi_{F} \circ\left(\bar{\Phi}\left(\sigma_{i}(x)\right)\right)\right)(x)=\left(\pi_{E} \circ \sigma_{i}\right)(x)=x .
$$

Dado $\sigma \in \Gamma_{c}^{\infty}(E)$, para cada $x \in M$, temos

$$
\left(\Gamma_{c}^{\infty}(\phi)(s)\right)(x)=\phi(\sigma(x))=\phi_{x}(\sigma(x))=(\phi(\sigma))(x) .
$$

Portanto, $\Phi=\Gamma_{c}^{\infty}(\phi)$. Segue que, $\Gamma_{c}^{\infty}$ é pleno.

Finalmente, provaremos que o funtor $\Gamma_{c}^{\infty}$ é denso. Ou seja, seja $P$ um $C_{c}^{\infty}(M)$-módulo projetivo e finitamente gerado, mostraremos que existe um fibrado vetorial $E$ sobre $M$ tal que $\Gamma_{c}^{\infty}(E) \simeq P$. Como $P$ é projetivo e finitamente gerado, existe um $C_{c}^{\infty}(M)$-módulo livre e finitamente gerado $Q$ tal que $Q \simeq P \oplus S$.

Seja $\psi: P \oplus S \rightarrow P \oplus S$ dada por $\psi(p+s)=p+0=p$, temos $\psi \in \operatorname{End}_{C_{c}^{\infty}(M)}(P \oplus S)$. Note que, $\psi^{2}=\psi$. De fato,

$$
\psi(p+s)=p \Rightarrow \psi(\psi(p+s))=\psi(p)=\psi(p+s), \forall(p+s) \in P \oplus S .
$$

Portanto, $\psi$ é uma projeção. Fazendo $\left.\psi\right|_{P}: P \rightarrow P \oplus S$, temos $\operatorname{Im} \psi \simeq P$.

Como $Q$ é livre, $Q$ pode ser associado a um fibrado trivial $F$ sobre $M$ da seguinte forma, $Q=\Gamma_{c}^{\infty}(F)$. Existe um único morfismo $\phi: F \rightarrow F$ que cobre a identidade tal que $\psi=\Gamma_{c}^{\infty}(\phi)$ pois o funtor $\Gamma_{c}^{\infty}$ é fiel e pleno. O morfismo $\phi$ é idempotente pois $\psi$ é projeção e $\Gamma_{c}^{\infty}$ é fiel.

Sejam $D=\operatorname{Ker} \phi$ e $E=\operatorname{Im} \phi$. Todo elemento $z$ de $F$ pode ser escrito como $z-\phi(z)+\phi(z)$, onde $z-\phi(z) \in \operatorname{Ker} \phi$ e $\phi(z) \in \operatorname{Im} \phi$. Logo, $F=\operatorname{Ker} \phi+\operatorname{Im} \phi=D+E$. Observe que, se $z \in \operatorname{Ker} \phi$ e $z \in \operatorname{Im} \phi$ então $\phi(z)=0$ e existe $w \in F$ tal que $\phi(w)=z$. Logo,

$$
\phi^{2}(w)=\phi(z)=0 \Rightarrow \phi^{2}(w)=0 \Rightarrow z=0 .
$$

Portanto, $\operatorname{Ker} \phi \cap \operatorname{Im} \phi=\{0\}$ e concluímos que, $F=\operatorname{Ker} \phi \oplus \operatorname{Im} \phi$.

Para cada $x \in M$. Considere $\operatorname{dim} F_{x}=q, \operatorname{dim} E_{x}=p$ e $\operatorname{dim} D_{x}=r$, pelo Teorema do núcleo e da imagem $r=q-p$. Pela Observação $1.2 .2, \operatorname{dimKer} \phi \leq r$. Como $\phi$ e $i d_{F}-\phi$ são idempotentes temos $\operatorname{Ker} \phi=\operatorname{Im}\left(i d_{F}-\phi\right)$ e $\operatorname{Im} \phi=\operatorname{Ker}\left(i d_{F}-\phi\right)$ e $F=\operatorname{Ker}\left(i d_{F}-\phi\right) \oplus \operatorname{Im}\left(i d_{F}-\phi\right)=\operatorname{Im} \phi \oplus \operatorname{Ker} \phi$. Então, $\operatorname{dim} \operatorname{Im}\left(i d_{F}-\phi\right) \geq r$ e $\operatorname{dimKer} \phi \geq r$. Portanto, $\operatorname{dimKer} \phi=r$. Pela Proposição 1.2.2, Ker $\phi$ e $\operatorname{Im} \phi$ são subfibrados de $F$ onde $F=D \oplus E$.

Pela aditividade de $\Gamma_{c}^{\infty}$ obtemos $\Gamma_{c}^{\infty}(F)=\Gamma_{c}^{\infty}(D) \oplus \Gamma_{c}^{\infty}(E)$. Pela Proposição 1.3.2, $\Gamma_{c}^{\infty}(E)$ é $C_{c}^{\infty}(M)$-módulo projetivo e finitamente gerado. Além disso, observamos que

$$
\Gamma_{c}^{\infty}(E)=\Gamma_{c}^{\infty}(\operatorname{Im} \phi)=\operatorname{Im}\left(\Gamma_{c}^{\infty}(\phi)\right)=\operatorname{Im} \psi \simeq P
$$

Portanto, dado $C_{c}^{\infty}(M)$-módulo projetivo e finitamente gerado $P$, existe um fibrado vetorial $E$ sobre $M$ tal que $P \simeq \Gamma_{c}^{\infty}(E)$. Segue que, $\Gamma_{c}^{\infty}$ é denso, o que conclui a demonstração. 


\section{Capítulo 2}

\section{Grupoides de Lie}

Neste capítulo introduzimos o conceito de grupoide de Lie. Também estudamos noções importantes relacionadas a grupoides de Lie, tais como: ações e representações de grupoides de Lie e equivalência de Morita. Para as três primeiras seções deste capítulo nossas principais referências são [6], [1], [5], [2] e para a última seção, na qual falaremos sobre equivalência de Morita para grupoides de Lie, seguimos [8] e [3].

\subsection{Grupoides: definições e exemplos.}

Definição 2.1.1. Um grupoide consiste em um conjunto de objetos $M$, um conjunto de flechas $G$ e aplicaçôes estruturais:

i) Duas aplicações $s, t: G \rightarrow M$ chamadas respectivamente de source $e$ target.

ii) Um produto $m$ definido parcialmente entre flechas componíveis $m: G{ }_{s} \times{ }_{t} G \rightarrow G$ dada por $m(g, h)=g h$, onde $G_{s} \times_{t} G=\{(g, h) \in G \times G ; s(g)=t(h)\}$. A multiplicação $m$ é associativa no seguinte sentido: $m(g h, k)=(g h) k=g(h k)=m(g, h k)$, sempre que as composições $g h,(g h) k, h k$ e $g(h k)$ estiverem definidas.

iii) Uma aplicação $\epsilon: M \rightarrow G$, dada por $\epsilon(x)=\epsilon_{x}$ tal que para toda flecha $g \in G$, com $s(g)=x$ e $t(g)=y, g \epsilon_{x}=g$ e $\epsilon_{y} g=g$. A aplicação $\epsilon$ é chamada de seção identidade.

iv) Uma aplicação $i: G \rightarrow G$, tal que para toda flecha $g \in G, i(g) g=\epsilon_{s(g)}$ e $g i(g)=\epsilon_{t(g)}$. Esta aplicação $i$ é chamada de inversão. Para cada flecha $g \in G$, denotaremos $i(g)$ por $g^{-1}$.

De maneira equivalente, um grupoide é uma categoria pequena, na qual todos os morfismos são invertíveis. As propriedades acima envolvendo as aplicações estruturais, source $s$, target $t$, multiplicação $m$, seção identidade $\epsilon$ e inversão $i$, são consequências dos axiomas de uma categoria.

Um grupoide com conjunto de objetos $M$ e conjunto de flechas $G$ será denotado por $G \rightrightarrows M$ quando as aplicações $s$ e $t$ estiverem claras pelo contexto.

Definição 2.1.2. Um grupoide de Lie é um grupoide $G \rightrightarrows M$, onde $G$ e $M$ são variedades diferenciáveis, todas as aplicações estruturais são diferenciáveis e $s, t: G \rightarrow M$ são submersões sobrejetoras. 
O fato das aplicações estruturais $s$ e $t$ serem submersões sobrejetoras nos garante que o produto fibrado $G{ }_{s} \times{ }_{t} G$ é uma subvariedade de $G \times G$, então faz sentido que a multiplicação $m$ seja diferenciável. No caso de um grupoide de Lie, temos que a inversão $i$ é um difeomorfismo, pois é uma involução suave e, como $s \circ i=t$, basta que uma das aplicações $s$ ou $t$ seja submersão para que a outra também seja.

Exemplo 2.1.1. Um grupo de Lie $G$ é o mesmo que um grupoide sobre um conjunto com apenas um elemento $M=\{x\}$.

Exemplo 2.1.2. Seja $M$ uma variedade diferenciável. Definimos o grupoide unitário $M \rightrightarrows M$ com as seguintes aplicações estruturais:

i) $s, t=I d: M \rightarrow M$.

ii) $m: M_{s} \times_{t} M \rightarrow M$ dada por $m(x, x)=x$, onde $M_{s} \times_{t} M=\{(x, x) ; x \in M\}$.

iii) $\epsilon, i=I d: M \rightarrow M$.

Exemplo 2.1.3. Seja $M$ uma variedade diferenciável. Definimos o grupoide do par $M \times M \rightrightarrows M$ com as seguintes aplicações estruturais:

i) $s: M \times M \rightarrow M, s(x, y)=x$ e $t: M \times M \rightarrow M$ dada por $s(x, y)=y$.

ii) $m: M_{s} \times_{t} M \rightarrow M, m((z, w),(x, y))=(x, w)$, onde $M_{s} \times_{t} M=\{(z, w),(x, y) ; z=y\}$.

iii) $\epsilon: M \rightarrow M \times M, \epsilon(x)=(x, x)$.

iv) $i: M \times M \rightarrow M \times M, i(x, y)=(y, x)$.

Exemplo 2.1.4. Dada uma submersão sobrejetora $p: M \rightarrow N$, o produto fibrado $M_{p} \times{ }_{p} M$ tem uma estrutura de grupoide:

$$
M_{p} \times_{p} M \rightrightarrows M
$$

onde as aplicações $s$ e $t$ são, respectivamente, as projeções na primeira e segunda componentes. Em outras palavras, as aplicações estruturais são restriçôes a $M_{p}{ }_{p} M \subset M \times M$ das aplicaçôes do grupoide do par $M \times M \rightrightarrows M$. O grupoide $M_{p} \times{ }_{p} M \rightrightarrows M$ é chamado de grupoide de submersão.

Exemplo 2.1.5. Sejam $G$ um grupo de Lie e $M$ uma variedade diferenciável. Considere uma ação suave $\rho: G \times M \rightarrow M$ de $G$ em $M$. Então $G \times M$ é um grupoide de Lie sobre $M$ com as seguintes aplicações estruturais:

i) $s: G \times M \rightarrow M, s(g, x)=x$ e $t: G \times M \rightarrow M$ dada por $t(g, x)=\rho(g, x):=g \cdot x$.

ii) $m:(G \times M){ }_{s} \times{ }_{t}(G \times M) \rightarrow G \times M, m((g, x),(h, y))=(g h, y)$, onde $(G \times M){ }_{s} \times{ }_{t}(G \times M)=$ $\{(g, x),(h, y) ; x=h \cdot y\}$.

iii) $\epsilon: M \rightarrow G \times M, \epsilon(x)=(e, x)$, onde e denota o elemento identidade do grupo $G$.

iv) $i: G \times M \rightarrow G \times M, i(g, x)=\left(g^{-1}, g \cdot x\right)$.

Este grupoide é chamado de grupoide de transformação e denotado por $G \ltimes M \rightrightarrows M$. 
Exemplo 2.1.6. Se $M$ é uma variedade diferenciável, considere o conjunto $\Pi(M):=\{[\alpha] ; \alpha$ : $[0,1] \rightarrow M\}$ onde cada $[\alpha]$ é a classe de homotopia da curva $\alpha$ em $M$ com extremos fixos. Então $\Pi(M)$ é um grupoide de Lie sobre $M$, com as seguintes aplicaçôes estruturais:

i) $s: \Pi(M) \rightarrow M, s([\alpha])=\alpha(0)$ e $t: \Pi(M) \rightarrow M$ dada por $t([\alpha])=\alpha(1)$.

ii) Como $\Pi(M)_{s} \times{ }_{t} \Pi(M)=\{([\alpha],[\beta]) \in \Pi(M) \times \Pi(M) ; \alpha(0)=\beta(1)\}$, a multiplicação é dada por $m([\alpha],[\beta]):=[\alpha \cdot \beta]$, onde $\alpha \cdot \beta$ é a curva obtida através da concatenação de caminhos.

iii) $\epsilon: M \rightarrow \Pi(M), \epsilon(x)=[x]$, onde $[x]$ é a classe da curva constante igual a $x$.

iv) $i: \Pi(M) \rightarrow \Pi(M), i([\alpha])=\left(\left[\alpha^{-1}\right]\right)$, onde $\alpha^{-1}(r):=\alpha(1-r), r \in[0,1]$.

Este grupoide é chamado de grupoide fundamental de M. Como $s \times t: \Pi(M) \rightarrow M \times M$ é um recobrimento diferenciável, temos que $\Pi(M)$ possui uma estrutura de variedade diferenciável. Logo, $\Pi(M) \rightrightarrows M$ é um grupoide de Lie.

Exemplo 2.1.7. Considere um grupoide de Lie $G \rightrightarrows M$ com aplicações estruturais $s, t, m, i, \epsilon$. Definimos o grupoide de Lie TG $\rightrightarrows T M$ cujas aplicações estruturais são obtidas derivando as aplicações $s, t, m, i, \epsilon$, que serão denotadas por $T s, T t, T m, T i, T \epsilon$. Este grupoide é chamado de grupoide tangente de $G$.

Exemplo 2.1.8. Seja $E$ um fibrado vetorial sobre $M$. Considere $\mathrm{GL}(\mathrm{E})=\left\{\phi: E_{x} \rightarrow E_{y} ; \phi\right.$ é um isomorfismo e $x, y \in M\}$. Definimos o grupoide $\mathrm{GL}(\mathrm{E}) \rightrightarrows M$ com as seguintes aplicaçôes estruturais:

i) $s: \mathrm{GL}(E) \rightarrow M, s\left(\phi: E_{x} \rightarrow E_{y}\right)=x$ e $t: \mathrm{GL}(E) \rightarrow M, t\left(\phi: E_{x} \rightarrow E_{y}\right)=y$.

ii) $m: \mathrm{GL}(E){ }_{s} \times_{t} \mathrm{GL}(E) \rightarrow \mathrm{GL}(E), m\left(\left(\phi_{1}: E_{x} \rightarrow E_{y}\right),\left(\phi_{2}: E_{w} \rightarrow E_{z}\right)\right)=\left(\phi_{2} \circ \phi_{1}: E_{x} \rightarrow E_{z}\right)$, onde $\operatorname{GL}(E){ }_{s} \times{ }_{t} \operatorname{GL}(E)=\left\{\left(\left(\phi_{1}:\left(E_{x} \rightarrow E_{y}\right),\left(\phi_{2}: E_{w} \rightarrow E_{z}\right)\right) ; y=w\right\}\right.$.

iii) $\epsilon: M \rightarrow \mathrm{GL}(E), \epsilon(x)=\left(I d_{x}: E_{x} \rightarrow E_{x}\right)$.

iv) $i: \mathrm{GL}(E) \rightarrow \mathrm{GL}(E), i\left(\phi: E_{x} \rightarrow E_{y}\right)=\left(\phi^{-1}: E_{y} \rightarrow E_{x}\right)$.

Este grupoide é chamado de grupoide linear de E.

\subsection{Grupoides de Lie étale}

Definição 2.2.1. Um grupoide de Lie $G \rightrightarrows M$ é étale $\operatorname{se} \operatorname{dim} G=\operatorname{dim} M$.

Se $G \rightrightarrows M$ é um grupoide de Lie étale então as aplicações estruturais source e target são difeomorfismos locais.

Exemplo 2.2.1. O grupoide de Lie apresentado no exemplo (2.1.1) é étale se, e somente se, $G$ é discreto.

Exemplo 2.2.2. Para toda variedade diferenciável $M$, o grupoide unitário é étale.

Exemplo 2.2.3. O grupoide do par $M \times M \rightrightarrows M$ é étale se, e somente se, dim $M=0$.

Exemplo 2.2.4. O grupoide de transformação $G \ltimes M \rightrightarrows M$ é étale se, e somente se, $G$ é um grupo discreto. 
Definição 2.2.2. Sejam $G \rightrightarrows M$ um grupoide e $x \in M$. Definimos:

1. A órbita de $\mathbf{x}$ como o conjunto $t\left(s^{-1}\{x\}\right)=\{t(g) \in M ; g \in G$ e $s(g)=x\}$.

2. O espaço de órbitas do grupoide é o quociente de $M$ pela relação de equivalência $x \sim y$ se, e somente se, $x$ e y estão na mesma órbita. Ou seja, $M / G:=\left\{t\left(s^{-1}\{x\}\right) ; x \in M\right\}$.

3. O grupo de isotropia de $x$ é $G_{x}=\{g \in G ; s(g)=t(g)=x\}$.

Observação 2.2.1. Sejam $G \rightrightarrows M$ um grupoide e $x \in M$.

1. O grupo de isotropia de $x$ é um grupo de Lie.

2. A órbita de $x$ é uma subvariedade imersa de $M$.

3. Se $G$ é étale, então $s^{-1}\{x\}$ e $t^{-1}\{x\}$ são conjuntos discretos. Consequentemente, $G_{x}$ é discreto.

Definição 2.2.3. Um grupoide de Lie $G \rightrightarrows M$ é transitivo se a aplicação

$$
\begin{aligned}
& G \longrightarrow M \times M \\
& g \longmapsto(s(g), t(g))
\end{aligned}
$$

é uma submersão sobrejetora.

Exemplo 2.2.5. O grupoide do par $M \times M \rightrightarrows M$ é um grupoide de Lie transitivo.

Seja $G \rightrightarrows M$ um grupoide de Lie transitivo. Dado $(x, y) \in M \times M$, existe $g \in G$ tal que $s(g)=x$ e $t(g)=y$ pois $(s, t)$ é sobrejetora. Logo, existe apenas uma órbita, a saber o conjunto de objetos do grupoide.

\subsection{Representações de grupoides de Lie}

Nesta seção apresentaremos a noção de ação de um grupoide de Lie e exemplos. Introduziremos o conceito de morfismo entre grupoides de Lie e representação de grupoides de Lie.

Definição 2.3.1. Seja $G$ um grupoide de Lie sobre $M$ e seja $P$ uma variedade com uma aplicação diferenciável $a_{l}: P \rightarrow M$ chamada de aplicação momento. Uma ação à esquerda de $G$ em $P$ ao longo de $a_{l}$ é uma aplicação diferenciável $\mu: G_{s} \times_{a_{l}} P \rightarrow P$, a qual denotaremos por $\mu(g, p)=g \cdot p$, satisfazendo as seguintes condiçôes:

i) $g \cdot(h p)=(g h) \cdot p$ para todo $(g, h, p) \in G_{s} \times_{t} G_{s} \times_{a_{l}} P$.

ii) $\epsilon_{a_{l}(p)} \cdot p=p$ para todo $p \in P$.

iii) $a_{l}(g \cdot p)=t(g)$ para todo $(g, p) \in G_{s} \times_{a_{l}} P$.

Uma ação à esquerda de um grupoide $G$ em $P$ longo de $a_{l}$, será denotada por um diagrama da seguinte forma:

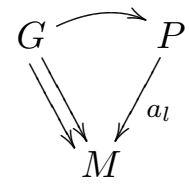


Naturalmente, a definição de ação à direita pode ser dada de forma análoga. Além disto, qualquer ação à esquerda pode ser transformada em uma ação à direita e, reciprocamente, uma ação à direita pode ser levada em uma ação à esquerda. Uma ação à direita de $H \rightrightarrows N$ em $P$ ao longo de $a_{r}: P \rightarrow N$ será denotada pelo diagrama a seguir:

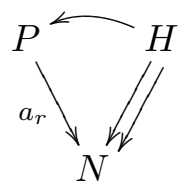

Exemplo 2.3.1. Se $G \rightrightarrows\{x\}$ é um grupo de Lie, a definição 2.3.1 se reduz ao conceito de ação de um grupo de Lie.

Sabemos que, se $G$ é um grupo de Lie e $g \in G$, então as translações à direita e à esquerda são difeomorfismos. No caso de grupoides temos uma definição análoga, porém estas definições não estarão definidas globalmente, como mostraremos a seguir. Considere um grupoide de Lie $G \rightrightarrows M$ e $g \in G$, definimos a translação à esquerda por $\mathbf{g}$ por

$$
\begin{aligned}
L_{g}: t^{-1}(s(g)) & \longrightarrow t^{-1}(t(g)) \\
h & \longmapsto g h .
\end{aligned}
$$

Similarmente, definimos a translação à direita por g por

$$
\begin{aligned}
R_{g}: s^{-1}(t(g)) & \longrightarrow s^{-1}(s(g)) \\
h & \longmapsto h g .
\end{aligned}
$$

Exemplo 2.3.2. A translação à esquerda é uma ação de $G$ em $G$ ao longo da aplicação $t$.

Exemplo 2.3.3. Seja $G$ um grupo de Lie agindo suavemente sobre as variedades $M$ e $N$. Então o grupoide de transformação $G \ltimes M \rightrightarrows M$ age ao longo da aplicação $a_{l}: N \rightarrow M$ da seguinte forma $n \cdot(g, x):=n \cdot g$, se e somente se, al é uma aplicação G-equivariante.

Definição 2.3.2. Seja $G$ um grupoide de Lie sobre $M$ agindo em uma variedade $P$ ao longo da aplicação $a_{l}: P \rightarrow M$. Dizemos que

i) a ação é livre $s e g \cdot p=p$ então $g=\epsilon\left(a_{l}(p)\right)$ para todo $(g, p) \in G_{t} \times{ }_{a_{l}} P$.

ii) a ação é própria se a função $G_{t} \times{ }_{a_{l}} G \rightarrow P_{a_{l}} \times{ }_{a_{l}} P$ é própria.

Observação 2.3.1. Se o grupoide de Lie G age em $P$ e a ação é livre e própria então o quociente de $P / G$ é uma variedade. Para maiores detalhes veja [8].

Definição 2.3.3. Sejam $G \rightrightarrows M$ e $H \rightrightarrows N$ dois grupoides de Lie. Um morfismo de grupoides de Lie entre $G$ e $H$ consiste em um par de aplicações diferenciáveis $\phi: G \rightarrow H$ e $\phi_{0}: M \rightarrow N$ que comutam com as aplicações estruturais de $G$ e de $H$. Ou seja, para cada $g \in G, x \in M$ e $\left(g_{1}, g_{2}\right) \in G_{s} \times{ }_{t} G$ temos

i) $\phi_{0}(s(g))=s(\phi(g))$ e $\phi_{0}(t(g))=t(\phi(g))$. 
ii) $\phi(\epsilon(x))=\epsilon\left(\phi_{0}(x)\right)$.

iii) $\phi\left(m\left(g_{1}, g_{2}\right)\right)=m\left(\phi\left(g_{1}\right), \phi\left(g_{2}\right)\right)$.

Neste caso, dizemos que $\phi: G \rightarrow H$ é um morfismo de grupoides de Lie cobrindo a aplicação $\phi_{0}: M \rightarrow N$.

Exemplo 2.3.4. Se $\phi$ é um morfismo entre grupoides de Lie da forma $G \rightrightarrows\{x\}$ e $H \rightrightarrows\{x\}$ então $\phi$ é um morfismo de grupos de Lie.

Exemplo 2.3.5. Seja $\phi: M \rightarrow N$ uma aplicação suave entre variedades. Temos que $\phi$ é um morfismo entre os grupoides $M \rightrightarrows M$ e $N \rightrightarrows N$ que cobre a própria aplicação $\phi$.

Exemplo 2.3.6. Se $G$ é um grupo de Lie agindo em uma variedade $M$ e $N$, e $f: M \rightarrow N$ é $G$-equivariante, ou seja, $f(g \cdot x)=g \cdot f(x)$, então obtemos um morfismo $\phi$ entre os grupoides de transformação $G \ltimes M \rightrightarrows M e G \ltimes N \rightrightarrows N$ dado por $\phi(g, x)=(g, f(x))$.

Definição 2.3.4. Uma bisseção de um grupoide de Lie $G \rightrightarrows M$ é uma aplicação suave $\tau: M \rightarrow G$ tal que $s \circ \tau=I d_{M}$ e $t \circ \tau: M \rightarrow M$ é um difeomorfismo.

Denotaremos o conjunto das bisseções de um grupoide de Lie $G \rightrightarrows M$ por $\operatorname{Bis}(G)$.

Observação 2.3.2. $\quad$ i) Se $\tau: M \rightarrow G$ é uma bisseção, então

$$
\begin{aligned}
& G \longrightarrow G \\
& g \longmapsto \tau(t(g)) g
\end{aligned}
$$

é um difeomorfismo.

ii) O conjunto $\operatorname{Bis}(G)$ é um grupo com a multiplicação definida da seguinte maneira,

$$
(\tau \cdot \delta)(x)=(\tau(t \circ \delta)(x)) \delta(x)
$$

para toda $\tau, \delta \in \operatorname{Bis}(G)$ e $x \in M$.

Exemplo 2.3.7. Se $G$ é um grupo de Lie de dimensão finita, então $\operatorname{Bis}(G)=G$.

Exemplo 2.3.8. Considere o grupoide do par $M \times M \rightrightarrows M$. Temos que, $\operatorname{Bis}(M \times M)=\operatorname{Diff}(M)$, onde $\operatorname{Diff}(M)$ denota o conjunto dos difeomorfismos em $M$.

Definição 2.3.5. Dado $G \rightrightarrows M$ um grupoide de Lie, uma bisseção local em $V \subset M$ é uma aplicação suave $\tau: V \rightarrow G$ tal que $s \circ \tau=I d_{V}$ e $t \circ \tau_{V}: V \rightarrow V$ é um difeomorfismo.

Note que, como $\tau$ é uma aplicação suave temos que $\tau^{-1}(U)$ é um aberto em $M$, onde $U$ é um aberto de $G$. Assim, obtemos uma definição equivalente para bisseção em termos de conjuntos abertos de $G$. Dizemos que uma bisseção local de um grupoide de Lie $G \rightrightarrows M$ é um subconjunto aberto $U$ de $G$ tal que $\left.s\right|_{U}$ e $\left.t\right|_{U}$ são injetoras. Para toda bisseção $U$ de um grupoide de Lie étale, temos um difeomorfismo $\tau_{U}: s(U) \rightarrow t(U)$ definido por $\tau_{U}=\left.t\right|_{U} \circ\left(\left.s\right|_{U}\right)^{-1}$. Além disto, bisseções de $G$ formam uma base para a topologia em $G$.

Para finalizar esta seção, introduziremos a seguir o conceito de representação de grupoides de Lie e daremos exemplos. Nossas principais referências são [5] e [2]. 
Definição 2.3.6. Sejam $G \rightrightarrows M$ um grupoide de Lie e $\pi_{E}: E \rightarrow M$ um fibrado vetorial de posto $k$. Uma representação de $G$ em $E$ é uma ação à esquerda $\rho: G{ }_{s} \times_{\pi_{E}} E \rightarrow E$, denotada por $\rho(g, e)=g \cdot e$, ao longo da projeção $\pi_{E}$, tal que todo $g \in G$ induz um isomorfismo linear $\rho_{g}: E_{s(g)} \rightarrow E_{t(g)}$ dado por $\rho_{g}(e)=g \cdot e$.

Exemplo 2.3.9. Representações do grupoide unitário associado a uma variedade diferenciável $M$ correspondem a fibrados vetoriais complexos sobre $M$.

Exemplo 2.3.10. Seja $G$ um grupo de Lie, temos que $G$ é um grupoide sobre um conjunto unitário $M=\{x\}$. Neste caso, recuperamos a noção de representação de grupo de Lie.

Exemplo 2.3.11. Se E é uma representação de $G \rightrightarrows M$ então $E_{x}$ é uma representação do grupo de isotropia $G_{x}$, para todo $x \in M$.

Exemplo 2.3.12. Dado um grupo de Lie $G$ agindo em uma variedade $M$, então podemos ver que as representações do grupoide de transformação

$$
G \ltimes M \rightrightarrows M
$$

são dadas por fibrados vetorias $G$-equivariantes sobre $M$. Lembramos que um fibrado $\pi_{E}: E \rightarrow M$ é G-equivariante quando a ação de $G$ em E é uma ação por automorfismos de E.

Exemplo 2.3.13. As representações do grupoide do par $M \times M \rightrightarrows M$ sobre um fibrado vetorial $E \rightarrow M$ correspondem a uma identificação de todas as fibras de E. Mais precisamente, fixado um ponto $x \in M$, para qualquer ponto $y \in M$ existe um único isomorfismo $\phi_{x y}: E_{x} \rightarrow E_{y}$. Com isto, temos bem definido um morfismo de fibrados $M \times E_{x} \rightarrow E$ dado por $(y, v) \mapsto \phi_{x y}(v)$. Como este é um isomorfismo, concluímos que E é um fibrado trivial. Portanto qualquer representação do grupoide do par é isomorfa a uma representação cuja a ação em cada fibra é constante.

Exemplo 2.3.14. Se $G \rightrightarrows M$ é um grupoide de Lie étale então $s, t$ são difeomorfismos locais. Considere os fibrados tangentes $\pi_{M}: T M \rightarrow M$ e $\pi_{G}: T G \rightarrow G$. Temos o seguinte diagrama,

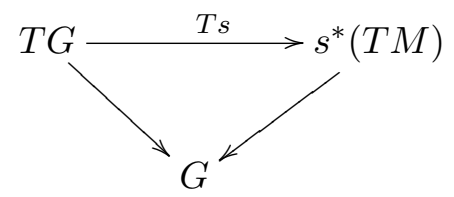

onde $s^{*}(T M)=G_{s} \times \pi_{M} T M$. A aplicação Ts é um isomorfismo de fibrados vetorias pois $G$ é étale. Seja $T G \rightrightarrows T M$ o grupoide tangente de $G$, apresentado em (2.1.7).

Definimos uma ação de $G$ em $T M$ ao longo da projeção $\pi_{M}: T M \rightarrow M$ dada por

$$
g \cdot v=T t\left((T s)^{-1}(g, v)\right)
$$

para todo $g \in G$ e todo $v \in T_{s(g)} M$. Como Tt e $(T s)^{-1}$ são morfismos de fibrados vetoriais, então a ação de $G$ em $T M$ ao longo de $\pi_{M}$ é uma representação.

Exemplo 2.3.15. Sejam $G \rightrightarrows M$ um grupoide de Lie e $\mathcal{O}$ uma órbita de $M$. Definimos $G_{\mathcal{O}}:=\{g \in G ; s(g), t(g) \in \mathcal{O}\}$ uma subvariedade de $G, G_{\mathcal{O}} \rightrightarrows \mathcal{O}$ é um grupoide de Lie e sua estrutura é induzida pela estrutura do grupoide de Lie $G \rightrightarrows M$. Considere os fibrados normais 


$$
\pi: N G_{\mathcal{O}}:=\frac{T G}{T G_{\mathcal{O}}} \longrightarrow G_{\mathcal{O}} \text { e } \pi_{\mathcal{O}}: N \mathcal{O}:=\frac{T M}{T \mathcal{O}} \longrightarrow \mathcal{O}
$$

A estrutura de grupoide de Lie do grupoide tangente TG $\rightrightarrows T M$ induz uma estrutura de grupoide de Lie em $N G_{\mathcal{O}} \rightrightarrows N \mathcal{O}$. Além disto, a aplicação $\pi$ é um morfismo de grupoides de Lie cobrindo $\pi_{\mathcal{O}}$. Definimos

$$
\begin{aligned}
\pi^{!}: N G_{\mathcal{O}} & \longrightarrow G_{\mathcal{O}}{ }_{s} \times_{\pi_{\mathcal{O}}} N \mathcal{O} \\
{[v] } & \longmapsto(\pi[v], T s([v]),
\end{aligned}
$$

para todo $[v] \in N G_{\mathcal{O}}$. Temos que $\pi^{!}$é um difeomorfismo. Portanto, os grupoides $N G_{\mathcal{O}} \rightrightarrows N \mathcal{O} e$ $G_{\mathcal{O}} \ltimes N \mathcal{O} \rightrightarrows N \mathcal{O}$ são isomorfos. Considere $\delta$ a inversa da aplicação $\pi^{!}$, definimos uma ação de $G_{\mathcal{O}}$ em $N \mathcal{O}$ ao longo de $\pi_{\mathcal{O}}$ por

$$
g \cdot([w]):=T t(\delta(g,[w]))
$$

para todo $[w] \in N \mathcal{O}$. Esta ação é uma representação de $G \mathcal{O}$ em $N \mathcal{O}$ e chamada de representação normal.

Exemplo 2.3.16. Considere o grupoide fundamental $\Pi(M) \rightrightarrows M$, apresentado em (2.1.6). Uma representação de $\Pi(M) \rightrightarrows M$ é equivalente a um fibrado vetorial $E \rightarrow M$ munido de uma conexão $\nabla$ de curvatura zero. De fato, para cada caminho ligando os pontos $x$ e y de $M$, a representação nos dá uma aplicação linear de $E_{x}$ em $E_{y}$, que será o transporte paralelo. O fato do transporte paralelo ser invariante por homotopia de caminhos diz que a conexão determinada por este transporte paralelo tem curvatura zero.

Observação 2.3.3. Uma representação de $G$ em E é o mesmo que um morfismo entre os grupoides de Lie $G$ e $\mathrm{GL}(\mathrm{E})$, como mostra o exemplo a seguir.

Exemplo 2.3.17. Sejam $E_{1}$ e $E_{2}$ duas representaçôes de $G \rightrightarrows M$. Denotaremos a ação de $G$ ao longo da projeção $\pi_{E_{1}}: E_{1} \rightarrow M$ por $\rho^{1}$ e a ação de $G$ ao longo da projeção $\pi_{E_{2}}: E_{1} \rightarrow M$ por $\rho^{2}$. Os fibrados vetoriais $E_{1} \oplus E_{2}$ e $E_{1} \otimes E_{2}$ ganham estrutura de $G$-representações da seguinte maneira, para todo $g \in G$ e todo $e_{1} \in E_{1}$ e $e_{2} \in E_{2}$ tais que $s(g)=\pi_{E_{1}}\left(e_{1}\right)=\pi_{E_{2}}\left(e_{2}\right)$, definimos as seguintes açôes:

$$
\begin{aligned}
E_{1 s(g)} \oplus E_{2 s(g)} & \longrightarrow E_{1 t(g)} \oplus E_{2 t(g)} \\
\left(e_{1}, e_{2}\right) & \longmapsto\left(\rho_{g}^{1}\left(e_{1}\right), \rho_{g}^{2}\left(e_{2}\right)\right),
\end{aligned}
$$

$e$

$$
\begin{aligned}
E_{1 s(g)} \otimes E_{2 s(g)} & \longrightarrow E_{1 t(g)} \otimes E_{2 t(g)} \\
\left(e_{1}, e_{2}\right) & \longmapsto \rho_{g}^{1}\left(e_{1}\right) \otimes \rho_{g}^{2}\left(e_{2}\right) .
\end{aligned}
$$

Portanto, os fibrados vetoriais $E_{1} \oplus E_{2}$ e $E_{1} \otimes E_{2}$ são representações de $G$.

Um morfismo entre representações $E$ e $F$ de um grupoide $G \rightrightarrows M$ é um morfismo $\phi: E \rightarrow F$ de fibrados vetoriais que cobre a identidade e satisfaz $\phi(g \cdot v)=g \cdot \phi(v)$, para todo $g \in G$ e $v \in E$ 
$\operatorname{com} s(g)=\pi(v)$.

Exemplo 2.3.18. Considere $G \rightrightarrows M$ um grupoide de Lie transitivo. Seja $E$ uma representação de $G$. Fixado $x \in M$, para cada $y \in M$, como $G$ é transitivo existe um único $g \in G$ tal que $s(g)=x$ e $t(g)=y$. Então, para cada $g \in G$ temos um isomorfismo $\phi$ entre as fibras $E_{x}$ e $E_{y}$. Definimos,

$$
\begin{aligned}
M \times E_{x} & \longrightarrow E \\
(y, v) & \longmapsto \phi(v),
\end{aligned}
$$

esta aplicação é um isomorfismo de fibrados vetoriais que cobre a identidade. Portanto E é um fibrado trivial.

Considere o grupoide de Lie $G_{x} \rightrightarrows\{x\}$. Seja $E_{x}$ uma representação de $G_{x}$. Para cada $g \in G$, iremos construir uma representação de $G$ no fibrado $M \times V$, onde $V$ é um espaço vetorial.

Se $g \in G_{x}$, temos

$$
\begin{aligned}
\{x\} \times E_{x} & \longrightarrow\{x\} \times E_{x} \\
(x, v) & \longmapsto(x, v)
\end{aligned}
$$

Como cada $\{x\} \times E_{x} \simeq E_{x}$, então $G_{x}$ age em $\{x\} \times E_{x}$.

Seja $h \in G$ tal que $s(h)=y$ e $t(h)=x$, temos $h g \in G_{x}$, logo existem aplicações $\psi_{h}: E_{x} \rightarrow E_{y}$ e $\psi_{h g}: E_{x} \rightarrow E_{x}$ e $\psi_{h g}=\phi_{h} \circ \phi_{g}$, portanto definimos

$$
\begin{aligned}
\{x\} \times E_{x} & \longrightarrow\{x\} \times E_{y} \\
(x, v) & \longmapsto(x, h g \cdot v),
\end{aligned}
$$

e obtemos uma ação de $G$ no fibrado vetorial $M \times E_{x}$. Com isto, provamos que $E$ é isomorfa a $E_{x}$, quando $G \rightrightarrows M$ é um grupoide transitivo.

\subsection{Equivalência de Morita}

Nesta seção apresentaremos a noção de equivalência de Morita entre grupoides de Lie. Mostraremos que grupoides de Lie Morita equivalentes têm "a mesma" teoria de representações.

Definição 2.4.1. Seja $G$ um grupoide de Lie sobre $M$. Uma variedade $P$ com uma ação de $G$ à direita ao longo de $a_{r}: P \rightarrow M$ é chamada de $G$-fibrado principal à direita sobre $B$ se

i) Existe uma submersão sobrejetora $\pi: P \rightarrow B$ tal que $\pi$ é $G$-invariante, i.e., $\pi(p \cdot g)=\pi(p)$, para todo $(p, g) \in P_{a_{r}} \times_{t} G$.

ii) A aplicação $P_{a_{r}} \times_{t} G \rightarrow P_{\pi} \times_{\pi} P$ dada por $(p, g)=(p, p \cdot g)$ é um difeomorfismo.

Se $P$ é um $G$-fibrado principal sobre $B$ com projeção $\pi: P \rightarrow B$, denotaremos tal estrutura através do seguinte diagrama: 


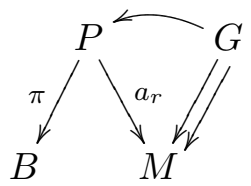

Observação 2.4.1. Pelo item ii), temos que as fibras da submersão $\pi$ são difeomorfas as órbitas da ação de $G$ sobre P. Além disto, a ação de $G$ em cada fibra é livre e transitiva.

Exemplo 2.4.1. Seja $G \rightrightarrows\{x\}$ um grupoide de Lie. Suponha que $\pi: P \rightarrow B$ é um $G$-fibrado principal à direita, então existe uma ação à direita de $G$ em $P$ ao longo de $a_{r}: P \rightarrow\{x\}$ dada por $\rho: P a_{a_{r}} \times{ }_{t} G \rightarrow P$. Como $a_{r}$ e $t$ são constantes temos $P_{a_{r}} \times_{t} G=P \times G$. Além disso, a aplicação $P \times G \rightarrow P_{\pi} \times_{\pi} P$ é um difeomorfismo. Portanto, recuperamos a noção de G-fibrado principal à direita para grupos de Lie.

Exemplo 2.4.2. Se $G \rightrightarrows M$ é um grupoide de Lie, consideramos a ação de $G$ em $G$ por multiplicação à direita, ao longo da aplicação $s: G \rightarrow M$. Então $G$ é um $G$-fibrado principal à direita sobre $M$.

i) O mapa $t: G \rightarrow M$ é uma submersão sobrejetora $G$-invariante;

ii) A aplicação $G_{s} \times_{t} G \rightarrow G_{t} \times_{t} G$ dada por $(h, g)=(h, h g)$ é um difeomorfismo, cuja função inversa é dada por $(g, k) \rightarrow\left(g, g^{-1} k\right)$, para todo $(g, k) \in G_{t} \times_{t} G$.

Na proposição a seguir definiremos o pullback de um $G$-fibrado principal. Além disto, mostraremos que este pullback é um $G$-fibrado principal.

Proposição 2.4.1. Sejam $G \rightrightarrows M$ um grupoide de Lie e $\pi: P \rightarrow B$ um G-fibrado principal à direita. Se $f: N \rightarrow B$ é uma aplicação suave então $f^{*}(P):=N_{f}{ }_{\pi} P$ é um $G$-fibrado principal à direita sobre $N$.

Demonstração. Como $G$ age à direita em $P$ ao longo da aplicação $a_{r}: P \rightarrow M$, definimos $\mu$ uma ação à direita de $G$ em $f^{*}(P)$ ao longo da aplicação $a_{r} \circ p r_{2}: f^{*}(P) \rightarrow M$ da seguinte maneira $\mu((n, p), g)=(n, p \cdot g)$ para todo $((n, p), g) \in\left(N_{f} \times{ }_{\pi} P\right)_{a_{r} \circ p r_{2}} \times_{p r_{1}} G$, onde $p r_{2}$ é a projeção na segunda componente.

i) Como $\pi$ é uma submersão, temos que $N_{f} \times{ }_{\pi} P$ é uma variedade. Além disto, o fato de $\pi$ ser uma submersão sobrejetora implica que a aplicação $p r_{1}: N_{f} \times_{\pi} P \rightarrow N$ é uma submersão sobrejetora. Para todo $(n, p) \in N_{f} \times_{\pi} P$, temos $p r_{1}((n, p), g)=p r_{1}(n, p \cdot g)=n=p r_{1}(n, p)$, logo $p r_{1}$ é $G$-invariante.

ii) A aplicação

$$
\begin{gathered}
\left(N_{f} \times_{\pi} P\right)_{a r \circ p r_{2}} \times_{p r_{1}} G \longrightarrow\left(N_{f} \times_{\pi} P\right)_{p r_{1}} \times_{p r_{1}}\left(N_{f} \times_{\pi} P\right) \\
((n, p), g) \longmapsto((n, p),(n, p \cdot g))
\end{gathered}
$$

é um difeomorfismo. Sua aplicação inversa é dada por $((n, p),(n, q)) \longmapsto((n, p), g)$, onde $g \in G$ é o único elemento tal que $p \cdot g=q$, o qual existe pelo item (ii). 
Definição 2.4.2. Sejam $G \rightrightarrows M$ e $H \rightrightarrows N$ dois grupoides de Lie. Um $(G$-H)-bifibrado consiste em uma variedade $P$, duas aplicações $a_{l}: P \rightarrow M$ e $a_{r}: P \rightarrow N$ satisfazendo:

i) O grupoide $G$ age à esquerda em $P$ ao longo de $a_{l}$.

ii) O grupoide $H$ age à direita em $P$ ao longo de $a_{r}$.

iii) A aplicação $a_{l}: P \rightarrow M$ é um $H$-fibrado principal com relação à ação de $H$.

iv) A aplicação $a_{r}$ é $G$-invariante, ou seja, $a_{r}(g \cdot p)=a_{r}(p)$ para todo $(g, p) \in G_{s} \times{ }_{a_{l}} P$.

v) As ações de $G$ e $H$ comutam, ou seja, $(g \cdot p) \cdot h=g \cdot(p \cdot h)$ para todo $(g, p, h) \in G_{s_{s} \times{ }_{l}} P_{a_{r}} \times_{t} H$

Se $P$ tiver uma estrutura de $(G-H)$-bifibrado, tal estrutura será denotada por

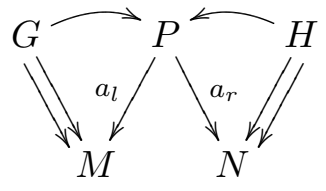

Ou simplesmente por $P: G \rightarrow H$.

Exemplo 2.4.3. Seja $G \rightrightarrows M$ um grupoide de Lie, o espaço total $G$, os mapas estruturais s e $t$ formam um $(G-G)$-bifibrado. De fato,

i) O grupoide $G$ age por multiplicação à esquerda em $G$ ao longo de $t: G \rightarrow M$.

ii) O grupoide $G$ age por multiplicação à direita em $G$ ao longo de $s: G \rightarrow M$.

iii) A aplicação $t: G \rightarrow M$ é um $G$-fibrado principal com relação à ação de $G$.

iv) A aplicação s é $G$-invariante, ou seja, $s(g \cdot p)=s(p)$ para todo $(g, p) \in G_{s} \times{ }_{t} G$.

v) As ações de $G$ e $G$ comutam, ou seja, $(g \cdot p) h=g(p \cdot h)$ para todo $(g, p, h) \in G_{s} \times{ }_{t} G_{s} \times{ }_{t} G$.

Proposição 2.4.2. Se $\phi: G \rightarrow H$ é um morfismo de grupoides de Lie cobrindo a aplicação $\phi_{0}: M \rightarrow N$ então $\phi_{0}^{*}(H):=M_{\phi_{0}}{ }{ }_{t} H$ é um $(G-H)$-bifibrado, que será denotado por $[\phi]$.

Demonstração. Considere $\phi: G \rightarrow H$ um morfismo de grupoides de Lie. Seja $t: H \rightarrow N$ o $H$-fibrado principal à direita apresentado no exemplo 2.4.3. Como $\phi_{0}: M \rightarrow N$ é suave, pela Proposição 2.4.1, o pullback de $t$ pela aplicação $\phi_{0}$, ou seja, $\phi_{0}^{*}(H)=M_{\phi_{0}} \times_{t} H$ é $H$-fibrado principal à direita.

i) $\mathrm{O}$ grupoide $H$ age à direita em $\phi_{0}^{*}(H)$ ao longo de $s \circ p r_{2}$.

ii) $\mathrm{O}$ grupoide $G$ age à esquerda em $M_{\phi_{0}} \times_{t} H$ ao longo de $p r_{1}: M_{\phi_{0}} \times_{t} H \rightarrow M$ através da aplicação

$$
\begin{aligned}
G_{s} \times_{p r_{1}}\left(M_{\phi_{0}} \times_{t} H\right) & \longrightarrow M_{\phi_{0}} \times_{t} H \\
(g,(s(g), h)) & \longmapsto(t(g), \phi(g) h) .
\end{aligned}
$$

iii) A aplicação $p r_{1}: \phi_{0}^{*}(H) \rightarrow M$ é um $H$-fibrado principal com relação à ação de $H$. 
iv) A aplicação $s \circ p r_{2}$ é $G$-invariante. De fato, dado $(g,(x, h)) \in G_{s} \times_{s o p r_{2}}\left(M_{\phi_{0}} \times_{t} H\right)$

$$
s \circ p r_{2}(g \cdot(x, h))=s \circ p r_{2}(t(g), \phi(g) h)=s \circ p r_{2}(\phi(g) h)=s(h)=s \circ p r_{2}(x, h) .
$$

v) Dado $\left(g, z, g_{1}\right) \in G_{s} \times_{p r_{1}}\left(M_{\phi_{0}} \times_{t} H\right)_{s o p r_{2}} \times_{t} H$, com $z=(x, h)$, temos

$$
(g \cdot z) \cdot g_{1}=(t(g), \phi(g) h) \cdot g_{1}=\left(t(g), \phi(g) h g_{1}\right)=g \cdot\left(x, h g_{1}\right)=g \cdot\left(z \cdot\left(g_{1}\right)\right) .
$$

Portanto, as ações de $G$ e $H$ comutam.

Se $\phi: G \rightarrow H$ é um morfismo de grupoides de Lie cobrindo a aplicação $\phi_{0}: M \rightarrow N$. Diremos que $[\phi]$ é o bifibrado induzido pelo morfismo $\phi$, ou simplesmente bifibrado induzido.

A proposição a seguir, nos mostra uma maneira natural de construirmos um novo bifibrado.

Proposição 2.4.3. Dados $P$ um $(G-H)$-bifibrado e $P^{\prime}$ um $(H$-K)-bifibrado, o quociente

$$
\left(P_{a_{r}} \times_{b_{l}} P^{\prime}\right) / H \text { é um (H-K)-bifibrado. }
$$

Demonstração. Sejam $P$ e $P^{\prime}$ dois bifibrados dados pelos diagramas a seguir,

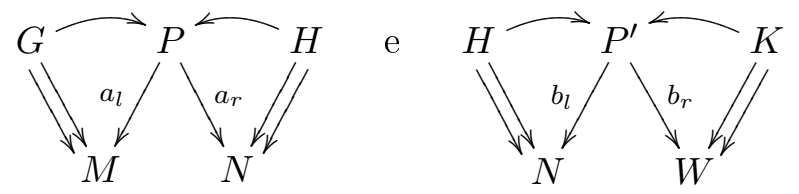

Construiremos um $(G-K)$-bifibrado denotado por $P \otimes P^{\prime}$, descrito pelo diagrama abaixo,

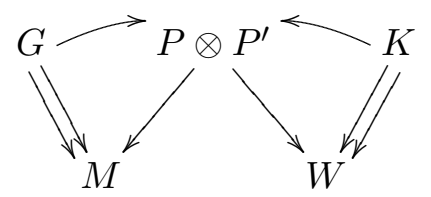

Primeiramente tomamos o produto fibrado $P_{a_{r}} \times{ }_{b_{l}} P^{\prime}$. Como $b_{l}$ é submersão, segue que tal produto fibrado é uma variedade. Além disto, $H$ age à direita da seguinte forma

$$
\begin{aligned}
\left(P_{a_{r}} \times_{b_{l}} P^{\prime}\right)_{a_{r} \circ p r_{1}} \times_{t} H & \longrightarrow P_{a_{r}} \times_{b_{l}} P^{\prime} \\
((p, q), h) & \longmapsto\left(p \cdot h, h^{-1} \cdot q\right) .
\end{aligned}
$$

Por outro lado, $G$ age por multiplicação à esquerda na primeira componente e $K$ age por multiplicação à direita na segunda componente. Como $P$ é um $(G-H)$-bifibrado e $P^{\prime}$ é um $(H-K)$-bifibrado pela definição de bifibrado temos que as ações de $G$ e $K$ comutam com a ação de $H$. Assim sendo, temos uma ação bem definida no quociente $\left(P_{a_{r}} \times{ }_{b_{l}} P^{\prime}\right) / H$.

Sabemos que $b_{l}: P^{\prime} \rightarrow N$ é um $K$-fibrado principal, portanto a aplicação $a_{l} \circ p r_{1}$ induz um fibrado principal no quociente,

$$
\overline{a_{l} \circ p r_{1}}:\left(P_{a_{r}} \times_{b_{l}} P^{\prime}\right) / H \longrightarrow M \text {. }
$$


Com isto, obtemos que $P \otimes P^{\prime}:=\left(P_{a_{r}} \times_{b_{l}} P^{\prime}\right) / H$ é um $(G-K)$-bifibrado:

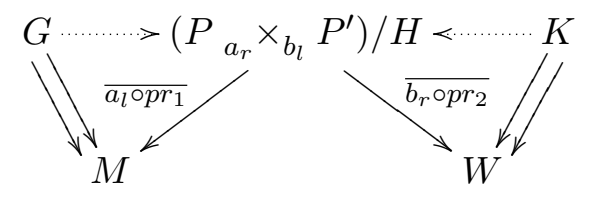

O $(G-K)$-bifibrado $\left(P_{a_{r}} \times_{b_{l}} P^{\prime}\right) / H$ apresentado pela proposição acima será denotado por $P \otimes P^{\prime}$ e quando for necessário explicitar que o quociente é dado pela ação do grupoide $H$. Usaremos a notação $P \otimes_{H} P^{\prime}$.

Definição 2.4.3. Dois $(G-H)$ bifibrados $P, P^{\prime}$ são ditos isomorfos se existe um difeomorfismo $\alpha: P \rightarrow P^{\prime},(G-H)$-equivariante, i.e., $\alpha(g \cdot p \cdot h)=g \cdot \alpha(p) \cdot h$ para todo $(g, p, h) \in G_{s} \times a_{l} P_{a_{r}} \times_{t} H$.

Exemplo 2.4.4. Se $\phi: M \rightarrow N$ é uma aplicação suave entre variedades então $\phi$ é um morfismo entre os grupoides $M \rightrightarrows M$ e $N \rightrightarrows N$. O bifibrado induzido por $\phi$ é o gráfico da aplicação $\phi$. Portanto, encontramos o seguinte bifibrado

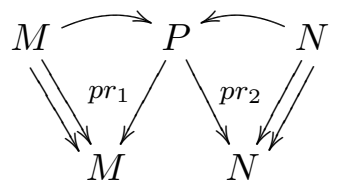

onde as ações de $M$ e de $N$ são triviais. Assim, se $\phi, \varphi: M \rightarrow N$ são duas aplicações suaves, existe um isomorfismo entre os bifibrados induzidos por $\phi, \varphi$ se, e somente se $\phi=\varphi$.

A próxima proposição afirma que a composição de bifibrados é associativa a menos de isomorfismo.

Proposição 2.4.4. Dados grupoides de Lie $G \rightrightarrows M, H \rightrightarrows N, K \rightrightarrows W$ e $L \rightrightarrows W$. Considere os bifibrados $P: G \rightarrow H, P^{\prime}: H \rightarrow K$ e $P^{\prime \prime}: K \rightarrow L$, então existe um isomorfismo de bifibrados $\alpha: P \otimes_{H}\left(P^{\prime} \otimes_{K} P^{\prime \prime}\right) \rightarrow\left(P \otimes_{H} P^{\prime}\right) \otimes_{K} P^{\prime \prime}$.

Demonstração. A aplicação

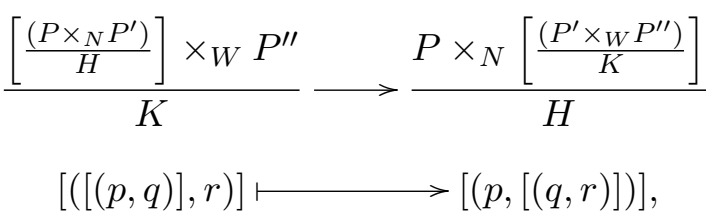

é um isomorfismo.

Proposição 2.4.5. Considere $G$ um grupoide de Lie, Id $: G \rightarrow G$ o isomorfismo identidade e $[I d]$ o bifibrado induzido. Para todo bifibrado $P: G \rightarrow H$ temos que $P \otimes[I d]$ é isomorfo a $P$.

Demonstração. Como $P \otimes[I d]=\left(G_{s} \times_{a_{l}} P\right) / G$. Definimos a aplicação

$$
\begin{aligned}
\alpha:\left(G_{s} \times_{a_{l}} P\right) / G & \longrightarrow P \\
(g, p) & \longmapsto g \cdot p
\end{aligned}
$$


Como $G$ age em $G_{s} \times a_{l} P$ ao longo de $s \circ p r_{1}=a_{l} \circ p r_{2}$ da seguinte maneira $(h, p) \cdot g=\left(h g, g^{-1} \cdot p\right)$, temos que a aplicação $\alpha$ é $(G-H)$-equivariante e sua inversa é dada por $p \longmapsto\left(\epsilon\left(a_{l}(p)\right), p\right)$. Segue que, $\alpha$ é isomorfismo entre $P$ e $P \otimes[I d]$.

Como a composição de bifibrados é associativa a menos de isomorfismo, o par (grupoides de Lie, bifibrados) não forma uma categoria, porém, o par (grupoides de Lie, classes de isomorfismos de bifibrados) forma uma categoria que será denotada por GRP.

Observação 2.4.2. Se $P: G \rightarrow H$ é $(G-H)$-bifibrado tal que a ação de $G$ também é principal, então podemos definir um $(H-G)$-bifibrado denotado $P^{-1}: H \rightarrow G$, transformando a ação de $G$ à esquerda em uma ação à direita e a ação de $H$ à direita em uma ação à esquerda. Então, pela Proposição 2.4.5, $P^{-1} \otimes P$ é isomorfo a $\left[I d_{H}\right]$ e $P \otimes P^{-1}$ é isomorfo a $\left[I d_{G}\right]$.

Definição 2.4.4. Dois grupoides de Lie $G$ e $H$ são Morita equivalentes se existe um $(G-H)$ bifibrado $P$ tal que a ação de $G$ à esquerda em $P$ é principal.

Exemplo 2.4.5. Sejam $G \rightrightarrows M$ um grupoide de Lie transitivo e fixado $x \in M$. Considere o grupoide de Lie $G_{x} \rightrightarrows\{x\}$, onde $G_{x}$ é o grupo de isotropia de $x$. Os grupoides de Lie $G \rightrightarrows M e$ $G_{x} \rightrightarrows\{x\}$ são Morita equivalentes. De fato, como $G$ é transitivo, a aplicação $(s, t): G \rightarrow M \times M$ é uma submersão sobrejetora. Considere $f: M \rightarrow M \times M$ dada por $f(y)=(x, y)$ e o seguinte diagrama

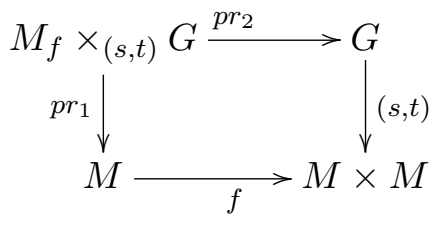

Temos que $s^{-1}\{x\} \simeq M_{f}{ }_{(s, t)} G$. Segue do fato que $(s, t)$ é uma submersão sobrejetora que $t: s^{-1}\{x\} \rightarrow M$ é uma submersão sobrejetora também. O grupoide de Lie $G$ age à esquerda por multiplicação em $s^{-1}\{x\}$ ao longo de $t$ e o grupoide de Lie $G_{x}$ age à direita por multiplicação em $s^{-1}\{x\}$ ao longo de s. Logo,

i) $t: s^{-1}\{x\} \rightarrow M$ é $G_{x}$-fibrado principal à direita sobre $M$.

ii) A aplicação

$$
\begin{aligned}
G_{s} \times_{t} s^{-1}\{x\} & \longrightarrow s^{-1}\{x\}_{s} \times_{s} s^{-1}\{x\} \\
(g, h) & \longmapsto(g h, h)
\end{aligned}
$$

é um difeomorfismo, cuja a inversa é dada por $(k, h) \longmapsto\left(k h^{-1}, h\right)$.

Portanto, $s^{-1}\{x\}$ é um $\left(G-G_{x}\right)$-bifibrado principal tal que a ação de $G$ à esquerda em $s^{-1}\{x\}$ é principal.

Exemplo 2.4.6. Considere $M$ uma variedade diferenciável. O grupoide do par $M \times M \rightrightarrows M$ é transitivo. Seja $x \in M$, temos $G_{x}=\{(x, x)\}$. Pelo exemplo anterior, os grupoides de Lie $M \times M \rightrightarrows$ $M e\{(x, x)\} \rightrightarrows\{x\}$ são Morita equivalentes. 
Exemplo 2.4.7. Sejam $N$ e $M$ variedades diferenciáveis. Considere $p: N \rightarrow M$ uma submersão sobrejetora e $N_{p} \times{ }_{p} N \rightrightarrows N$ o grupoide de submersão associado a $p$.

i) O grupoide de Lie $N_{p} \times{ }_{p} N$ age à esquerda em $N$ ao longo da aplicação identidade Id $: N \rightarrow N$ da seguinte maneira

$$
\begin{gathered}
N_{I d} \times_{p r_{2}} N \longrightarrow\left(N_{p} \times{ }_{p} N\right) \\
(x,(y, x)) \longmapsto y .
\end{gathered}
$$

ii) A submersão sobrejetora $p$ é $N_{p} \times{ }_{p} N$-invariante.

iii) A aplicação

$$
\begin{gathered}
M_{s} \times_{p} N \longrightarrow N_{I d} \times_{I d} N \\
(x, y) \longmapsto(y, y)
\end{gathered}
$$

é um difeomorfismo, cuja inversa é dada por $(z, z) \longmapsto(p(z), z)$.

Portanto, Id $: N \rightarrow N$ é $N$-fibrado principal à esquerda.

Segue que, os grupoides de Lie $\left(N_{p} \times{ }_{p} N \rightrightarrows N\right)$ e $(M \rightrightarrows M)$ são Morita equivalentes.

Exemplo 2.4.8. Considere o grupoide de transformação $G \ltimes M \rightrightarrows M$, cuja ação de $G$ em $M$ é livre e própria. Seja $\pi: M \rightarrow M / G$ a projeção canônica e o isomorfismo de grupoides de Lie

$$
\begin{gathered}
G \ltimes M \longrightarrow M_{\pi} \times_{\pi} M \\
(g, y) \longmapsto(y, g \cdot y) .
\end{gathered}
$$

Pelo exemplo 2.4.7, temos que os grupoides de Lie $\left(M_{\pi} \times_{\pi} M \rightrightarrows M\right)$ e $(M / G \rightrightarrows M / G)$ são Morita equivalentes. Segue que, $(G \ltimes M \rightrightarrows M)$ e $(M / G \rightrightarrows M / G)$ são Morita equivalentes.

\subsubsection{Invariância de Morita para representações}

O resultado principal desta subseção mostra que, se dois grupoides de Lie são Morita equivalentes então suas categorias de representações são equivalentes.

O conjunto das classes de isomorfismos de representações de um grupoide $G$ e os morfismos de representações formam uma categoria. Tal categoria será denotada por $\operatorname{Rep}(G)$.

Considere $G \rightrightarrows M$ e $H \rightrightarrows N$ dois grupoides de Lie e um $(G-H)$-bifibrado principal $P$, como mostra o diagrama abaixo

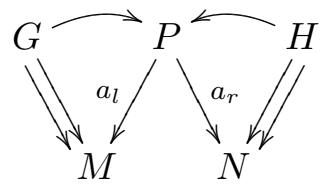

Seja $E$ uma representação de $H \rightrightarrows N$. Considere a ação de $H$ no fibrado vetorial pull-back $\left(a_{r}\right)^{*} E \rightarrow P$ dada por

$$
h \cdot(p, e)=\left(p \cdot h^{-1}, h \cdot e\right) .
$$

Temos uma estrutura de variedade diferenciável no quociente $\widetilde{E}=\left(a_{r}\right)^{*} E / H$ pois tal ação é livre e própria. Tomamos a aplicação $\widetilde{E} \rightarrow M$ dada por $[p, e] \mapsto a_{l}(p)$. Esta aplicação está bem definida 
pois $\left(p \cdot h^{-1}, h \cdot e\right) \in a_{r}^{*}(E)$. Afirmamos que esta aplicação define um fibrado vetorial sobre $M$. De fato, como a ação de $P$ é $H$ principal ao longo de $a_{l}$, se $a_{l}\left(p_{1}\right)=a_{l}\left(p_{2}\right)$ então existe um único $h \in H$ tal que $p_{1} \cdot h^{-1}=p_{2}$. Logo,

$$
\left[p_{1}, e_{1}\right]=\left[p_{1} h^{-1}, h \cdot e_{1}\right]=\left[p_{2}, h \cdot e_{2}\right]
$$

Com isto, podemos dar uma estrutura de espaço vetorial às fibras de $a_{l}$ fazendo:

$$
\begin{aligned}
{\left[p_{1}, e_{1}\right]+\left[p_{2}, e_{2}\right] } & :=\left[p_{2}, h \cdot e_{1}+e_{2}\right] \\
\lambda\left[p_{1}, e_{1}\right]: & :=\left[p_{1}, \lambda e_{1}\right],
\end{aligned}
$$

onde $a_{l}\left(p_{1}\right)=a_{l}\left(p_{2}\right)$ como descrito acima.

Vejamos agora que $\tilde{E}$ é localmente trivial. Todo ponto em $M$ é da forma $a_{l}(p)$, para algum $p \in P$. Considere uma base $e_{1}, \ldots, e_{n}$ de seções locais do fibrado $\left(a_{r}\right)^{*} E$. Definimos $\tilde{e}_{i}(y)=\left[\sigma(y), e_{i}(\sigma(y))\right]$ numa vizinhança de $a_{l}(p)$, onde $\sigma$ é uma seção de $\left(a_{r}\right)^{*} E$ tal que $\sigma\left(a_{l}(p)\right)=p$. Com isto obtemos seções locais $\tilde{e}_{i}$, com $i=1, \ldots, n$, que nos darão trivializações locais para $\widetilde{E}$. Note que, as mudanças de coordenadas serão feitas escrevendo os vetores de uma seção como combinação linear dos vetores de outra seção, logo a mudança de cartas é linear. Com isto, provamos que $\widetilde{E}=\left(a_{r}\right)^{*} E / H$ é um fibrado vetorial sobre $M$. Definimos uma ação do grupoide $G$ em $\widetilde{E}$ pela seguinte fórmula:

$$
g \cdot[p, e]=[g \cdot p, e] .
$$

A representação $\widetilde{E}$ será denotada por

$$
P \otimes_{H} E \longrightarrow M
$$

Dado $\phi: E \rightarrow F$ um morfismo de representações de $H$. Definimos um morfismo entre representações de $G$,

$$
\begin{aligned}
\operatorname{Rep}(\phi): P \otimes_{H} E & \longrightarrow P \otimes_{H} F \\
([p, e]) & \longmapsto[p, \phi(e)] .
\end{aligned}
$$

Ou seja, $\operatorname{Rep}(\phi)=I d_{P} \otimes \phi$. A aplicação $\operatorname{Rep}(\phi)$ está bem definida. De fato, se $[p \cdot h, e]=[p, h \cdot e]$, como $\phi$ é $H$-invariante temos

$$
\operatorname{Rep}(\phi)([p, h \cdot e])=[p, \phi(h \cdot e)]=[p, h \cdot \phi(e)]=[p \cdot h, \phi(e)]=\operatorname{Rep}(\phi)([p \cdot h, e]) .
$$

Portanto, dados dois grupoides de Lie $G$ e $H$ e um $(G$ - $H$ )-bifibrado $P$, temos um funtor entre as categorias $\operatorname{Rep}(H)$ e $\operatorname{Rep}(G)$ dado por $E \mapsto P \otimes_{H} E$. Se a ação de $G$ em $P$ é principal, este funtor é uma equivalência de categorias, como mostra o teorema a seguir.

Teorema 2.4.1. Se dois grupoides de Lie G e H são Morita equivalentes então o funtor

$$
\begin{aligned}
\operatorname{Rep}(H) & \longrightarrow \operatorname{Rep}(G) \\
E & \longrightarrow P \otimes_{H} E
\end{aligned}
$$


é uma equivalência de categorias.

Demonstração. Pela construção vista acima, sabemos que $P \otimes_{H} E \rightarrow M$ é uma representação do grupoide $G$. De maneira análoga, obtemos a seguinte representação de $H$,

$$
P \otimes_{G}\left(P \otimes_{H} E\right) \longrightarrow M
$$

e provaremos que esta representação é isomorfa a $E \rightarrow M$.

De fato, lembramos que o fibrado vetorial $P \otimes_{G}\left(P \otimes_{H} E\right)$ é o quociente

$$
\left(a_{l}\right)^{*}(\widetilde{E}) / G \longrightarrow N
$$

Definimos o morfismo de representações do grupoide $H$

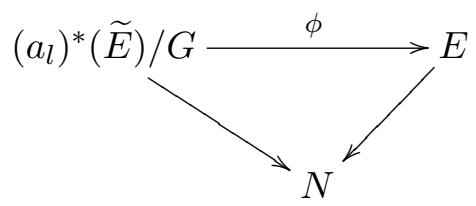

definido por $\phi([p,[q, e]])=\delta(p, q) \cdot e$, onde $\delta(p, q) \in H$ é o único elemento tal que $p \cdot \delta(p, q)=q$. A existência da aplicação $\delta$ segue do fato de que a ação de $H$ em $P$ é principal. Note que,

$$
\phi\left(\left[p,\left[q \cdot\left(h^{\prime}\right)^{-1}, h^{\prime} \cdot e\right]\right]\right)=\delta\left(x, y\left(h^{\prime}\right)^{-1}\right) \cdot\left(h^{\prime} \cdot e\right)=h\left(h^{\prime}\right)^{-1} \cdot\left(h^{\prime} \cdot e\right)=h \cdot e,
$$

obtemos que, $\phi$ está bem definida no quociente. Além disto, $\phi$ é um isomorfismo de fibrados vetoriais $H$-equivariante cobrindo a identidade em $N$. O que conclui esta demonstração.

Agora descreveremos alguns exemplos desta equivalência.

Exemplo 2.4.9. Considere os grupoides de Lie Morita equivalentes apresentados no exemplo 2.4.6, o grupoide do par $M \times M \rightrightarrows M$ é Morita equivalente ao grupoide $\{x\} \rightrightarrows\{x\}$. Pelo exemplo 2.3.13, uma representação $E$ do grupoide do par é isomorfa a uma representação trivial, $E \simeq M \times V$, onde $V$ é um espaço vetorial. Por outro lado, uma representação do grupoide $\{x\} \rightrightarrows\{x\}$ corresponde um espaço vetorial. Pelo Teorema 2.4.1 temos que, a categorias $\operatorname{Rep}(M \times M)$ e $\operatorname{Rep}(\{x\})$ são isomorfas, como apresentado no exemplo ??.

Exemplo 2.4.10. Seja $G \rightrightarrows M$ um grupoide de Lie transitivo Morita equivalente ao grupoide de Lie $G_{x} \rightrightarrows\{x\}$ apresentado no exemplo 2.4.5. Pelo Teorema 2.4.1, a categoria $\operatorname{Rep}(G \rightrightarrows M)$ é isomorfa à categoria $\operatorname{Rep}\left(G_{x} \rightrightarrows\{x\}\right)$.

Exemplo 2.4.11. Pelo exemplo 2.4.8, o grupoide de transformação $G \ltimes M \rightrightarrows M$ é Morita equivalente ao grupoide unitário sobre o espaço de órbitas, $M / G \rightrightarrows M / G$. Pelo exemplo 2.3.12, as representações do grupoide de transformação são dadas por fibrados G-equivariantes. Pelo Teorema 2.4.1 temos que, categoria $\operatorname{Rep}(G \ltimes M)$ é isomorfa à categoria $\operatorname{Rep}(M / G \rightrightarrows M / G)$.

Exemplo 2.4.12. O grupoide de submersão $N_{p} \times{ }_{p} N \rightrightarrows N$ é Morita equivalente ao grupoide unitário $M \rightrightarrows M$, conforme mostramos no exemplo 2.4.7. Pelo Teorema 2.4.1 temos que, a categoria $\operatorname{Rep}\left(N_{p} \times{ }_{p} N\right)$ é isomorfa à categoria $\operatorname{Rep}(M \rightrightarrows M)$. 


\section{Capítulo 3}

\section{Teorema de Serre-Swan para grupoides de Lie étale}

Neste capítulo introduziremos a definição de álgebra de convolução de um grupoide de Lie, considerando [11] e [13] como principais referências. Mostraremos que o espaço das seções de toda representação de um grupoide de Lie étale possui uma estrutura natural de módulo sobre a álgebra de convolução deste grupoide. Em seguida, estudaremos a categoria $\operatorname{Mod}(G)$, dos módulos de tipo finito e posto constante sobre tais álgebras e demonstraremos o Teorema de Serre-Swan para grupoides de Lie étale, que estabelece uma equivalência de categorias entre $\operatorname{Rep}(G)$ e $\operatorname{Mod}(G)$, estendendo o Teorema de Serre-Swan para variedades diferenciáveis. Para esta parte seguimos [5].

\section{1 Álgebra de convolução de um grupoide de Lie étale}

Considere $G$ um grupo de Lie e $\mu$ uma medida de Haar em $G$. Sejam $\varphi, \psi \in C_{c}^{\infty}(G)$, o produto de convolução de $\varphi$ por $\psi$ é definido da seguinte forma

$$
(\varphi * \psi)(g):=\int_{h \in G} \varphi\left(g h^{-1}\right) \psi(h) d \mu_{h}
$$

É natural desejarmos generalizar essa definição para grupoides de Lie. Explicaremos como isto será feito. Seja $G$ um grupoide de Lie sobre $M$. Considere o espaço vetorial $C_{c}^{\infty}(G)$ das funções diferenciáveis com suporte compacto. Dados $\varphi, \psi \in C_{c}^{\infty}(G)$, e $g \in G$, queremos definir o produto $(\varphi * \psi)(g)$. Analisamos todos os possíveis pares $\left(g_{1}, g_{2}\right) \in G_{s} \times_{t} G$ tais que $g=g_{1} g_{2}$ e integramos o produto $\varphi\left(g_{1}\right) \psi\left(g_{2}\right)$,

$$
(\varphi * \psi)(g)=\int_{\left\{\left(g_{1}, g_{2}\right) \in G_{s} \times{ }_{t} G ; g=g_{1} g_{2}\right\}} \varphi\left(g_{1}\right) \psi\left(g_{2}\right)(\ldots)
$$

onde (...) precisa ser uma medida em $\left\{\left(g_{1}, g_{2}\right) \in{ }_{s} \times_{t} ; g=g_{1} g_{2}\right\}$. Ao escrevermos $g_{1} g_{2}=g$ como $g_{2}=g_{1}^{-1} g$, alteramos o domínio de integração para $\left\{g_{1} \in G ; s\left(g_{1}^{-1}\right)=t(g)=t\left(g_{1} g_{2}\right)=t\left(g_{1}\right)\right\}$, e obtemos

$$
\varphi * \psi(g)=\int_{g_{1} \in t^{-1}(t(g))} \varphi\left(g_{1}\right) \psi\left(g_{2}\right)(\ldots)
$$

onde $(\ldots)$ precisa ser uma medida em $t^{-1}(t(g))$. Para que este produto esteja bem definido é neces- 
sário que o grupoide de Lie $G$ admita um sistema de Haar, que será nossa próxima definição.

Definição 3.1.1. Dado um grupoide $G \rightrightarrows M$, um sistema de Haar é uma familia de medidas $\left\{\lambda^{x}\right\}_{x \in M}$ satisfazendo as seguintes condiçôes:

i) O suporte de $\lambda^{x}$ é $t^{-1}(x)$.

ii) Para toda $\phi \in C_{c}^{\infty}(G)$, a aplicação

$$
\begin{aligned}
& M \longrightarrow \mathbb{R} \\
& x \longmapsto \int \phi d \lambda^{x}
\end{aligned}
$$

é contínua.

iii) Para todo $g \in G$ e toda $\phi \in C_{c}^{\infty}(G)$,

$$
\int_{h \in t^{-1}(s(g))} \phi(g h) d \lambda^{s(g)}=\int_{h \in t^{-1}(t(g))} \phi(h) d \lambda^{t(g)} .
$$

Ou seja, o sistema de Haar é invariante por translação à esquerda.

Exemplo 3.1.1. Considere o grupoide de Lie $G \rightrightarrows\{x\}$. Como a fibra $t^{-1}(\{x\})$ coincide com o grupo de Lie $G$, e como todo grupo de Lie admite uma medida de Haar, neste caso, temos que um sistema de Haar é formado por apenas uma medida.

Exemplo 3.1.2. Considere um grupoide de Lie étale $G \rightrightarrows M$, como as $t$-fibras são discretas, o sistema de Haar será dado pela medida da contagem $\mu$, ou seja, $\mu\left(t^{-1}(t(g))\right)$ é igual ao número de elementos de $t^{-1}(t(g))$, onde $g \in G$.

Exemplo 3.1.3. Considere o grupoide do par $M \times M \rightrightarrows M$, um sistema de Haar será dado pela medida de Borel. No caso, em que $M$ possui uma quantidade finita de elementos, temos que o sistema de Haar será dado pela medida da contagem.

Todo grupoide de Lie admite um sistema de Haar. A demonstração deste resultado pode ser encontrada em [11].

Dado um sistema de Haar $\left\{\lambda^{x}\right\}_{x \in M}$ em $G \rightrightarrows M$, definimos o produto de convolução em $C_{c}^{\infty}(G)$ por:

$$
(\varphi * \psi)(g)=\int_{g_{1} \in t^{-1}(t(g))} \varphi\left(g_{1}\right) \psi\left(g_{1}^{-1} g\right) d \lambda^{t(g)}
$$

onde $\varphi, \psi \in C_{c}^{\infty}(G)$ e $g \in G$.

O seguinte resultado é fundamental neste trabalho.

Teorema 3.1.1. Considere $G$ um grupoide de Lie. O espaço vetorial $C_{c}^{\infty}(G)$ munido do produto de convolução é uma álgebra associativa.

Demonstração. Mostraremos que o produto de convolução está bem definido. Dados $\varphi, \psi \in C_{c}^{\infty}(G)$ e $g \in G$, se existe $g_{1} \in G$ tal que $\varphi\left(g_{1}\right)$ e $\psi\left(g_{1}^{-1} g\right)$ são zero então $(\varphi * \psi)(g)$ é zero. Ou seja, se $g_{1} \notin \operatorname{supp}(\varphi)$ e $g_{1}^{-1} g \notin \operatorname{supp}(\psi)$ então $g \notin \operatorname{supp}(\varphi * \psi)$. Dessa forma, $\operatorname{supp}(\varphi * \psi)$ está contido no conjunto compacto $\operatorname{supp}(\varphi) \cap \operatorname{supp}(\psi)$. Portanto, $\varphi * \psi$ possui suporte compacto. Resta verificar que $\varphi * \psi$ é suave. Como $G_{s} \times{ }_{t} G$ é um conjunto fechado em $G \times G$, a aplicação suave 


$$
\begin{aligned}
\alpha: G_{s} \times{ }_{t} G & \longrightarrow \mathbb{C} \\
\left(g, g_{1}\right) & \longmapsto \psi\left(g_{1}^{-1} g\right)
\end{aligned}
$$

pode ser estendida para uma aplicação suave e limitada $\tilde{\alpha}$ em $G \times G$. Assim, obtemos as seguintes aplicações suaves

$$
\begin{aligned}
& \beta: G \longrightarrow C_{c}^{\infty}(G) \\
& \left(g_{1}\right) \longmapsto \varphi\left(g_{1}\right) \tilde{\alpha}\left(g, g_{1}\right) .
\end{aligned}
$$

$\mathrm{E}$

$$
\begin{aligned}
\gamma: G \times M & \longrightarrow \mathbb{C} \\
(g, x) & \longmapsto \int \varphi\left(g_{1}\right) \tilde{\alpha}\left(g, g_{1}\right) d \lambda^{x}\left(g_{1}\right)
\end{aligned}
$$

De forma que, $\varphi * \psi$ é a restrição da aplicação $\gamma$ quando $x=s(g)=t\left(g_{1}\right)$. Portanto, $\varphi * \psi \in C_{c}^{\infty}(G)$. Agora, mostraremos que o produto de convolução é associativo. Dados $\varphi, \psi, \phi \in C_{c}^{\infty}(G)$ e $g \in G$, por definição do produto de convolução temos,

$$
\begin{aligned}
& (\varphi *(\psi * \phi))(g)=\int \varphi\left(g_{1}\right)(\psi * \phi)\left(g_{1}^{-1} g\right) d \lambda^{t(g)}\left(g_{1}\right) \\
= & \int \varphi\left(g_{1}\right)\left(\int \psi\left(g_{2}\right) \phi\left(g_{2}^{-1}\left(g_{1}^{-1} g\right)\right) d \lambda^{t\left(g_{1}^{-1} g\right)}\left(g_{2}\right)\right) d \lambda^{t(g)}\left(g_{1}\right) \\
= & \left.\iint \varphi\left(g_{1}\right) \psi\left(g_{2}\right) \phi\left(g_{2}^{-1}\left(g_{1}^{-1} g\right)\right) d \lambda^{s\left(g_{1}\right)}\left(g_{2}\right)\right) d \lambda^{t(g)}\left(g_{1}\right) \\
= & \iint \varphi\left(g_{1}\right) \psi\left(g_{2}\right) \phi\left(g_{2}^{-1} g_{1}^{-1} g\right) d \lambda^{t\left(g_{1}^{-1}\right)}\left(g_{1}\right) d \lambda^{t(g)}\left(g_{2}\right)
\end{aligned}
$$

Como o sistema de Haar é invariante por translação, escrevemos a equação acima da seguinte forma,

$$
\iint \varphi\left(g_{1}\right) \psi\left(g_{1}^{-1} g_{2}\right) \phi\left(g_{2}^{-1} g\right) d \lambda^{t\left(g_{2}\right)}\left(g_{1}\right) d \lambda^{t(g)}\left(g_{2}\right)=\int \varphi * \psi\left(g_{2}\right) \phi\left(g_{2}^{-1} g\right) d \lambda^{t(g)}\left(g_{2}\right)=(\varphi * \psi) * \phi(g) .
$$

Definição 3.1.2. A álgebra de convolução de um grupoide de Lie $G \rightrightarrows M$ é definida como o espaço vetorial $C_{c}^{\infty}(G)$ munido do produto

$$
(\varphi * \psi)(g)=\int_{g_{1} \in t^{-1}(t(g))} \varphi\left(g_{1}\right) \psi\left(g_{1}^{-1} g\right) d \lambda^{t(g)}
$$

para todo $g \in G$, onde $\varphi$ e $\psi$ são funçôes em $C_{c}^{\infty}(G)$ e $\left\{\lambda^{t(g)}\right\}$ é um sistema de Haar.

Dados dois sistemas de Haar em $G \rightrightarrows M$, temos que as álgebras de convolução de $G$ referente a esses dois sistemas de Haar são isomorfas como álgebras. 
Em um grupoide de Lie étale as $t$-fibras são subvariedades discretas de $G$, assim o produto de convolução é dado por:

$$
(\varphi * \psi)(g)=\sum_{g_{1} \in t^{-1}(t(g))} \varphi\left(g_{1}\right) \psi\left(g_{1}^{-1} g\right)
$$

para todo $g \in G$ onde $\varphi$ e $\psi$ são funções em $C_{c}^{\infty}(G)$.

Exemplo 3.1.4. Se G é um grupo de Lie então recuperamos a definição de produto de convolução para grupos de Lie apresentada em (3.1).

Exemplo 3.1.5. Seja $M$ uma variedade diferenciável. Considere o grupoide unitário $M \rightrightarrows M$. Dados $\varphi, \psi \in C_{c}^{\infty}(M \rightrightarrows M)$ e $g \in M$. Como o mapa $t$ é a Id, pela definição de produto de convolução temos,

$$
(\varphi * \psi)(g)=\int_{g_{1} \in\{g\}} \varphi\left(g_{1}\right) \psi\left(g_{1}^{-1} g\right) d \mu^{g}=\varphi(g) \psi(g),
$$

onde $\left\{\mu^{g}\right\}_{g \in M}$ é um sistema de Haar, dado pela medida da contagem. Portanto, a álgebra de convolução $C_{c}^{\infty}(M \rightrightarrows M)$ é precisamente a álgebra de funções $C_{c}^{\infty}(M)$ e o produto de convolução é a multiplicação usual.

Exemplo 3.1.6. Seja $M$ um conjunto com n elementos. Considere o grupoide do par $M \times M \rightrightarrows M$. Dado $(x, y) \in M \times M$, definimos,

$$
\begin{aligned}
\Phi: C_{c}^{\infty}(M \times M) & \longrightarrow M_{n \times n}(\mathbb{C}) \\
\varphi & \longmapsto[\varphi(x, y)]:=\left[A_{x y}\right] .
\end{aligned}
$$

onde $1 \leq x, y \leq n$. A aplicação $\Phi$ é um isomorfismo de álgebras.

i) Sejam $\varphi, \psi \in C_{c}^{\infty}(M \times M) e(x, y),(z, w) \in M \times M$ tais que $\Phi(\varphi)=\Phi(\psi)$. Temos,

$$
\begin{aligned}
\Phi(\varphi)=\Phi(\psi) & \Longrightarrow[\varphi(x, y)]=[\psi(z, w)] \\
& \Longrightarrow\left[A_{x y}\right]=\left[A_{z w}\right] \\
& \Longrightarrow A_{x, y}=B_{z, w}
\end{aligned}
$$

para todo $x, y, z, w \in\{1, \ldots, n\}$. Portanto, $\Phi$ é injetora.

ii) Dado $\left[A_{x y}\right] \in M_{n \times n}(\mathbb{C})$. Seja $(x, y) \in M \times M$, e tomamos $\varphi(x, y)=A_{x, y}$. Logo, $\left[A_{x y}\right]=$ $[\varphi(x, y)]$. Portanto, existe $\varphi \in C_{c}^{\infty}(M \times M)$ tal que $\Phi(\varphi)=\left[A_{x y}\right]$. Então $\Phi$ é sobrejetora.

iii) Dados $\varphi, \psi \in C_{c}^{\infty}(G)$ e $(x, y) \in M \times M$. Como este grupoide é étale, pela definição de produto de convolução temos

$$
\begin{gathered}
(\varphi * \psi)(x, y)=\sum_{(z, y) \in t^{-1}(t(x, y))} \varphi(z, y) \psi((y, z)(x, y)) \\
=\sum_{(z, y) \in t^{-1}(y)} \varphi(z, y) \psi(x, z), \text { pois }(y, z)(x, y)=(x, z) e(t(x, y))=y .
\end{gathered}
$$


Assim, o produto de convolução é dado por

$$
\sum_{z=1}^{n} \varphi(z, y) \psi(x, z)=\left[A_{z y}\right]\left[B_{x z}\right]
$$

Portanto, $\Phi$ é um morfismo de álgebras.

Concluímos que, a álgebra de convolução $C_{c}^{\infty}(M \times M \rightrightarrows M)$ coincide com a álgebra de matrizes $n \times n$ com coeficientes complexos e o produto de convoluçâa coincide com o produto de matrizes.

Observação 3.1.1. A álgebra de convolução $C_{c}^{\infty}(G)$ de um grupoide de Lie não necessariamente tem unidade.

i) Seja $G \rightrightarrows M$ um grupoide de Lie tal que $G$ é uma variedade compacta, a aplicação identicamente igual 1 , tem suporte compacto. Portanto, a álgebra de convolução $C_{c}^{\infty}(G)$ tem unidade.

ii) Considere o grupoide de Lie $\mathbb{R} \rightrightarrows \mathbb{R}$. A álgebra de convolução $C_{c}^{\infty}(\mathbb{R} \rightrightarrows \mathbb{R})=C_{c}^{\infty}(\mathbb{R})$ não tem unidade.

No que resta do presente capítulo, assumiremos que $G \rightrightarrows M$ é um grupoide de Lie étale, a menos que o contrário seja dito.

Proposição 3.1.1. O espaço vetorial $C_{c}^{\infty}(M)$ é uma subálgebra de $C_{c}^{\infty}(G)$. Em particular, todo $C_{c}^{\infty}(G)$-módulo pode ser visto como $C_{c}^{\infty}(M)$-módulo.

Demonstração. Como $M$ é uma subvariedade mergulhada de $G$ e sendo $G$ étale, temos que $M$ é um aberto de $G$. Portanto, para toda função $f \in C_{c}^{\infty}(M)$ existe

$$
\tilde{f}: G \longrightarrow \mathbb{C} \quad \text { tal que }\left.\quad \tilde{f}\right|_{M} \equiv f \quad \text { e }\left.\quad \tilde{f}\right|_{G / M} \equiv 0
$$

Portanto, $\tilde{f} \in C_{c}^{\infty}(G)$. Assim, obtemos um morfismo de álgebras injetor

$$
\begin{gathered}
C_{c}^{\infty}(M) \longrightarrow C_{c}^{\infty}(G) \\
f \longmapsto \tilde{f} .
\end{gathered}
$$

\subsection{Módulos de tipo finito e módulos de posto constante}

Sejam $M$ uma variedade diferenciável e $C_{c}^{\infty}(M)$ a álgebra de funções suaves com suporte compacto sobre $M$. A categoria $\operatorname{Vect}(M)$ dos fibrados vetoriais suaves sobre $M$ coincide com a categoria das representações do grupoide unitário $M \rightrightarrows M$.

Teorema 3.2.1. Seja $\pi_{E}: E \rightarrow M$ uma representação de $G \rightrightarrows M$. O espaço vetorial $\Gamma_{c}^{\infty}(E)$ das seções suaves de E com suporte compacto possui uma estrutura natural de módulo sobre a álgebra de convolução $C_{c}^{\infty}(G)$.

Demonstração. Definimos a aplicação bilinear $C_{c}^{\infty}(G) \times \Gamma_{c}^{\infty}(E) \longrightarrow \Gamma_{c}^{\infty}(E)$ dada por,

$$
(\varphi \sigma)(y)=\sum_{g \in t^{-1}(y)} \varphi(g)(g \cdot \sigma(s(g)))
$$


onde $\varphi \in C_{c}^{\infty}(G)$ e $\sigma \in \Gamma_{c}^{\infty}(E)$. Como $\varphi \in C_{c}^{\infty}(G)$ possui suporte compacto e as fibras $t^{-1}(y)$ são discretas, temos que existe apenas uma quantidade finita de elementos $g \in t^{-1}(x)$ com $\varphi(g) \neq 0$ para cada $x \in M$. Portanto, $\varphi \sigma$ é uma seção do fibrado vetorial $E$ sobre $M$.

Mostraremos agora que, $\varphi \sigma$ é uma seção suave com suporte compacto do fibrado vetorial $E$ sobre $M$.

Considere $\left\{\phi_{i}\right\}_{\{i \in I\}}$ uma partição da unidade subordinada a cobertura $\left\{U_{i}\right\}$ de $G$, onde $U_{i}$ são bisseções de $G$. Temos que cada $\phi_{i}$ é positiva, tem suporte compacto contida na bisseção $U_{i}$ e $\sum_{i=1}^{n} \phi_{i}=1$. Definimos $\varphi_{i}=\phi_{i} \varphi$, então

$$
\varphi=\sum_{i=1}^{n} \varphi_{i}=\sum_{i=1}^{n} \phi_{i} \varphi
$$

onde cada $\varphi_{i} \in C_{c}^{\infty}(G)$ possui suporte contido na bisseção $U_{i}$, com $i \in I$. Assim, podemos considerar sem perda de generalidade que, $\varphi=\varphi_{i}$ e seu suporte está contido na bisseção $U=U_{i}$ para algum $i$.

Considere $V=s(U), W=t(U)$ e o seguintes diagramas comutativos

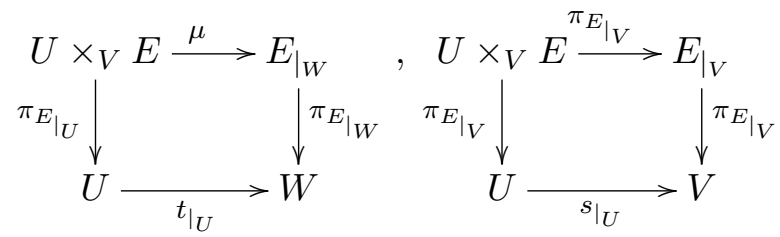

onde $\mu$ é a ação da representação $E$. Unindo os diagramas comutativos em (3.6), obtemos o seguinte diagrama comutativo,

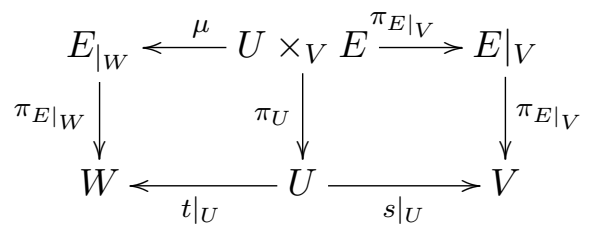

Note que, as aplicações $\mu$ e $\pi_{E_{\left.\right|_{V}}}$ são isomorfismos de fibrados vetoriais que cobrem os difeomorfismos $\left.t\right|_{U},\left.s\right|_{U}$, respectivamente. Definimos,

$$
\tau_{U}:=\mu \circ \pi_{E_{\left.\right|_{V}}}^{-1}: E_{\left.\right|_{V}} \longrightarrow E_{\left.\right|_{W}} .
$$

A função $\tau_{U}$ é um isomorfismo de fibrados vetoriais que cobre o difeomorfismo $\delta_{U}:=\left(\left.t\right|_{U}\right) \circ\left(\left.s\right|_{U}\right)^{-1}$ : $V \longrightarrow W$.

Seja $\sigma_{V} \in \Gamma_{c}^{\infty}\left(E_{\left.\right|_{V}}\right)$. Definimos

$$
\sigma_{U}^{\prime}:=\tau_{U} \circ \sigma_{V} \circ\left(\delta_{U}\right)^{-1}: W \longrightarrow E_{\left.\right|_{W}} .
$$

A função $\sigma_{U}^{\prime}$ é uma seção suave do fibrado vetorial $\left.E\right|_{W}$.

Como $\varphi$ tem suporte compacto contido em $U$, então $\varphi \circ\left(\left.t\right|_{U}\right)^{-1}$ possui suporte compacto contido em $W$, temos

$$
\begin{aligned}
\left(\left(\varphi \circ\left(\left.t\right|_{U}\right)^{-1}\right) \circ \sigma_{U}^{\prime}\right): W & \longrightarrow E_{\left.\right|_{W}} \\
(y) & \longmapsto\left(\varphi \circ\left(\left.t\right|_{U}\right)^{-1}\right)(y) \sigma_{U}^{\prime}(y) .
\end{aligned}
$$


Como $\left.t\right|_{U}$ é um difeomorfismo e $U$ é uma bisseção temos $(\varphi \sigma)(t(g))=\varphi(g)(g \cdot(\sigma(s(g))))$.

Segue que,

$$
(\varphi \sigma)(y)=\left(\varphi \circ\left(\left.t\right|_{U}\right)^{-1}\right)(y) \sigma_{U}^{\prime}(y) .
$$

Ou seja, para toda $\varphi \in C_{c}^{\infty}(G)$ e para toda $\sigma \in \Gamma_{c}^{\infty}(E),(\varphi \sigma) \in \Gamma_{c}^{\infty}(E)$ e tem suporte compacto contido em $W$.

Mostraremos que a aplicação definida em (3.5) é uma ação de $C_{c}^{\infty}(G)$ em $\Gamma_{c}^{\infty}(E)$.

i) $\operatorname{Dados} \varphi \in C_{c}^{\infty}(G), \sigma_{1}, \sigma_{2} \in \Gamma_{c}^{\infty}(E)$ e $y \in M$,

$$
\begin{aligned}
\left(\varphi\left(\sigma_{1}+\sigma_{2}\right)\right)(y) & =\sum_{g \in t^{-1}(y)} \varphi(g)\left(g \cdot\left(\sigma_{1}+\sigma_{2}\right)(s(g))\right) \\
& =\sum_{g \in t^{-1}(y)} \varphi(g)\left(g \cdot\left(\sigma_{1}(s(g))+\sigma_{2}(s(g))\right)\right) \\
& =\sum_{g \in t^{-1}(y)} \varphi(g)\left(g \cdot\left(\sigma_{1}(s(g))\right)+g \cdot\left(\sigma_{2}(s(g))\right)\right) \\
& =\sum_{g \in t^{-1}(y)} \varphi(g)\left(g \cdot\left(\sigma_{1}(s(g))\right)\right)+\sum_{g \in t^{-1}(y)} \varphi(g)\left(g \cdot\left(\sigma_{2}(s(g))\right)\right) \\
& =\left(\varphi \sigma_{1}\right)(y)+\left(\varphi \sigma_{2}\right)(y) .
\end{aligned}
$$

$\operatorname{Logo}\left(\varphi\left(\sigma_{1}+\sigma_{2}\right)\right)(y)=\left(\varphi \sigma_{1}\right)(y)+\left(\varphi \sigma_{2}\right)(y)$.

Por outro lado, dados $\varphi_{1}, \varphi_{2} \in C_{c}^{\infty}(G), \sigma \in \Gamma_{c}^{\infty}(E)$ e $y \in M$, temos que,

$$
\begin{aligned}
\left(\left(\varphi_{1}+\varphi_{2}\right) \sigma\right)(y) & =\sum_{g \in t^{-1}(y)}\left(\varphi_{1}+\varphi_{2}\right)(g)(g \cdot(\sigma(s(g))) \\
& =\sum_{g \in t^{-1}(y)}\left(\varphi_{1}(g)+\varphi_{2}(g)\right)(g \cdot(\sigma(s(g))) \\
& =\sum_{g \in t^{-1}(y)} \varphi_{1}(g)\left(g \cdot(\sigma(s(g)))+\sum_{g \in t^{-1}(y)} \varphi_{1}(g)(g \cdot(\sigma(s(g))) .\right.
\end{aligned}
$$

Portanto, $\left(\left(\varphi_{1}+\varphi_{2}\right) \sigma\right)(y)=\left(\varphi_{1} \sigma\right)(y)+\left(\varphi_{2} \sigma\right)(y)$.

ii) $\operatorname{Dados} \varphi, \psi \in C_{c}^{\infty}(G), \sigma \in \Gamma_{c}^{\infty}(E)$ e $y \in M$ :

$$
\begin{aligned}
((\varphi * \psi) \sigma)(y) & =\sum_{g \in t^{-1}(y)}(\varphi * \psi)(g)(g \cdot \sigma(s(g))) \\
& =\sum_{g \in t^{-1}(y)}\left(\sum_{g=g^{\prime} g^{\prime \prime}}\left(\varphi\left(g^{\prime}\right) \psi\left(g^{\prime \prime}\right)(g \cdot \sigma(s(g)))\right)\right. \\
& =\sum_{g^{\prime} \in t^{-1}(y)}\left(\varphi\left(g^{\prime}\right) \psi\left(g^{\prime \prime}\right)\left(\left(g^{\prime} g^{\prime \prime}\right) \cdot \sigma\left(s\left(g^{\prime \prime}\right)\right)\right)\right) .
\end{aligned}
$$


Por outro lado, temos que,

$$
\begin{gathered}
(\varphi(\psi \sigma))(y)=\sum_{g \in t^{-1}(y)} \varphi(g)\left(g \cdot((\psi \sigma)(s(g)))=\sum_{g^{\prime} \in t^{-1}(y)} \varphi\left(g^{\prime}\right)\left(g^{\prime} \cdot\left((\psi \sigma)\left(s\left(g^{\prime}\right)\right)\right)\right.\right. \\
=\sum_{g^{\prime} \in t^{-1}(y)} \varphi\left(g^{\prime}\right)\left(g^{\prime} \cdot\left(\sum_{g^{\prime \prime} \in t^{-1}\left(s\left(g^{\prime}\right)\right)} \psi\left(g^{\prime \prime}\right)\left(g^{\prime \prime} \cdot \sigma\left(s\left(g^{\prime \prime}\right)\right)\right)\right)\right. \\
=\sum_{g^{\prime} \in t^{-1}(y)} \varphi\left(g^{\prime}\right) \psi\left(g^{\prime \prime}\right)\left(g^{\prime} \cdot\left(g^{\prime \prime} \cdot \sigma\left(s\left(g^{\prime \prime}\right)\right)\right)\right)=\sum_{g^{\prime} \in t^{-1}(y)}\left(\varphi\left(g^{\prime}\right) \psi\left(g^{\prime \prime}\right)\left(\left(g^{\prime} g^{\prime \prime}\right) \cdot \sigma\left(s\left(g^{\prime \prime}\right)\right)\right)\right) .
\end{gathered}
$$

Portanto $((\varphi * \psi) \sigma)(y)=(\varphi(\psi \sigma))(y)$.

Assim, o espaço vetorial $\Gamma_{c}^{\infty}(E)$ das seções suaves de $E$ com suporte compacto possui uma estrutura natural de módulo sobre a álgebra de $C_{c}^{\infty}(G)$.

Todo morfismo $\phi: E \rightarrow F$ de representações de $G$ é em particular, um morfismo de fibrados vetoriais que cobre a identidade e portanto, induz um morfismo $\Gamma_{c}^{\infty}(\phi): \Gamma_{c}^{\infty}(E) \rightarrow \Gamma_{c}^{\infty}(F)$ de $C_{c}^{\infty}(M)$-módulos e considerando que a aplicação $\phi$ é linear nas fibras e $G$-equivariante obtemos que $\Gamma_{c}^{\infty}(\phi)$ é morfismo de $C_{c}^{\infty}(G)$-módulos. De fato,

$$
\left(\Gamma_{c}^{\infty}(\phi)(f \sigma)\right)(x)=\phi\left(\sum_{t(g)=x} f(g)(g \cdot \sigma(s(g)))\right)
$$

Como $\phi$ é $G$-invariante, temos

$$
\phi\left(\sum_{t(g)=x} f(g)(g \cdot \sigma(s(g)))=\sum_{t(g)=x} f(g)(g \cdot \phi(\sigma(s(g))))\right)=\left(f \Gamma_{c}^{\infty}(\phi)(\sigma)\right)(x) .
$$

Portanto, $\left(\Gamma_{c}^{\infty}(\phi)(f \sigma)\right)=\left(f \Gamma_{c}^{\infty}(\phi)(\sigma)\right)$, para toda $f \in C_{c}^{\infty}(G)$ e para todo $\sigma \in \Gamma_{c}^{\infty}(E)$.

A definição a seguir é uma generalização da definição de módulos projetivos. Lembre que todo $C_{c}^{\infty}(G)$-módulo é também um $C_{c}^{\infty}(M)$-módulo.

Definição 3.2.1. Dizemos que um $C_{c}^{\infty}(G)$-módulo $\mathcal{M}$ é de tipo finito se for isomorfo, como $C_{c}^{\infty}(M)$-módulo, a um submódulo de $C_{c}^{\infty}(M)^{k}$ para algum $k \in \mathbb{N}$, onde $C_{c}^{\infty}(M)^{k}$ é o produto cartesiano $k$ vezes de $C_{c}^{\infty}(M)$.

Dada $M$ uma variedade diferenciável. Considere $I_{x} C_{c}^{\infty}(M)$ o ideal da álgebra de funções de $C_{c}^{\infty}(M)$ que se anulam em $x \in M$. Para todo $x \in M$ denotamos

$$
C_{c}^{\infty}(M)(x):=C_{c}^{\infty}(M) / I_{x} C_{c}^{\infty}(M) .
$$

Fixado $x \in M$. Considere a aplicação,

$$
\begin{aligned}
F: C_{c}^{\infty}(M)(x) & \longrightarrow \mathbb{C} \\
{[f] } & \longmapsto f(x) .
\end{aligned}
$$


Sejam $f_{1}, f_{2} \in C_{c}^{\infty}(M)$ tais que $\left[f_{1}\right]=\left[f_{2}\right]$. Logo,

$$
\left(f_{1}-f_{2}\right) \in I_{x} C_{c}^{\infty}(M) \Longleftrightarrow\left(f_{1}-f_{2}\right)(x)=0 \Longleftrightarrow f_{1}(x)=f_{2}(x) \Longleftrightarrow f_{1}=f_{2} .
$$

Portanto, a aplicação $F$ está bem definida.

Considere $f_{1}, f_{2} \in C_{c}^{\infty}(M)$ tais que $F\left(\left[f_{1}\right]\right)=F\left(\left[f_{2}\right]\right)$. Temos,

$$
F\left(\left[f_{1}\right]\right)=F\left(\left[f_{2}\right]\right) \Longleftrightarrow f_{1}(x)=f_{2}(x) \Longleftrightarrow f_{1}=f_{2} .
$$

Portanto, a aplicação $F$ é injetora.

Agora, mostraremos que $F$ é sobrejetora. Dado $z \in \mathbb{C}$, seja $[f] \in C_{c}^{\infty}(M)(x)$ tal que $f$ é constante igual a $z$ em uma vizinhança $U$ de $x$. Então, tomamos $\bar{V} \subset U$ e $\bar{W} \subset V$, existe uma aplicação $\phi: M \longrightarrow \mathbb{R}$ tal que $\left.\phi\right|_{\bar{W}}=1$ e $\left.\phi\right|_{M \backslash V}=0$. Definimos, $\hat{f}: M \longrightarrow \mathbb{C}$ dada por,

$$
\hat{f}(y)= \begin{cases}\phi(y) f(y), & \text { se } y \in U \\ 0, & \text { caso contrário. }\end{cases}
$$

Assim, obtemos que $F$ é sobrejetora. Além disto, $F$ é um morfismo de álgebras. Portanto,

$$
C_{c}^{\infty}(M)(x) \simeq \mathbb{C}
$$

Dado $\mathcal{M}$ um $C_{c}^{\infty}(G)$-módulo de tipo finito. Para cada $x \in M$, considere o $C_{c}^{\infty}(M)$-submódulo $I_{x} \mathcal{M}:=I_{x} C_{c}^{\infty}(M) \cdot \mathcal{M}$, para cada $m \in \mathcal{M}$ denotamos

$$
[m]_{x} \in \mathcal{M}(x):=\mathcal{M} / I_{x} \mathcal{M}
$$

Como $\mathcal{M}$ um $C_{c}^{\infty}(G)$-módulo de tipo finito, por definição existe um morfismo $\phi: \mathcal{M} \longrightarrow$ $C_{c}^{\infty}(M)^{k}$ de $C_{c}^{\infty}(M)$-módulos injetor que induz o seguinte morfismo injetor de espaços vetoriais,

$$
\begin{gathered}
\bar{\phi}: \mathcal{M}(x) \longrightarrow C_{c}^{\infty}(M)^{k}(x) \simeq \mathbb{C}^{k} \\
{[m]_{x} \longmapsto[\phi(m)]_{x} .}
\end{gathered}
$$

Portanto, para todo $x \in M, \mathcal{M}(x)$ herda uma estrutura de $\mathbb{C}$-espaço vetorial e possui dimensão finita. Isto motiva a seguinte definição.

Definição 3.2.2. Dizemos que um $C_{c}^{\infty}(G)$-módulo $\mathcal{M}$ de tipo finito tem posto constante se a função a cada $x \in M$ associa a dimensão de $\mathcal{M}(x)=\mathcal{M} / I_{x} \mathcal{M}$ é constante.

O próximo exemplo mostra que, dada um fibrado vetorial $E$ sobre $M$ o espaço vetorial $\Gamma_{c}^{\infty}(E)$ é $C_{c}^{\infty}(G)$-módulo de tipo finito e posto constante.

Exemplo 3.2.1. Dado $E \rightarrow M$ um fibrado vetorial. O espaço vetorial $\Gamma_{c}^{\infty}(E)$ das seçôes suaves de E com suporte compacto é $C_{c}^{\infty}(G)$-módulo de tipo finito e posto constante. De fato, como $\Gamma_{c}^{\infty}(E)$ é finitamente gerado, podemos escolher $\sigma_{1}, \cdots, \sigma_{k}$ seções de $\Gamma_{c}^{\infty}(E)$ tais que $\left\{\sigma_{k}\right\}$ geram $\Gamma_{c}^{\infty}(E)$. Considere o fibrado trivial $F=M \times \mathbb{C}^{k} . O C_{c}^{\infty}(M)$-módulo $\Gamma_{c}^{\infty}(F)$ é livre com $k$ geradores, que denotaremos por $\alpha_{1}, \cdots, \alpha_{k}$. Definimos um morfismo de $C_{c}^{\infty}(M)$-módulos

$$
\psi: \Gamma_{c}^{\infty}(F) \longrightarrow \Gamma_{c}^{\infty}(E)
$$




$$
\alpha_{i} \longmapsto \sigma_{i}
$$

Como $\Gamma_{c}^{\infty}$ é pleno, existe um morfismo $\phi: F \longrightarrow E$ de fibrados vetorias tal que $\Gamma_{c}^{\infty}(\phi)=\psi$. Temos

$$
\left\{\begin{array}{l}
\Gamma_{c}^{\infty}(\phi) \alpha_{i}=\phi \circ \sigma_{i} \\
\psi\left(\alpha_{i}\right)=\sigma_{i}
\end{array}\right.
$$

Portanto, $\phi \circ \alpha_{i}=\sigma_{i}$ para todo $i$. Para cada $x \in M$ temos $\sigma_{i}$ está na imagem de $\phi$ e como $\left\{\sigma_{i}(x)\right\}_{i=1, \cdots, k}$ geram a fibra $E_{x}$, então $\phi$ é sobrejetora. Considere a seguinte sequência

$$
\operatorname{Ker} \phi \stackrel{i}{\longrightarrow} F \stackrel{\phi}{\longrightarrow} E \longrightarrow 0
$$

Como $\phi$ é sobrejetora, $\operatorname{Im} \phi=E$ então $\phi$ tem posto constante. Pela Proposição 1.2.2, Ker $\phi$ é um subfibrado de F. Portanto, temos uma sequência exata de fibrados vetoriais. Logo, $F \simeq \operatorname{Ker} \phi \oplus E$. Como $\Gamma_{c}^{\infty}$ é aditivo,

$$
\Gamma_{c}^{\infty}(F) \simeq \Gamma_{c}^{\infty}(\operatorname{ker} \phi) \oplus \Gamma_{c}^{\infty}(E)
$$

Além disto, $\Gamma_{c}^{\infty}(F) \simeq C_{c}^{\infty}(M)^{k}$ e obtemos $\Gamma_{c}^{\infty}(E)$ é isomorfo à um submódulo de $C_{c}^{\infty}(M)^{k}$. Portanto, $\Gamma_{c}^{\infty}(E)$ é $C_{c}^{\infty}(G)$-módulo de tipo finito. Segue da Proposição 1.3.1, que $\Gamma_{c}^{\infty}(E)$ é $C_{c}^{\infty}(G)$ módulo de tipo finito e posto constante.

Veremos agora que, dada $E$ uma representação de $G$, o espaço vetorial $\Gamma_{c}^{\infty}(E)$ é $C_{c}^{\infty}(G)$-módulo de tipo finito e posto constante.

Proposição 3.2.1. Sejam $G$ um grupoide de Lie étale sobre $M$ e E uma representação de $G$. O $C_{c}^{\infty}(G)$-módulo $\Gamma_{c}^{\infty}(E)$ é de tipo finito e posto constante.

Demonstração. De fato, como $C_{c}^{\infty}(M)$ é subalgebra de $C_{c}^{\infty}(G), \Gamma_{c}^{\infty}(E)$ é $C_{c}^{\infty}(M)$-módulo e para toda $f \in C_{c}^{\infty}(M)$ pela Proposição 3.1.1, temos $\tilde{f} \in C_{c}^{\infty}(G)$. Para toda $\sigma \in \Gamma_{c}^{\infty}(E), y \in M$,

$$
(\tilde{f} \sigma)(y)=\sum_{g \in t^{-1}(y)} \tilde{f}(g)(g \cdot(\sigma(s(g))))=\tilde{f}(\epsilon(y))(\epsilon(y) \cdot(\sigma(\epsilon(y))))=f(y)(\sigma(y))
$$

Portanto,

$$
(\tilde{f} \sigma)(y)=f(y) \sigma(y), \text { para toda } f \in C_{c}^{\infty}(M), \sigma \in \Gamma_{c}^{\infty}(E) \text { e } y \in M
$$

Ou seja,a ação de $C_{c}^{\infty}(G)$ em $\Gamma_{c}^{\infty}(E)$ coincide com a ação usual de $C_{c}^{\infty}(M)$ em $\Gamma_{c}^{\infty}(E)$. Além disto, $\Gamma_{c}^{\infty}(E)$ é $C_{c}^{\infty}(M)$-módulo e, pelo exemplo $3.2 .1, \Gamma_{c}^{\infty}(E)$ é $C_{c}^{\infty}(G)$-módulo de tipo finito e posto constante.

Note que, na equação (3.9) vemos um motivo pelo qual precisamos considerar seções com suporte compacto.

a próxima proposiçõ mostra que, se $\mathcal{M}$ é $C_{c}^{\infty}(G)$-módulo do tipo finito e posto constante então $\mathcal{M}$ é $C_{c}^{\infty}(M)$-módulo projetivo e finitamente gerado.

Proposição 3.2.2. Se $\mathcal{M}$ é um $C_{c}^{\infty}(G)$-módulo do tipo finito e posto constante, então $\mathcal{M}$ é um $C_{c}^{\infty}(M)$-módulo projetivo e finitamente gerado. 
Demonstração. Como $C_{c}^{\infty}(M)$ é subálgebra de $C_{c}^{\infty}(G)$, temos que $\mathcal{M}$ é $C_{c}^{\infty}(M)$-módulo do tipo finito e posto constante. Para cada $x \in M, \mathcal{M}(x)$ é $\mathbb{C}$-espaço vetorial. Além disso, $\mathcal{M}(x) \subset$ $C_{c}^{\infty}(M)^{k}(x)$ e como $C_{c}^{\infty}(M)^{k}(x) \simeq \mathbb{C}^{k}$, existe $\mathcal{M}^{c}(x) \subset C_{c}^{\infty}(M)^{k}(x)$ tal que $\mathcal{M}(x) \oplus \mathcal{M}^{c}(x)=$ $C_{c}^{\infty}(M)^{k}(x)$, onde $\mathcal{M}^{c}(x)$ denota o complementar de $\mathcal{M}^{c}(x)$ em $C_{c}^{\infty}(M)^{k}(x)$.

Para cada $x \in M$, definimos

$$
\begin{aligned}
\psi_{x}: \mathcal{M}(x) \oplus \mathcal{M}^{c}(x) & \longrightarrow \mathcal{M}(x) \oplus \mathcal{M}^{c}(x) \\
\left(\left[m_{1}\right]_{x}+\left[m_{2}\right]_{x}\right) & \longmapsto\left[m_{2}\right]_{x} .
\end{aligned}
$$

Como $\mathcal{M}(x) \oplus \mathcal{M}^{c}(x)=C_{c}^{\infty}(M)^{k}(x)$ e $C_{c}^{\infty}(M)^{k}(x) \simeq \mathbb{C}^{k}$, obtemos um isomorfismo de fibrados vetoriais dado por

$$
\begin{aligned}
\psi: M \times C_{c}^{\infty}(M)^{k}(x) & \longrightarrow M \times C_{c}^{\infty}(M)^{k}(x) \\
\left(x,\left[m_{1}\right]_{x}+\left[m_{2}\right]_{x}\right) & \longmapsto\left(x,\left[m_{2}\right]_{x}\right) .
\end{aligned}
$$

Note que, $\psi^{2}=\psi$. Portanto $\psi$ é uma projeção.

Para cada $x \in M$, temos $\mathcal{M}(x)=\operatorname{Ker}\left(\psi_{x}\right)$ e $\mathcal{M}^{c}(x)=\operatorname{Im}\left(\psi_{x}\right)$. Como $\mathcal{M}$ tem posto constante, pela Proposição 1.2.2, Ker $\psi$ e $\operatorname{Im} \psi$ são subfibrados do fibrado trivial $M \times \mathbb{C}^{k}$. Pelo Teorema de Serre-Swan,

$$
\Gamma_{c}^{\infty}(\operatorname{Im} \psi) \simeq \mathcal{M} .
$$

Portanto, $\mathcal{M}$ é $C_{c}^{\infty}(M)$-módulo projetivo e finitamente gerado.

As observações a seguir serão úteis na demonstração do Teorema de Serre-Swan para grupoides de Lie étale que será apresentada na próxima seção.

Observação 3.2.1. Para todo $[m]_{x} \in \mathcal{M}(x)$ e toda função $f \in C_{c}^{\infty}(M)$, a ação de $C_{c}^{\infty}(M)$ em $\mathcal{M}(x)$ é

$$
(f m)(x):=f(x)[m]_{x} .
$$

Em particular,

$$
(f m)(x)= \begin{cases}{[m]_{x},} & \text { se } f(x)=1 \\ 0, & \text { se } f(x)=0\end{cases}
$$

Observação 3.2.2. Seja $\varphi \in C_{c}^{\infty}(G)$ com suporte compacto em uma bisseção $U$ de um grupoide de Lie étale $G$. Para toda função $f \in C_{c}^{\infty}(M)$ com suporte compacto em s(U) temos

$$
\varphi * f=\left(f \circ\left(\sigma_{U}^{-1}\right)\right) * \varphi
$$

onde $f \circ\left(\sigma_{U}^{-1}\right) \in C_{c}^{\infty}(s(U))$ e $\sigma_{U}: s(U) \longrightarrow t(U)$ é um difeomorfismo. Definimos $F: M \longrightarrow \mathbb{C}$ dada por $F=\phi\left(f \circ\left(\sigma_{U}^{-1}\right)\right)$ tal que

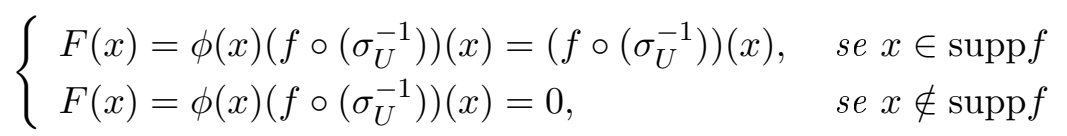


Por abuso de notação iremos usar $f \circ\left(\sigma_{U}^{-1}\right) \in C_{c}^{\infty}(M)$, ao nos referiremos à aplicação $F=$ $\phi\left(f \circ\left(\sigma_{U}^{-1}\right)\right)$. Portanto, $\varphi * f=\varphi$ se $f \equiv 1$ no conjunto $s(\operatorname{supp} \varphi) \subset s(U)$.

A observação 3.2.2 mostra que, se $G$ é um grupoide de Lie então a álgebra de convolução de $G$ tem unidades locais. Ou seja, para todo $\varphi \in C_{c}^{\infty}(G)$, existe $f \in C_{c}^{\infty}(G)$ tal que $\varphi * f=\varphi$.

\subsection{Teorema de Serre-Swan para grupoides de Lie étale}

Considere $\operatorname{Mod}(G)$ a categoria $\operatorname{dos} C_{c}^{\infty}(G)$-módulos de tipo finito e posto constante e $\operatorname{Mod}(M)$ a categoria dos $C_{c}^{\infty}(M)$-módulos. Dado $\mathcal{M} \in \operatorname{Mod}(G)$, como $C_{c}^{\infty}(M)$ é subálgebra de $C_{c}^{\infty}(G)$ temos $\mathcal{M} \in \operatorname{Mod}(M)$. Pela Proposição 3.2.2, $\mathcal{M}$ é $C_{c}^{\infty}(M)$-módulo é projetivo e finitamente gerado. Pelo Teorema de Serre-Swan, existe um fibrado vetorial $E$ sobre $M$ e um isomorfismo de $C_{c}^{\infty}(M)$-módulos $\phi: \Gamma_{c}^{\infty}(E) \longrightarrow \mathcal{M}$.

Para todo $x \in M, \mathcal{M}(x)$ é um $\mathbb{C}$-espaço vetorial. Logo,

$$
\phi_{x}: \Gamma_{c}^{\infty}(E) / I_{x} \Gamma_{c}^{\infty}(E) \longrightarrow \mathcal{M} / I_{x} \mathcal{M}
$$

é um isomorfismo induzido por $\phi$.

Como $\Gamma_{c}^{\infty}(E) / I_{x} \Gamma_{c}^{\infty}(E) \simeq E_{x}$ e $\mathcal{M} / I_{x} \mathcal{M} \simeq \mathcal{M}(x)$, obtemos uma aplicação bijetora e linear fibra a fibra

$$
\Phi: E \longrightarrow \coprod_{x \in M} \mathcal{M}(x)
$$

Portanto, $\pi: \coprod_{x \in M} \mathcal{M}(x) \longrightarrow M$ dada por $\pi\left([m]_{x}\right)=x$ é um fibrado vetorial. Note que, se existir outro fibrado vetorial $E^{\prime}$ e um isomorfismo de $C_{c}^{\infty}(M)$-módulos $\phi^{\prime}: \Gamma_{c}^{\infty}\left(E^{\prime}\right) \longrightarrow \mathcal{M}$, a seguinte composição

$$
\Gamma_{c}^{\infty}(E) \stackrel{\phi}{\longrightarrow} \mathcal{M} \stackrel{\phi^{\prime-1}}{\longrightarrow} \Gamma_{c}^{\infty}\left(E^{\prime}\right)
$$

é um isomorfismo de $C_{c}^{\infty}(M)$-módulos. Portanto, $E$ e $E^{\prime}$ são isomorfos e definem a mesma estrutura de fibrado vetorial em $\coprod_{x \in M} \mathcal{M}(x)$.

Mostraremos que o fibrado vetorial $\pi: \coprod_{x \in M} \mathcal{M}(x) \longrightarrow M$ é uma representação de $G$.

Seja $g \in G \operatorname{com} s(g)=x$ e $t(g)=y$. Considere $m \in \mathcal{M}$ tal que $[m]_{x} \in \mathcal{M}(x)$ e uma função $\varphi \in C_{c}^{\infty}(G)$ com suporte compacto contido em uma bisseção $U \subset G$ tal que $\varphi(g)=1$. Como $\mathcal{M}$ é um $C_{c}^{\infty}(G)$-módulo, $\varphi m \in \mathcal{M}$. Assim, definimos

$$
\begin{aligned}
\mu: G \times \coprod_{x \in M} \mathcal{M}(x) & \longrightarrow \coprod_{x \in M} \mathcal{M}(x) \\
\left(g,[m]_{x}\right) & \longmapsto[\varphi m]_{y}
\end{aligned}
$$

onde $[\varphi m]_{y} \in \mathcal{M}(y)$.

Mostraremos que $\mu$ está bem definida e $\mu$ é uma ação de $G$ em $\coprod_{x \in M} \mathcal{M}(x)$ linear em cada fibra. 
i) A aplicação $\mu$ não depende da escolha do representante.

Considere $m, m^{\prime} \in \mathcal{M}$ satisfazendo $[m]_{x}=\left[m^{\prime}\right]_{x}$ onde $x \in M$. Temos, $m-m^{\prime} \in I_{x} \mathcal{M}$. Para mostrarmos que $\mu$ independe da escolha do representante em $\mathcal{M}$, basta verificarmos que,

$$
g[m]_{x}=\left[g m^{\prime}\right]_{x} \text { ou, equivalentemente, }\left[g\left(m-m^{\prime}\right)\right]_{x}=\left[\varphi\left(m-m^{\prime}\right)\right]_{y}=0 .
$$

Como $m-m^{\prime} \in I_{x} \mathcal{M}$, existem $f_{i} \in I_{x} C_{c}^{\infty}(M)$ e $m_{i} \in \mathcal{M} \operatorname{com} i \in\{1, \ldots, k\}$ tais que

$$
m-m^{\prime}=\sum_{i=1}^{k} f_{i} m_{i}
$$

Seja $f^{\prime} \in C_{c}^{\infty}(M)$ com suporte compacto contido em $s(U)$ tal que $\left.f^{\prime}\right|_{s(\operatorname{supp} \varphi)} \equiv 1$. Pela observação 3.2 .2 , temos

$$
\varphi * f^{\prime}=\varphi
$$

Pela observação 3.2.2, a equação (3.11) e pela associatividade do produto de convolução, obtemos as seguintes igualdades:

$$
\left[g\left(m-m^{\prime}\right)\right]_{x}=\left[\varphi\left(m-m^{\prime}\right)\right]_{y}=\left[\varphi\left(\sum_{i=1}^{k} f_{i} m_{i}\right)\right]_{y}=\sum_{i=1}^{k}\left[\varphi\left(f_{i} m_{i}\right)\right]_{y}=\left(\sum_{i=1}^{k}\left[\varphi *\left(f^{\prime} f_{i}\right)\left(m_{i}\right)\right]\right)_{y}
$$

Usando (3.11) obtemos,

$$
\left.\sum_{i=1}^{k}\left[\varphi *\left(f^{\prime} f_{i}\right)\left(m_{i}\right)\right]\right)_{y}=\left[\sum_{i=1}^{k}\left(\varphi * f^{\prime}\right) *\left(f_{i}\right)\left(m_{i}\right)\right]_{y}
$$

E pela associatividade do produto de convolução e pela observação 3.2.2 temos,

$$
\left.\sum_{i=1}^{k}\left(\varphi * f^{\prime}\right) *\left(f_{i}\right)\left(m_{i}\right)\right]_{y}=\left[\sum_{i=1}^{k}\left(\left(f^{\prime} f_{i} \circ \sigma_{U}^{-1}\right) * \varphi\right)\left(m_{i}\right)\right]_{y}=\left[\sum_{i=1}^{k}\left(f^{\prime} f_{i} \circ \sigma_{U}^{-1}\right)\left(\varphi m_{i}\right)\right]_{y} .
$$

Como o suporte da aplicação $f^{\prime} f_{i}$ está contido em $s(U)$, para todo $i \in\{1, \ldots, k\}$ temos

$$
\left(f^{\prime} f_{i} \circ \sigma_{U}^{-1}\right)(y)=\left(f^{\prime} f_{i} \circ \sigma_{U}^{-1}\right)(t(g))=\left(f^{\prime} f_{i}(s(g))=f^{\prime}(s(g)) f(s(g)) .\right.
$$

Como $f^{\prime}(s(g)) f(s(g))=f^{\prime}(x) f(x)$, pois $x=s(g)$ e $f \in I_{x} C_{c}^{\infty}(M)$, pela observação 3.2.1, para todo $i \in\{1, \ldots, k\}$,

$$
\left(f^{\prime} f_{i} \circ \sigma_{U}^{-1}\right)\left(\left[\varphi m_{i}\right]_{y}\right)=0 \Longrightarrow\left[\varphi\left(m-m^{\prime}\right)\right]_{y}=0 .
$$

Com isto temos $[\varphi(m)]_{y}=\left[\varphi\left(m^{\prime}\right)\right]_{y}$, para todo $m, m^{\prime} \in \mathcal{M}$ tais que $[m]_{x}=\left[m^{\prime}\right]_{x}$ com $x \in M$. Isto prova que $\mu$ independe da escolha do representante em $\mathcal{M}$.

ii) A aplicação $\mu$ não depende da escolha do representante em $G$. Considere $\varphi, \varphi^{\prime} \in C_{c}^{\infty}(G)$ tais que o suporte compacto de $\varphi$ está contido em uma bisseção $U$ e o suporte compacto de $\varphi^{\prime}$ 
está contido em uma bisseção $U^{\prime}$ e $\varphi(g)=\varphi^{\prime}(g)=1$. Verificaremos que $[\varphi(m)]_{y}=\left[\varphi^{\prime}(m)\right]_{y}$. Considere $f, f^{\prime} \in C_{c}^{\infty}(M)$ tais que $f(y)=f^{\prime}(y)=1$, então

$$
f * \varphi=f^{\prime} * \varphi^{\prime}
$$

é uma função com suporte compacto contido na bisseção $U \cap U^{\prime}$. Pela observação 3.2.1,

$$
[\varphi m]_{y}=[f(\varphi m)]_{y}
$$

Pela equação $(3.12)$ e como $[f(\varphi m)]_{y}=[(f * \varphi)(m)]_{y}$, temos

$$
[(f * \varphi)(m)]_{y}=\left[\left(f^{\prime} * \varphi^{\prime}\right)(m)\right]_{y} .
$$

Pela associatividade do produto de convolução e pela observação 3.2.1,

$$
\left.\left.[(f * \varphi)(m)]_{y}=\left[f^{\prime}\left(\varphi^{\prime}(m)\right)\right)\right]_{y}=\left[\varphi^{\prime} m\right)\right]_{y} .
$$

Portanto, $[\varphi m]_{y}=\left[\varphi^{\prime} m\right]_{y}$ para toda $\varphi, \varphi^{\prime} \in C_{c}^{\infty}(G)$ tal que $\operatorname{supp} \varphi \subset U, \operatorname{supp} \varphi^{\prime} \subset U^{\prime}$ e $\varphi(g)=\varphi^{\prime}\left(g_{1}\right)=1$. Com isto provamos que $\mu$ independe da escolha do representante em $G$.

iii) A aplicação $\mu$ é uma ação suave. Dado $(g, m) \in G \times_{M} \coprod_{x \in M} \mathcal{M}(x)$ e $\varphi \in C_{c}^{\infty}(G)$ com suporte compacto contido na bisseção $U^{\prime}$ tal que $\left.\varphi\right|_{U} \equiv 1$ para alguma vizinhança $U \subset U^{\prime}$ de $g$. Seja $x \in M$ e considere $m_{1}, \ldots, m_{k} \in \mathcal{M}$ tais que $\left\{\left[m_{i}\right]_{x}\right\}_{i=1, \ldots, k}$ formam uma base para $\mathcal{M}(x)$. Para cada $\left[m_{i}\right]_{x} \in \mathcal{M}(x)$, existe $\sigma_{i} \in \Gamma_{c}^{\infty}\left(\coprod_{x \in M} \mathcal{M}(x)\right)$ tal que $\sigma_{i}(x)=\left[m_{i}\right]_{x}$. Então $\left\{\sigma_{i}(x)\right\}_{i=1, \ldots, k}$ é uma base para $\mathcal{M}(x)$ para todo $x \in s(U)$. Portanto, para todo $[m]_{x} \in \mathcal{M}(x)$ temos

$$
[m]_{x}=\sum_{i=1}^{k} a_{i}(m)\left[m_{i}\right]_{x}
$$

onde $a_{i}:\left.\coprod_{x \in M} \mathcal{M}(x)\right|_{s(U)} \longrightarrow \mathbb{C}$ são aplicações suaves e

$$
\left.\coprod_{x \in M} \mathcal{M}(x)\right|_{s(U)}=s^{*}(U)=\left\{(h, m) \in U \times \coprod_{x \in M} \mathcal{M} ; s(h)=\pi(m)\right\} .
$$

Agora dado $\left(h,[m]_{x}\right) \in U \times \coprod_{x \in M} \mathcal{M}(x)$ temos que,

$$
[h m]_{x}=h\left(\sum_{i=1}^{k} a_{i}(m)\left[m_{i}\right]_{x}\right)=\varphi\left(\sum_{i=1}^{k} a_{i}(m)\left[m_{i}\right]_{x}\right)=\varphi\left(\sum_{i=1}^{k} a_{i}(m)\left[m_{i}\right]_{t(h)}\right)=\sum_{i=1}^{k} a_{i}(m)\left[\varphi m_{i}\right]_{t(h)}
$$

Novamente, para cada $\left[\varphi m_{i}\right]_{t(h)} \in \mathcal{M}(t(h))$ existe $\alpha_{i} \in \Gamma_{c}^{\infty}\left(\coprod_{x \in M} \mathcal{M}(x)\right)$ tal que

$$
\left[\varphi m_{i}\right]_{t(h)}=\alpha_{i}(t(h)) .
$$

Isto prova que $\mu$ é suave. 
iv) Mostraremos que $\mu$ satisfaz as propriedades de uma ação e é linear em cada fibra. Se $\varphi, \varphi^{\prime} \in$ $C_{c}^{\infty}(G) \operatorname{com} \varphi(g)=\varphi\left(g_{1}\right)=1$ e $\operatorname{supp} \varphi \subset U$. Então

$$
\varphi^{\prime} * \varphi\left(g_{1} g\right)=\sum \varphi^{\prime}\left(g_{1}\right) \varphi(g)=1
$$

Temos que,

$$
\left(g_{1} g\right)[m]_{s\left(g_{1} g\right)}=\left[\left(\varphi^{\prime} * \varphi\right)(m)\right]_{t\left(g_{1} g\right)}=\left[\left(\varphi^{\prime}(\varphi m)\right)\right]_{t\left(g_{1} g\right)}
$$

Por outro lado,

$$
g_{1}\left([g m]_{(s(g))}=g_{1}\left([\varphi m]_{t(g))}=g_{1}\left([g m]_{(s(g))}=\left[\left(\varphi^{\prime}(\varphi m)\right)\right]_{t\left(g_{1} g\right)} .\right.\right.\right.
$$

Portanto, $\left(g_{1} g\right)[m]_{s\left(g_{1} g\right)}=g_{1}\left(g[m]_{s\left(g_{1} g\right)}\right)$, para todo $\left(g_{1}, g\right) \in G_{s} \times_{t} G$. Dado $g \in G$ com $s(g)=x, t(g)=y \operatorname{com} x, y \in M$ e $[m]_{x} \in \mathcal{M}(x)$,

$$
g[m]_{x}=(\varphi m)(y)=\left(\varphi\left(s^{-1}(y)\right)\right)(m(y))=m(y)
$$

Logo, $g[m]_{x}=m(y)$ e $\pi\left(g[m]_{x}\right)=\pi(m(y))=y=t(g)$ para todo $g \in G \operatorname{com} s(g)=x, t(g)=y$ $\operatorname{com} x, y \in M$ e $[m]_{x} \in \mathcal{M}(x)$.

A ação é linear em cada fibra. Dado $g \in G \operatorname{com} s(g)=x, t(g)=y \operatorname{com} x, y \in M$. Temos,

$$
\begin{aligned}
\mu_{g}: \mathcal{M}(x) & \longrightarrow \mathcal{M}(y) \\
{[m]_{x} } & \longmapsto g[m]_{x}
\end{aligned}
$$

Sejam $\left[m_{1}\right]_{x},\left[m_{2}\right]_{x} \in \mathcal{M}(x)$ arbitrários.

$$
\begin{aligned}
& \mu_{g}\left(\left[m_{1}\right]_{x}+\left[m_{2}\right]_{x}\right)=g\left(\left[m_{1}\right]_{x}+\left[m_{2}\right]_{x}\right)=g\left(\left[m_{1}+m_{2}\right]_{x}\right) \\
& \begin{aligned}
{\left[\varphi\left(m_{1}+m_{2}\right)\right]_{y} } & =\left[\varphi m_{1}+\varphi m_{2}\right]_{y}=\varphi\left(\left[m_{1}\right]_{y}\right)+\varphi\left(\left[m_{2}\right]_{y}\right) \\
& =\mu_{g}\left(\left[m_{1}\right]_{x}\right)+\mu_{g}\left(\left[m_{2}\right]_{x}\right) .
\end{aligned}
\end{aligned}
$$

Segue que, $\mu_{g}$ é linear em cada fibra. Portanto, $\coprod_{x \in M} \mathcal{M}(x)$ é uma representação de $G$.

Seja $G \rightrightarrows M$ um grupoide de Lie étale. Definimos

$$
\begin{aligned}
R_{G}: \operatorname{Mod}(G) & \longrightarrow \operatorname{Rep}(G) \\
\mathcal{M} & \longmapsto \coprod_{x \in M} \mathcal{M}(x) .
\end{aligned}
$$

Mostraremos que $R_{G}$ é um funtor.

Primeiramente, $R_{G}(\mathcal{M})=\coprod_{x \in M} \mathcal{M}(x)$ é uma representação de $G$, conforme mostramos acima. Dados $C_{c}^{\infty}(G)$ - módulos de tipo finito e posto constante $\mathcal{M}, \mathcal{N}$ e $\Phi: \mathcal{M} \longrightarrow \mathcal{N}$ morfismo de $C_{c}^{\infty}(G)$-módulos, $R_{G}(\Phi): R_{G}(\mathcal{M}) \longrightarrow R_{G}(\mathcal{N})$ é um morfismo de fibrados vetoriais $G$-invariante.

De fato, para todo $x \in M$ temos, 


$$
\begin{aligned}
\phi_{x}: \mathcal{M}(x) & \longrightarrow \mathcal{N}(x) \\
{[m]_{x} } & \longmapsto \phi_{x}\left([m]_{x}\right):=[\phi(m)]_{x} .
\end{aligned}
$$

A aplicação $\phi_{x}$ é linear fibra à fibra. Então,

$$
\begin{aligned}
R_{G}(\Phi):=\coprod_{x \in M} \phi_{x}: R_{G}(\mathcal{M}) & \longrightarrow R_{G}(\mathcal{N}) \\
{[m]_{x} } & \longmapsto[\Phi(m)]_{x} .
\end{aligned}
$$

Considere as projeções $\pi_{\mathcal{M}}: R_{G}(\mathcal{M}) \rightarrow M$ e $\pi_{\mathcal{N}}: R_{G}(\mathcal{N}) \rightarrow M$, dado $x \in M$, temos

$$
\left(\pi_{\mathcal{N}} \circ\left(R_{G}(\Phi)\right)\right)\left([m]_{x}\right)=\pi_{\mathcal{N}}\left([\Phi(m)]_{x}\right)=x=\pi_{\mathcal{M}}\left([m]_{x}\right)
$$

Portanto, $R_{G}(\mathcal{M})$ é um morfismo de fibrados vetoriais. Além disto, $R_{G}(\Phi)$ leva seções suaves do fibrado vetorial $R_{G}(\mathcal{M})$ em seções suaves do fibrado vetorial $R_{G}(\mathcal{N})$.

Resta verificar que, $R_{G}(\Phi)$ é $G$-invariante. Dado $g \in G$, com $s(g)=x$ e $t(g)=y$, onde $x, y \in M$. Considere $[m]_{x} \in \mathcal{M}(x)$ e $\varphi \in C_{c}^{\infty}(G)$ tal que $\operatorname{supp} \varphi \subset U$ sendo $U$ uma bisseção e $\varphi(g)=1$,

$$
\begin{aligned}
R_{G}(\Phi)\left(g\left([m]_{x}\right)\right)=R_{G}(\Phi)\left([\varphi m]_{y}\right) & =\phi_{y}\left([\varphi m]_{y}\right)=\left[\phi_{y}(\varphi m)\right]_{y}= \\
& =[\Phi(\varphi m)]_{y}=[\varphi(\Phi(m))]_{y}=g\left([\Phi(m)]_{x}\right)=g\left(\left(R_{G}(\Phi)\right)\left([m]_{x}\right)\right),
\end{aligned}
$$

com isto, concluímos que $R_{G}(\Phi)$ é $G$-invariante.

Portanto, $R_{G}(\phi)$ é um morfismo de fibrados vetoriais $G$-invariante. Segue que, $R_{G}$ é um funtor.

Teorema 3.3.1. (Serre-Swan para grupoides de Lie étale) Seja G um grupoide de Lie étale sobre uma variedade diferenciável $M$. O funtor

$$
\begin{aligned}
\Gamma_{c}^{\infty}: \operatorname{Rep}(G) & \longrightarrow \operatorname{Mod}(G) \\
E & \longmapsto \Gamma_{c}^{\infty}(E)
\end{aligned}
$$

é uma equivalência de categorias.

Demonstração. Esta equivalência de categorias será provada através de isomorfimos naturais, $\Gamma_{c}^{\infty} \circ R_{G} \simeq I d_{\operatorname{Mod}(G)}$ e $R_{G} \circ \Gamma^{\infty_{c}} \simeq I d_{\operatorname{Mod}(G)}$.

1) Existe um isomorfismo natural $\xi$ entre $\Gamma_{c}^{\infty} \circ R_{G}$ e $I d_{\operatorname{Mod}(G)}$ satisfazendo os itens i) e ii) a seguir.

i) Dado $\mathcal{M} \in \operatorname{Mod}(G)$, temos pelo Teorema de Serre Swan que $\Gamma_{c}^{\infty}\left(R_{G}(\mathcal{M})\right) \simeq \mathcal{M}$. Para todo $\mathcal{M} \in \operatorname{Mod}(G)$, definimos

$$
\begin{aligned}
\xi_{\mathcal{M}}: \mathcal{M} & \longrightarrow \Gamma_{c}^{\infty}\left(R_{G}(\mathcal{M})\right) \\
m & \longmapsto\left(x \stackrel{\sigma}{\longmapsto}[m]_{x}\right)
\end{aligned}
$$

Dados $m_{1}, m_{2} \in \mathcal{M}, \operatorname{temos} \xi\left(m_{1}\right)=\left(x \stackrel{\sigma_{1}}{\longmapsto}\left[m_{1}\right]_{x}\right)$ e $\xi\left(m_{2}\right)=\left(x \stackrel{\sigma_{2}}{\longmapsto}\left[m_{2}\right]_{x}\right)$,

$$
\xi\left(m_{1}+m_{2}\right)=\left(x \stackrel{\sigma}{\longmapsto}\left[m_{1}+m_{2}\right]_{x}\right)=\left(x \stackrel{\sigma}{\longmapsto}\left[m_{1}\right]_{x}+\left[m_{2}\right]_{x}\right)
$$




$$
\left(\left(x \stackrel{\sigma_{1}}{\longmapsto}\left[m_{1}\right]_{x}\right)+\left(x \stackrel{\sigma_{2}}{\longmapsto}\left[m_{2}\right]_{x}\right)\right)=\xi\left(m_{1}\right)+\xi\left(m_{2}\right) .
$$

Portanto, $\xi_{\mathcal{M}}$ é um isomorfismo de $C_{c}^{\infty}(G)$-módulos.

ii) Para todo morfismo de $C_{c}^{\infty}(G)$-módulos $\phi: \mathcal{M} \rightarrow \mathcal{N}$, o seguinte diagrama é comutativo

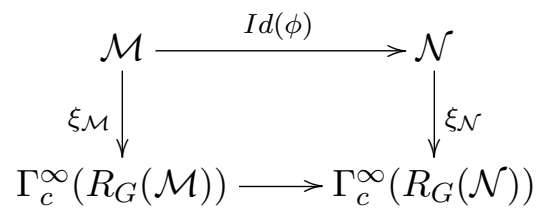

De fato, dado $m \in \mathcal{M}$,

$$
\left(\xi_{\mathcal{N}} \circ I d(\phi)\right)(m)=\left(\xi_{\mathcal{N}} \circ \phi\right)(m)=\xi_{\mathcal{N}}(\phi(m))=\left(x \stackrel{\sigma_{\mathcal{N}}}{\longmapsto}[\phi(m)]_{x}\right) .
$$

Por outro lado, $\left(\Gamma_{c}^{\infty}\left(R_{G}(\phi)\right)\right) \circ\left(\xi_{\mathcal{M}}\right)(m)=\left(R_{G}(\phi)\right)\left(x \stackrel{\sigma_{\mathcal{M}}}{\longmapsto}[m]_{x}\right)=\left(x \longmapsto[\phi(m)]_{x}\right)$. Portanto, temos que

$$
\left(\xi_{\mathcal{N}} \circ \operatorname{Id}(\phi)\right)(m)=\left(\Gamma_{c}^{\infty}\left(R_{G}(\phi)\right) \circ\left(\xi_{\mathcal{M}}\right)\right)(m)
$$

para todo $m \in \mathcal{M}$.

Assim, obtemos um isomorfismo natural $\xi$ entre os funtores $I d_{M o d(G)}$ e $\Gamma_{c}^{\infty} \circ R_{G}$.

2) Existe um isomorfismo natural entre $R_{G} \circ \Gamma_{c}^{\infty}$ e $I d_{\text {Mod }}$ satisfazendo os itens i) e ii) a seguir.

i) Dado $E$ uma representação de $G$, temos

$$
R_{G}\left(\Gamma_{c}^{\infty}(E)\right)=\coprod_{x \in M} \Gamma_{c}^{\infty}(E) / I_{x} \Gamma_{c}^{\infty}(E)
$$

Pela Proposição 1.3.1, segue-se que $\Gamma_{c}^{\infty}(E) / I_{x} \Gamma_{c}^{\infty}(E) \simeq E_{x}, \operatorname{logo}, R_{G}\left(\Gamma_{c}^{\infty}(E)\right) \simeq E$.

Para cada $E$ uma representação de $G$ e $x \in M$, definimos

$$
\begin{aligned}
\eta_{E}: R_{G}\left(\Gamma_{c}^{\infty}(E)\right) & \longrightarrow E \\
{[\sigma] } & \longmapsto \sigma(x)
\end{aligned}
$$

Observe que, $\eta_{E}$ é bem definida pela Proposição 1.3.1. Além disto, para todos $\left[\sigma_{1}\right],\left[\sigma_{2}\right] \in$ $R_{G}\left(\Gamma_{c}^{\infty}(E)\right)$, temos

$$
\eta\left(\left[\sigma_{1}\right]+\left[\sigma_{2}\right]\right)=\eta\left(\left[\sigma_{1}+\sigma_{2}\right]\right)=\left(\sigma_{1}+\sigma_{2}\right)(x)=\left(\sigma_{1}\right)(x)+\left(\sigma_{2}\right)(x)=\eta\left(\left[\sigma_{1}\right]\right)+\eta\left(\left[\sigma_{2}\right]\right)
$$

Logo, $\eta_{E}$ é um isomorfismo de representações de $G$.

ii) Dado um morfismo $\phi: E \rightarrow F$ entre representações de $G$, o seguinte diagrama é comutativo

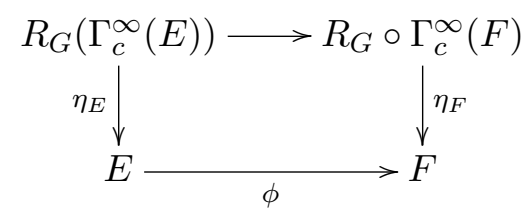


De fato, dado $[\sigma] \in R_{G}\left(\Gamma_{c}^{\infty}(E)\right)$, temos

$$
\phi\left(\eta_{E}([\sigma])\right)=\phi(\sigma(x))=(\phi \circ \sigma)(x)
$$

Por outro lado,

$$
\begin{aligned}
\eta_{F}\left(\left(R_{G}\left(\Gamma_{c}^{\infty}\right)(\phi)\right)([\sigma])\right) & =\left[\left(\left(R_{G}\left(\Gamma_{c}^{\infty}\right)(\phi)\right)([\sigma])\right)\right] \in R_{G}\left(\Gamma_{c}^{\infty}(F)\right) \\
& =\left(\left(R_{G}\left(\Gamma_{c}^{\infty}\right)(\phi)\right)(\sigma(x))\right) \\
& =\left(\left(\left(\Gamma_{c}^{\infty}\right)(\phi)\right)(\sigma)\right)(x) \\
& =(\phi \circ \sigma)(x) .
\end{aligned}
$$

Portanto, $\phi\left(\eta_{E}([\sigma])\right)=\eta_{F}\left(\left(R_{G}\left(\Gamma_{c}^{\infty}\right)(\phi)\right)([\sigma])\right)$, para todo $[\sigma] \in R_{G}\left(\Gamma_{c}^{\infty}(E)\right)$.

Assim, obtemos um isomorfismo natural $\eta$ entre os funtores $R_{G} \circ \Gamma_{c}^{\infty}$ e $I d_{\operatorname{Rep}(G)}$. O que conclui a demonstração. 


\section{Capítulo 4}

\section{Equivalência de Morita para álgebra de convolução de grupoides de Lie étale}

Neste capítulo mostraremos que se dois grupoides de Lie étale são Morita equivalentes então as categorias de módulos sobre suas as respectivas álgebras de convolução são isomorfas e que a subcategoria dos módulos de tipo finito e posto constante é preservada. Nossas principais referências serão [5] e [9].

\subsection{Equivalência de Morita e a categoria dos módulos}

O objetivo desta seção é provarmos que, se dois grupoides de Lie étale são Morita equivalentes então as categorias de módulos sobre suas respectivas álgebras de convolução são isomorfas.

Dados dois grupoides de Lie $G$ e $H$ Morita equivalentes, existe um $(G$ - $H)$-bifibrado $P$ tal que a ação de $G$ à esquerda é principal. O espaço vetorial $C_{c}^{\infty}(P)$ herda uma estrutura de $C_{c}^{\infty}(G)$ $C_{c}^{\infty}(H)$-bimódulo dada por,

$$
(\varphi \cdot f)(p)=\int_{g \in t^{-1}\left(a_{l}(p)\right)} \varphi(g) f\left(g^{-1} \cdot p\right) d \lambda^{a_{l}(p)}
$$

e

$$
(f \cdot \psi)(p)=\int_{h \in t^{-1}\left(a_{r}(p)\right)} f(p \cdot h) \psi\left(h^{-1}\right) d \beta^{a_{r}(p)},
$$

onde $\varphi \in C_{c}^{\infty}(G), \psi \in C_{c}^{\infty}(H), f \in C_{c}^{\infty}(P), p \in P$ e $\left\{\lambda^{a_{l}(p)}\right\}$ e $\left\{\beta^{a_{r}(p)}\right\}$ são sistemas de Haar de $G$ e $H$, respectivamente. Se $G$ e $H$ são grupoides de Lie étale, então a estrutura de $C_{c}^{\infty}(G)-C_{c}^{\infty}(H)$ bimódulo em $C_{c}^{\infty}(P)$ apresentada acima toma a forma:

$$
(\varphi \cdot f)(p)=\sum_{g \in t^{-1}\left(a_{l}(p)\right)} \varphi(g) f\left(g^{-1} \cdot p\right) \text { e }(f \cdot \psi)(p)=\sum_{h \in t^{-1}\left(a_{r}(p)\right)} f(p \cdot h) \psi\left(h^{-1}\right) .
$$

A partir de agora, assumiremos que $G \rightrightarrows M, H \rightrightarrows N$ e $K \rightrightarrows W$ são grupoides de Lie étale. E 
que, o $(G-H)$-bifibrado principal $P$ e o $(H-K)$-bifibrado principal $Q$ possuem as seguintes estruturas:

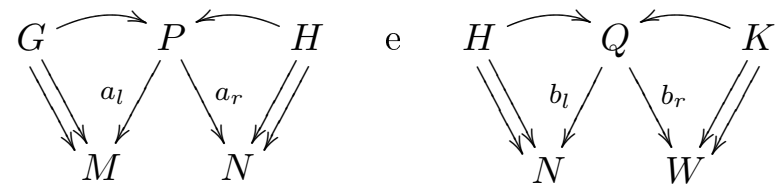

Dados abertos $U \in P, \tilde{U} \in Q$ abertos, consideraremos neste capítulo que as aplicações $\left.a_{r}\right|_{U}$ e $\left.b_{l}\right|_{\tilde{U}}$ são injetoras. Enunciaremos dois lemas que serão utilizados na demonstração do resultado principal desta seção, cujas demonstrações estão em [9]. Para ambos os lemas precisamos da seguinte definição.

Definição 4.1.1. Sejam $G \rightrightarrows M, H \rightrightarrows N$ e $K \rightrightarrows W$ grupoides de Lie étale. Considere $P$ um $(G-H)$-bifibrado principal e $Q$ um (H-K)-bifibrado principal. Dados $f \in C_{c}^{\infty}(P)$ e $\tilde{f} \in C_{c}^{\infty}(Q)$ escreveremos $f \otimes \tilde{f}$ para denotar um elemento em $C_{c}^{\infty}(P) \otimes_{C_{c}^{\infty}(H)} C_{c}^{\infty}(Q)$. Sejam $U$ e $\tilde{U}$ abertos em $P$ e $Q$, respectivamente. Dizemos que $(f, \tilde{f})$ é um par básico em $(U, \tilde{U})$ se $\operatorname{supp} f \subset U, \operatorname{supp} \tilde{f} \subset \tilde{U}$ e $b_{l}(\tilde{U}) \subset a_{r}(U)$. O par básico $(f, \tilde{f})$ é dito normalizado se $\left.\overline{a_{r}}(f)\right|_{b_{l}(\operatorname{supp} \tilde{f})} \equiv 1$, onde

$$
\overline{a_{r}}: C_{c}^{\infty}(P) \longrightarrow C_{c}^{\infty}(M)
$$

é dada por

$$
\overline{a_{r}}(f)(p)=\sum_{y \in a_{r}^{-1}(p)} f(y)
$$

Lema 4.1.1. Sejam $G \rightrightarrows M, H \rightrightarrows N$ e $K \rightrightarrows W$ grupoides de Lie étale. Considere $P$ um $(G-H)$ bifibrado principal, $Q$ um (H-K)-bifibrado principal e $U, \tilde{U}$ abertos de $P$ e $Q$ respectivamente.

i) Se $f \in C_{c}^{\infty}(P), \tilde{f} \in C_{c}^{\infty}(Q)$ possuem suporte em $U$ e $\tilde{U}$, respectivamente. Então existe um aberto $V \subset \tilde{U}$ e uma aplicação $\tilde{f}_{1} \in C_{c}^{\infty}(Q)$ com suporte em $V$ tal que $\left(f, \tilde{f}_{1}\right)$ é um par básico em $(U, V)$ e $f \otimes \tilde{f}=f \otimes \tilde{f}_{1}$.

ii) Para todo par básico $(f, \tilde{f})$ em $(U, \tilde{U})$, existe um par básico normalizado $\left(f_{1}, \tilde{f}_{1}\right)$ em $(U, \tilde{U})$, tal que $f \otimes \tilde{f}=f_{1} \otimes \tilde{f}_{1}$.

iii) Se $(f, \tilde{f})$ e $\left(f_{1}, \tilde{f}\right)$ são pares básicos normalizados em $(U, \tilde{U})$, então $f \otimes \tilde{f}=f_{1} \otimes \tilde{f}$.

$$
f k=f .
$$

Lema 4.1.2. Sejam $G \rightrightarrows M, H \rightrightarrows N$ e $K \rightrightarrows W$ grupoides de Lie étale. Considere $P$ um $(G$ $H)$-bifibrado principal, $Q$ um (H-K)-bifibrado principal e $U, \tilde{U}$ abertos de $P$ e $Q$ respectivamente. $\operatorname{Sejam}(f, \tilde{f})$ um par básico em $(U, \tilde{U})$ e $V$ um aberto de $Q$ tal que $b_{r}(\tilde{U}) \subset b_{r}(V)$. Então existem abertos $U_{i}$ de $P$, abertos $\tilde{U}_{i}$ de $V$ e pares básicos normalizados $\left(f_{i}, \tilde{f}_{i}\right)$ em $\left(U_{i}, \tilde{U}_{i}\right), i \in\{1, \ldots, n\}$, tais que

$$
f \otimes \tilde{f}=\sum_{i=1}^{n} f_{i} \otimes \tilde{f}_{i} .
$$

Sejam $G \rightrightarrows M, H \rightrightarrows N$ e $K \rightrightarrows W$ grupoides de Lie étale. Considere $P$ um $(G$ - $H)$-bifibrado principal e $Q$ um (H-K)-bifibrado principal. Definimos uma aplicação bilinear 


$$
\Lambda: C_{c}^{\infty}(P) \times C_{c}^{\infty}(Q) \longrightarrow C_{c}^{\infty}(P \otimes Q)
$$

dada por

$$
\Lambda(f, \tilde{f})(p \otimes q)=\sum_{h \in t^{-1}\left(a_{r}(p)\right)} f(p \cdot h) \tilde{f}\left(h^{-1} \cdot q\right)
$$

Como $\tilde{f} \in C_{c}^{\infty}(Q)$ possui suporte compacto e as $t$-fibras são discretas, existe apenas uma quantidade finita de $h \in t^{-1}\left(a_{r}(p)\right)$ com $\tilde{f}\left(h^{-1} \cdot q\right) \neq 0$, então o somatório em (4.3) é finito. Além disto, $\Lambda$ não depende da escolha de representante na classe $(p \otimes q) \in P \otimes Q$. Portanto, $\Lambda(f, \tilde{f})$ está bem definida em $P \otimes Q$. Portanto, define de fato uma aplicação de $C_{c}^{\infty}(P) \times C_{c}^{\infty}(Q)$ em $C_{c}^{\infty}(P \otimes Q)$.

Proposição 4.1.1. Sejam $G \rightrightarrows M, H \rightrightarrows N$ e $K \rightrightarrows W$ e $L \rightrightarrows W$ grupoides de Lie étale. Se $P$ um (G-H)-bifibrado principal, $Q$ um (H-K)-bifibrado principal e $R$ (K-L)-bifibrado principal então, para toda $f \in C_{c}^{\infty}(P), \tilde{f} \in C_{c}^{\infty}(Q), \tilde{f}^{\prime} \in C_{c}^{\infty}(R)$ e $p \in P$ temos

$$
\Lambda((f, \tilde{f}), \tilde{\tilde{f}})=\Lambda(f, \Lambda(\tilde{f}, \tilde{\tilde{f}}))
$$

Demonstração. Segue da associatividade para bifibrados apresentada em 2.4.4.

A aplicação $\Lambda$ induz uma aplicação bem definida em $C_{c}^{\infty}(P) \otimes_{C_{c}^{\infty}(H)} C_{c}^{\infty}(Q)$. Esta aplicação será denotada por $\Omega$.

Teorema 4.1.1. Sejam $G, H$ e $K$ grupoides de Lie étale. Considere $P$ um (G-H)-bifibrado principal e $Q$ um (H-K)-bifibrado principal. Então a aplicação

$$
\Omega:\left(C_{c}^{\infty}(P) \otimes_{C_{c}^{\infty}(H)} C_{c}^{\infty}(Q)\right) \longrightarrow C_{c}^{\infty}(P \otimes Q)
$$

dada por:

$$
\Omega(f \otimes \tilde{f})(p \otimes q)=\sum_{h \in t^{-1}\left(a_{r}(p)\right)} f(p \cdot h) \tilde{f}\left(h^{-1} \cdot q\right)
$$

é um isomorfismo de $C_{c}^{\infty}(G)-C_{c}^{\infty}(H)$-bimódulos.

Demonstração. Primeiramente, note que a Proposição 4.4 implica que $\Omega$ é um homomorfismo de $C_{c}^{\infty}(G)-C_{c}^{\infty}(\underset{\tilde{\tilde{f}}}{H})$-bimódulos. A aplicação $\Omega$ é sobrejetora. De fato, seja $\tilde{\tilde{f}} \in C_{c}^{\infty}(P \otimes Q)$. Podemos assumir que $\tilde{\tilde{f}}$ possui suporte compacto em $U \otimes \tilde{U}$, onde $U$ e $\tilde{U}$ são abertos de $P$ e $Q$ respectivamente, tais que $b_{l}(\tilde{U}) \subset a_{r}(U)$. Dado $q \in \tilde{U}$, temos $\left(\left.a_{r}\right|_{U}\right)^{-1}\left(b_{l}(q)\right) \in U$. Considere a aplicação

$$
\alpha: \tilde{U} \rightarrow U \otimes \tilde{U}
$$

dado por

$$
\alpha(q)=\left(\left(\left.a_{r}\right|_{U}\right)^{-1}\left(b_{l}(q)\right)\right) \otimes q .
$$

Como $\left.a_{r}\right|_{U}$ é injetora e $b_{l}$ é uma submersão sobrejetora, então $\alpha$ é um difeomorfismo.

Definimos,

$$
\begin{aligned}
& \tilde{f}: Q \longrightarrow \mathbb{C} \\
& q \longmapsto \tilde{\tilde{f}}(\alpha(q)),
\end{aligned}
$$


com suporte compacto em $\tilde{U}$, para todo $q \in Q$. Como $\tilde{\tilde{f}}, \alpha$ são suaves, temos que $\tilde{f}$ é suave. Portanto $\tilde{f} \in C_{c}^{\infty}(Q)$.

Como $S:=\left(\left.a_{r}\right|_{U}\right)^{-1}\left(b_{l}(\operatorname{supp} \tilde{f})\right)$ é um subconjunto compacto de $U$, podemos escolher $f \in C_{c}^{\infty}(P)$ com suporte compacto em $U$ tal que $\left.f\right|_{S} \equiv 1$, assim

$$
\Omega(f \otimes \tilde{f})=\tilde{\tilde{f}}
$$

Segue que, $\Omega$ é sobrejetora.

Mostraremos que a aplicação $\Omega$ é injetora. Seja $\eta \in C_{c}^{\infty}(P) \otimes_{C_{c}^{\infty}(H)} C_{c}^{\infty}(Q)$ tal que $\Omega(\eta)=0$. Pelo Lema 4.1.2 podemos escrever

$$
\eta=\sum_{i=1}^{n} f_{i} \otimes \tilde{f}_{i}
$$

onde $\left(f_{i}, \tilde{f}_{i}\right)$ é um par básico normalizado em $\left(U_{i}, \tilde{U}_{i}\right)$ e $U, \tilde{U}$ são abertos de $P$ e $Q$. Mostraremos que $\eta=0$. Vamos dividir o argumento em três casos.

i) No caso em que $n=1$, para todo $q \in \tilde{U}_{1}$ e $p \in P$ tal que $a_{r}(p)=b_{l}(q)$ temos

$$
0=\Omega(\eta)(p \otimes q)=f_{1}(p) \tilde{f}_{1}(q)
$$

Suponha que, $\tilde{f}_{1}(q) \neq 0$ para algum $q \in \tilde{U}_{1}$. Como $\left(f_{1}, \tilde{f}_{1}\right)$ é um par básico normalizado e $\tilde{f}_{1}(q) \neq 0$, podemos escolher $p \in a_{r}^{-1}\left(b_{l}(q)\right)$ tal que $f_{1}(p)=1$. Absurdo, então temos $\tilde{f}_{1}=0$ e portanto, $\eta=0$.

ii) Considere que existe um aberto $V$ em $P$ tal que $\tilde{U}_{i} \subset V$ para todo $i=1, \ldots, n$. Provaremos que $\eta=0$ por indução em $n$. O caso em que $n=1$, está verificado em $i$ ). A hipótese de indução será feita para $i=n-1$.

Denotaremos $S_{i}^{\prime}=\operatorname{supp} \tilde{f}_{i}, S_{i}=\left(\left.a_{r}\right|_{U_{i}}\right)^{-1}\left(b_{l}\left(S_{i}^{\prime}\right)\right), A_{i}=U_{n} \cap U_{i}$ e $C_{i}=S_{n} \cap S_{i}$, para todo $i=1, \cdots, n$.

Tomamos $A:=\bigcup_{i=1}^{n-1} A_{i}$ e $C:=\bigcup_{i=1}^{n-1} C_{i}$. Mostraremos que,

$$
b_{l}\left(S_{n}^{\prime}\right) \subset a_{r}(C) \subset a_{r}(A) .
$$

Como $C$ é compacto então $a_{r}(C)$ é fechado em $N$, basta verificarmos que $b_{l}(q) \in a_{r}(C)$, para todo $q \in S_{n}^{\prime} \operatorname{com} \tilde{f}_{n}(q) \neq 0$. Dado $q \in S_{n}^{\prime}$ e $p=\left(\left.a_{r}\right|_{U_{n}}\right)^{-1}\left(b_{l}(q)\right) \in \tilde{U}_{n}$, temos $p \in S_{n}^{\prime}$. Como $\Omega(\eta)=0$, então

$$
0=\Omega(\eta)(p \otimes q)=\sum_{i=1}^{n} f_{i}(p) \tilde{f}_{i}(q) .
$$

Como $\left(f_{n}, \tilde{f}_{n}\right)$ é um par básico normalizado e estamos assumindo que $\tilde{f}_{n}(q) \neq 0$, temos que $f_{n}(p)=1$. Portanto, existe $i \in\{1, \ldots, n-1\}$ tal que $f_{i}(p) \tilde{f}_{i}(q) \neq 0$. Logo, $p \in A_{i}$ e como $\tilde{f}_{n}(q) \neq 0$ temos $p \in S_{i}$. Com isto temos $p \in C$, o que resulta em $b_{l}(q) \in a_{r}(C)$. Isto prova as inclusões em (4.5).

Pelo Lema 4.1.1 iii) podemos assumir sem perda de generalidade que $\operatorname{supp} f_{n} \subset A \subset U_{n}$. Considere partições da unidade $\left\{\phi_{i}\right\}_{1 \leq i \leq n-1}$ em $b_{l}\left(S_{n}^{\prime}\right) \subset a_{r}(A)$ tais que $\phi_{i}: H \rightarrow[0,1]$ é suave com suporte compacto em $a_{r}\left(A_{i}\right)$. Em particular, $\sum_{i=1}^{n-1} \phi_{i}(b)=1$ para todo $b \in b_{l}\left(S_{n}^{\prime}\right)$. 
Definimos $\phi=\sum_{i=1}^{n-1} \phi_{i}$. Desta forma, temos que $\phi \in C_{c}^{\infty}(H)$ e obtemos

$$
\tilde{f}_{n}=\phi \cdot \tilde{f}_{n}=\sum_{i=1}^{n-1} \phi_{i} \cdot \tilde{f}_{n}
$$

e consequentemente, temos

$$
f_{n} \otimes \tilde{f}_{n}=\sum_{i=1}^{n-1} f_{n} \cdot \phi_{i} \otimes \tilde{f}_{n}
$$

onde $f_{n} \cdot \phi_{i}$ possui suporte compacto em $A_{i}$. Pelo Lema 4.1.1 $i$ ) e $i i$ ) encontramos abertos $B_{i} \subset \tilde{U}_{n}$ e pares básicos normalizados $\left(f_{i}, \tilde{f}_{i}\right)$ em $\left(A_{i}, B_{i}\right)$ tais que $f_{i} \otimes \tilde{f}_{i}=f_{n} \cdot \phi_{i} \otimes \tilde{f}_{n}$, para $i \in\{1, \ldots, n-1\}$. Como $A_{i} \subset U_{i},\left(l_{i}, \tilde{l}_{i}\right)$ é um par básico normalizado em $\left(U_{i}, B_{i} \cup \tilde{U}_{i}\right)$. Novamente pelo Lema 4.1.1 iii) podemos assumir sem perda de generalidade que $\left.f_{i}\right|_{\text {suppl }} \equiv 1$. Em particular, $l_{i} \otimes \tilde{l}_{i}=f_{i} \otimes \tilde{l}_{i}$. Logo,

$$
\eta=\sum_{i=1}^{n-1} f_{i} \otimes \tilde{f}_{i}+\sum_{i=1}^{n-1} f_{i} \otimes \tilde{l}_{i}=\sum_{i=1}^{n-1} f_{i} \otimes\left(\tilde{f}_{i}+\tilde{l}_{i}\right) .
$$

Note que, $\left(f_{i}, \tilde{f}_{i}+\tilde{l}_{i}\right)$ é um par básico normalizado em $\left(U_{i}, B_{i} \cup \tilde{U}_{i}\right)$ e $B_{i} \cup \tilde{U}_{i} \subset V$. Usando a hipótese de indução, concluímos que $\eta=0$.

iii) Considere uma partição da unidade $\left\{\psi_{i}\right\}_{\{i=1, \cdots, I\}}$ subordinada a $\left\{b_{r}\left(\tilde{U}_{i}\right)\right\}$.

Como $\bigcup_{i=1}^{n} b_{r}\left(\operatorname{supp} \tilde{f}_{i}\right) \subset \bigcup_{i=1}^{n} b_{r}\left(\tilde{U}_{i}\right)$, temos $\sum_{i=1}^{n} \psi_{i}(c)=1$ para todo $c \in \bigcup_{i=1}^{n} b_{r}\left(\operatorname{supp} \tilde{f}_{i}\right)$.

Tomamos $\phi=\sum_{i=1}^{n} \psi_{i} \in C_{c}^{\infty}(K)$. Portanto,

$$
\eta=\eta \cdot \phi=\sum_{i=1}^{n} \eta \cdot \psi_{i}=\sum_{j=1}^{n} \sum_{i=1}^{n} f_{i} \otimes \tilde{f}_{i} \cdot \psi_{j} .
$$

Para todo $1 \leq j \leq n$, temos $\Omega\left(\eta \cdot \psi_{j}\right)=\Omega(\eta) \cdot \psi_{j}=0$. Além disso, como $\tilde{f}_{i} \cdot \psi_{j}$ possui suporte compacto em $\tilde{U}_{i} \cap b_{r}^{-1}\left(b_{r}\left(\tilde{U}_{j}\right)\right)$ e $b_{r}\left(\tilde{U}_{i} \cap b_{r}^{-1}\left(b_{r}\left(\tilde{U}_{j}\right)\right)\right) \subset b_{r}\left(\tilde{U}_{j}\right)$ para todo $i$, basta tomarmos $V=\tilde{U}_{j}$ e obtemos $\eta \cdot \psi_{j}=0$, para todo $1 \leq j \leq n$. Assim, $\eta=0$. Com isto, obtemos que $\Omega$ é injetora. Portanto, $\Omega$ é um isomorfismo de $C_{c}^{\infty}(G)-C_{c}^{\infty}(H)$-bimódulos.

Seja $G \rightrightarrows M$ um grupoide de Lie. A categoria dos módulos sobre a álgebra de convolução $C_{c}^{\infty}(G)$ será denotada por $C_{c}^{\infty}(G)$-Mod.

Teorema 4.1.2. Se dois grupoides de Lie étale $G$ e $H$ são Morita equivalentes, então as categorias $C_{c}^{\infty}(G)$-Mod e $C_{c}^{\infty}(H)$-Mod são equivalentes.

Demonstração. Dados dois grupoides de Lie étale $G$ e $H$ Morita equivalentes, existe um $(G-H)$ bifibrado $P$ tal que a ação de $G$ à esquerda é principal. O espaço vetorial $C_{c}^{\infty}(P)$ herda uma estrutura de $C_{c}^{\infty}(G)$ - $C_{c}^{\infty}(H)$-bimódulo apresentada em (4.1). Além disto, ao invertemos as ações $C_{c}^{\infty}\left(P^{-1}\right)$ herda uma estrutura de $C_{c}^{\infty}(H)-C_{c}^{\infty}(G)$-bimódulo de maneira análoga, onde $P^{-1}$ é o bifibrado inverso de $P$. Definimos

$$
C_{c}^{\infty}(P) \otimes_{C_{c}^{\infty}(H)}-: C_{c}^{\infty}(H)-\operatorname{Mod} \longrightarrow C_{c}^{\infty}(G)-\operatorname{Mod}
$$




$$
\mathcal{M} \longmapsto C_{c}^{\infty}(P) \otimes_{C_{c}^{\infty}(H)} \mathcal{M}
$$

Note que, $C_{c}^{\infty}(P) \otimes_{C_{c}^{\infty}(H)} \mathcal{M}$ é um $C_{c}^{\infty}(G)$-módulo á esquerda.

De maneira análoga, definimos $C_{c}^{\infty}\left(P^{-1}\right) \otimes_{C_{c}^{\infty}(G)}-: C_{c}^{\infty}(G)$-Mod $\longrightarrow C_{c}^{\infty}(H)$-Mod.

Mostraremos que $C_{c}^{\infty}(P) \otimes_{C_{c}^{\infty}(H)}$ - é uma equivalência de categorias. Ou seja,

$$
\begin{aligned}
& \left(C_{c}^{\infty}(P) \otimes_{C_{c}^{\infty}(H)}-\right) \circ\left(C_{c}^{\infty}\left(P^{-1}\right) \otimes_{C_{c}^{\infty}(G)}-\right) \simeq \operatorname{Id}_{C_{c}^{\infty}(H)-\operatorname{Mod}} \\
& \left(C_{c}^{\infty}\left(P^{-1}\right) \otimes_{C_{c}^{\infty}(G)}-\right) \circ\left(C_{c}^{\infty}(P) \otimes_{C_{c}^{\infty}(H)}-\right) \simeq \operatorname{Id}_{C_{c}^{\infty}(G)-\operatorname{Mod}}
\end{aligned}
$$

Demonstraremos apenas (4.7), a demonstração de (4.8) é análoga.

Considere $\phi: \mathcal{M} \longrightarrow \mathcal{N}$ um morfismo de $C_{c}^{\infty}(H)$-módulos. Temos

$$
\begin{aligned}
& C_{c}^{\infty}(P) \otimes \phi: C_{c}^{\infty}(P) \otimes_{C_{c}^{\infty}(H)} \mathcal{M} \longrightarrow C_{c}^{\infty}(P) \otimes_{C_{c}^{\infty}(H)} \mathcal{N} \\
&(f \otimes m) \longmapsto(f \otimes \phi(m))
\end{aligned}
$$

A aplicação $C_{c}^{\infty}(P) \otimes \phi$ é um morfismo de $C_{c}^{\infty}(G)$-módulos. Dado $\mathcal{M}$ um $C_{c}^{\infty}(H)$-módulo temos pelo Teorema 4.1.1 que,

$$
\left(C_{c}^{\infty}\left(P^{-1}\right) \otimes_{C_{c}^{\infty}(H)} C_{c}^{\infty}(P)\right) \otimes \mathcal{M} \simeq\left(C_{c}^{\infty}\left(P^{-1} \otimes_{H} P\right)\right) \otimes_{C_{c}^{\infty}(H)} \mathcal{M}
$$

Como $P^{-1} \otimes_{H} P \simeq\left[I d_{H}\right]$ e $C_{c}^{\infty}\left(\left[I d_{H}\right]\right) \otimes_{C_{c}^{\infty}(H)} \mathcal{M} \simeq \mathcal{M}$ obtemos,

$$
\left(C_{c}^{\infty}\left(P^{-1}\right) \otimes_{C_{c}^{\infty}(H)} C_{c}^{\infty}(P)\right) \otimes_{C_{c}^{\infty}(H)} \mathcal{M} \simeq \mathcal{M} .
$$

Logo, $\left(C_{c}^{\infty}(P) \otimes_{C_{c}^{\infty}(H)}-\right) \circ\left(C_{c}^{\infty}\left(P^{-1}\right) \otimes_{C_{c}^{\infty}(G)}-\right) \simeq \operatorname{Id}_{C_{c}^{\infty}(H) \text {-Mod. De maneira análoga, }}$ $\left(C_{c}^{\infty}\left(P^{-1}\right) \otimes_{C_{c}^{\infty}(G)}-\right) \circ\left(C_{c}^{\infty}(P) \otimes_{C_{c}^{\infty}(H)}-\right) \simeq \operatorname{Id}_{C_{c}^{\infty}(G)-\operatorname{Mod}}$.

Portanto, o funtor $C_{c}^{\infty}(P) \otimes_{C_{c}^{\infty}(H)}$ - é uma equivalência entre a categoria $C_{c}^{\infty}(H)$-Mod e a categoria $C_{c}^{\infty}(G)$-Mod. O que conclui a demonstração.

Agora descreveremos alguns exemplos desta equivalência.

Exemplo 4.1.1. Considere os grupoides de Lie Morita equivalentes apresentados no exemplo (2.4.6), o grupoide do par $M \times M \rightrightarrows M$ é Morita equivalente ao grupoide $\{x\} \rightrightarrows\{x\}$. Se $M$ possui apenas $n$ elementos, então o grupoide do par é étale. Pelo Teorema 4.1.2, temos que a álgebra de convolução $C_{c}^{\infty}(M \times M \rightrightarrows M)$ é isomorfa à álgebra de convolução $C_{c}^{\infty}(\{x\} \rightrightarrows\{x\})$.

Exemplo 4.1.2. Considere o grupoide de transformação $G \ltimes M \rightrightarrows M$ tal que $G$ é um grupode de Lie discreto e a ação de $G$ em $M$ é livre e própria. Pelo exemplo 2.4.8, o grupoide de transformação $G \ltimes$ $M \rightrightarrows M$ é Morita equivalente ao grupoide unitário sobre o espaço de órbitas, $M / G \rightrightarrows M / G$. Pelo Teorema 4.1.2 temos que, a álgebra de convolução $C_{c}^{\infty}(G \ltimes M)$ é isomorfa à álgebra de convolução $C_{c}^{\infty}(M / G \rightrightarrows M / G)$. Pelo exemplo 3.1.5, $C_{c}^{\infty}(M / G \rightrightarrows M / G)=C_{c}^{\infty}(M / G)$.

Exemplo 4.1.3. O grupoide de submersão $N_{p} \times{ }_{p} N \rightrightarrows N$ é Morita equivalente ao grupoide unitário $M \rightrightarrows M$, conforme mostramos no exemplo 2.4.7. Considere ambos grupoides sendo grupoides étale, pelo Teorema 4.1.2 temos que, a álgebra de convolução $C_{c}^{\infty}\left(N_{p} \times_{p} N\right)$ é isomorfa à álgebra de convolução $C_{c}^{\infty}(M \rightrightarrows M)$. 


\subsection{Equivalência para módulos de tipo finito e posto constante}

O objetivo desta seção é mostrar usando o Teorema de Serre-Swan para grupoides de Lie étale, que o funtor $C_{c}^{\infty}(P) \otimes$, de fato preserva a subcategoria dos módulos de tipo finito e posto constante.

Proposição 4.2.1. Sejam $G \rightrightarrows M$ e $H \rightrightarrows N$ grupoides de Lie étale Morita equivalentes e seja $P$ $o(G-H)$ - bifibrado cuja ação de $G$ em $P$ é principal. Para toda $E$ uma representação de $H$ existe um isomorfismo natural de $C_{c}^{\infty}(G)$-módulos: $\delta_{P}(E): C_{c}^{\infty}(P) \otimes_{C_{c}^{\infty}(H)} \Gamma_{c}^{\infty}(E) \longrightarrow \Gamma_{c}^{\infty}\left(P \otimes_{H} E\right)$.

Demonstração. Considere $P$ um $(G-H)$-bifibrado cuja ação de $G$ em $P$ é principal e $E$ uma representação de $H$. Definimos a aplicação bilinear

$$
\beta_{P}(E): C_{c}^{\infty}(P) \times \Gamma_{c}^{\infty}(E) \longrightarrow \Gamma_{c}^{\infty}\left(P \otimes_{H} E\right)
$$

dada por

$$
\left(\beta_{P}(E)(f, \sigma)\right)(x)=\sum_{p \in a_{l}^{-1}(x)} f(p)\left(p \otimes \sigma\left(a_{r}(p)\right)\right)
$$

para toda $f \in C_{c}^{\infty}(P), \sigma \in \Gamma_{c}^{\infty}(E), x \in M$, onde $P \otimes_{H} E$ é a representação de $G$ apresentado em (2.20) e $a_{r}: P \longrightarrow N$ é a aplicação momento da ação de $H$ em $P \otimes_{H} E$. Note que, como $G$ e $H$ são étale, temos que $a_{l}^{-1}(x)$ é discreto. Além disso, como as funções $f, \sigma$ tem suporte compacto, concluímos que, para todo $x \in M$ temos uma quantidade finita de elementos em $\pi_{E}^{-1}(x)$ tal que $f(p) \neq 0$. Logo o somatório (4.9) é finito. Além disso, $\beta_{P}(E)$ é uma aplicação bilinear, então $\beta_{P}(E)$ induz o seguinte morfismo de $C_{c}^{\infty}(G)$-módulos

$$
\delta_{P}(E): C_{c}^{\infty}(P) \otimes_{C_{c}^{\infty}(H)} \Gamma_{c}^{\infty}(E) \longrightarrow \Gamma_{c}^{\infty}\left(P \otimes_{H} E\right) .
$$

Agora mostraremos que este morfismo $\delta_{P}(E)$ é bijetor.

Dadas funções suaves $\psi: G \rightarrow H$ e $\psi_{0}: M \rightarrow N$. Considere o caso em que $P=[\psi]$, ou seja $P$ é trivial. Por definição, $[\psi]:=M_{\psi_{0}} \times_{t} H$ é $(G-H)$-bifibrado, podemos considerar $P \otimes_{H} E$ uma representação de $G$, como apresentado em $(2.20)$. Seja $\psi^{*}(E)$ uma representação de $G$, onde $\psi^{*}(E):=M_{\psi_{0}} \times_{\pi_{E}} E$. Definimos,

$$
\begin{gathered}
\phi: P \otimes_{H} E \longrightarrow \psi^{*}(E) \\
([x, h], e) \longmapsto(x, e)
\end{gathered}
$$

A aplicação $\phi$ é um isomorfismo de representações de $G$. Em particular, $\phi$ é um isomorfismo de fibrados vetoriais sobre $M$, então obtemos o isomorfismo induzido de $C_{c}^{\infty}(G)$-módulos

$$
\begin{aligned}
\Gamma_{c}^{\infty}(\phi): \Gamma_{c}^{\infty}\left(P \otimes_{H} E\right) & \longrightarrow \Gamma_{c}^{\infty}\left(\psi^{*}(E)\right) \\
\sigma & \longmapsto \phi \circ \sigma .
\end{aligned}
$$

Como $E$ é uma representação de $H$, em particular é um fibrado vetorial sobre $N, C_{c}^{\infty}(N)$ é uma subalgebra de $C_{c}^{\infty}(H)$ e $C_{c}^{\infty}(M)$ é uma subalgebra de $C_{c}^{\infty}(G)$, temos um morfismo de álgebras induzido por $\delta_{P}(E)$,

$$
\delta_{P}^{\prime}(E): C_{c}^{\infty}(M) \otimes_{C_{c}^{\infty}(N)} \Gamma_{c}^{\infty}(E) \longrightarrow \Gamma_{c}^{\infty}\left(\psi^{*}(E)\right)
$$

A aplicação $\delta_{P}^{\prime}(E)$ é definido de maneira análoga a $\delta_{P}(E)$. 
Afirmamos que $\delta_{P}^{\prime}(E)$ é um isomorfismo de $C_{c}^{\infty}(M)$-módulos. Para mais detalhes, veja [10].

Como $P=M \otimes_{N} H$, pelo Teorema 4.1.1 temos $C_{c}^{\infty}(P) \simeq C_{c}^{\infty}(M) \otimes_{C_{c}^{\infty}(N)} C_{c}^{\infty}(H)$, é um $C_{c}^{\infty}(M)-C_{c}^{\infty}(H)$-bimódulo. Assim, escrevemos o seguinte isomorfimo de $C_{c}^{\infty}(M)$-módulos,

$$
\begin{gathered}
\left(C_{c}^{\infty}(M) \otimes C_{c}^{\infty}(H)\right) \otimes_{C_{c}^{\infty}(H)} \Gamma_{c}^{\infty}(E) \longrightarrow C_{c}^{\infty}(M) \otimes_{C_{c}^{\infty}(N)} \Gamma_{c}^{\infty}(E) \\
((f \otimes \varphi) \otimes \sigma) \longmapsto(f \otimes \sigma)
\end{gathered}
$$

Agora, construímos o seguinte diagrama comutativo

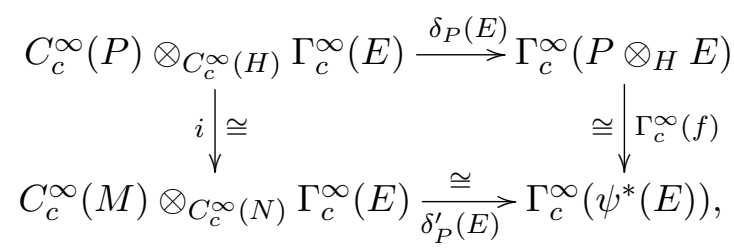

como as aplicações $i, \delta_{P}^{\prime}(E)$ e $\Gamma_{c}^{\infty}(\phi)$ são bijetoras, segue-se que $\delta_{P}(E)$ é bijetora.

Seja $P$ um $(G-H)$-fibrado principal. Assumindo que, $P$ é localmente trivial, seja $U$ um aberto de $M$ tal que $\left.P\right|_{U}$ é $H$-fibrado trivial, como descrito no início desta demonstração. Então temos um morfismo injetor de $C_{c}^{\infty}(H)$-módulos

$$
j: C_{c}^{\infty}\left(\left.P\right|_{U}\right) \longrightarrow C_{c}^{\infty}(P) .
$$

Como todo módulo é, em particular, um grupo abeliano, temos um morfismo injetor de grupos abelianos

$$
j \otimes i d: C_{c}^{\infty}\left(\left.P\right|_{U}\right) \otimes_{C_{c}^{\infty}(H)} \Gamma_{c}^{\infty}(E) \longrightarrow C_{c}^{\infty}(P) \otimes_{C_{c}^{\infty}(H)} \Gamma_{c}^{\infty}(E) .
$$

Para todo $w:=\sum f_{k} \otimes \sigma_{k} \in C_{c}^{\infty}\left(\left.P\right|_{U}\right) \otimes_{C_{c}^{\infty}(H)} \Gamma_{c}^{\infty}(E)$ da observação 3.2.2, temos que existe uma função $f \in C_{c}^{\infty}(M)$ com suporte em $U$ tal que $f f_{k}=f k$ para todo $k$. Por outro lado, a ação à esquerda por $f$

$$
\mu_{f}: C_{c}^{\infty}(P) \otimes_{C_{c}^{\infty}(H)} \Gamma_{c}^{\infty}(E) \longrightarrow C_{c}^{\infty}\left(\left.P\right|_{U}\right) \otimes_{C_{c}^{\infty}(H)} \Gamma_{c}^{\infty}(E)
$$

é um morfismo de grupos abelianos tal que $\mu_{f}((i \otimes i d)(w))=w$. Com isto, obtemos o seguinte diagrama,

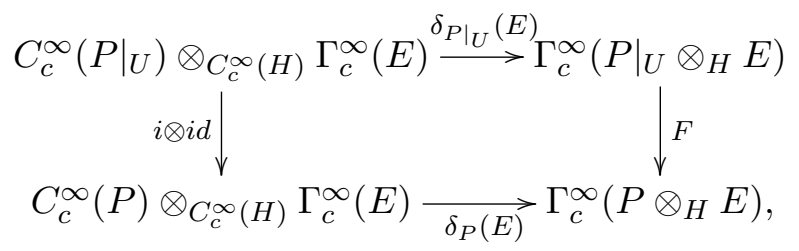

onde $F$ é definida estendendo as seções fora do aberto $U$ e as aplicações verticais são injetoras. Mostraremos agora que, $\delta_{P}(E)$ é inejtiva. De fato, seja $v \in C_{c}^{\infty}(P) \otimes_{C_{c}^{\infty}(H)} \Gamma_{c}^{\infty}(E)$ tal que $\delta_{P}(E)(v)=0$. Da observação 3.2.2, segue que o módulo $C_{c}^{\infty}(P)$ possui unidades locais, então existe uma função $f \in C_{c}^{\infty}(M)$ tal que $f v=v$ e escrevemos $f=\sum f_{i}$, onde $f_{i}$ são funções com suportes contidos em 
$U_{i}$ tal que $\left.P\right|_{U_{i}}$ é trivial para todo $i$. Como $\delta_{P}(E)$ é bilinear, temos

$$
\delta_{\left.P\right|_{U_{i}}}(E)\left(f_{i} v\right)=\delta_{P}(E)\left(f_{i} v\right)=f_{i} \delta_{P}(E)(v)=0
$$

Como $\delta_{\left.P\right|_{U}}(E)$ é injetiva, temos $f_{i} v=0$, para todo $i$. Portanto, $v=0$. Isto prova que $\delta_{P}(E)$ é inejtora. A demonstração de que $\delta_{P}(E)$ é sobrejetora é análoga ao que fizemos para provar a sua injetividade.

Teorema 4.2.1. Se dois grupoides de Lie étale $G$ e $H$ são Morita equivalentes, então as categorias $\operatorname{Mod}(G)$ e $\operatorname{Mod}(H)$ são equivalentes.

Demonstração. Dados $G$ e $H$ dois grupoides de Lie étale Morita equivalentes. Pela definição de equivalência de Morita, sabemos que existe um $(G-H)$-bifibrado $P$ tal que a ação de $G$ à esquerda em $P$ é principal. Definimos o funtor

$$
\operatorname{Mod}(P):=C_{c}^{\infty}(P) \otimes_{C_{c}^{\infty}(H)}-: \operatorname{Mod}(H) \longrightarrow \operatorname{Mod}(G)
$$

Ou seja, $\operatorname{Mod}(P)$ é a restrição do funtor apresentado no Teorema 4.1.2. Dado $\mathcal{M} \in \operatorname{Mod}(H)$, pelo Teorema de Serre-Swan para grupoides de Lie étale temos

$$
C_{c}^{\infty}(P) \otimes_{C_{c}^{\infty}(H)} \mathcal{M} \simeq C_{c}^{\infty}(P) \otimes_{C_{c}^{\infty}(H)} \Gamma_{c}^{\infty}\left(R_{G}(\mathcal{M})\right)
$$

Pela Proposição 4.2.1,$C_{c}^{\infty}(P) \otimes_{C_{c}^{\infty}(H)} \Gamma_{c}^{\infty}\left(R_{G}(\mathcal{M})\right)$ é um $C_{c}^{\infty}(G)$-módulo de tipo finito e posto constante. Portanto, o $\operatorname{Mod}(P)$ é um funtor bem definido. O fato de que, este funtor define uma equivalência de categorias, segue tomando o $(H-G)$-bifibrado inverso $P^{-1}$ e considerando o funtor $\operatorname{Mod}\left(P^{-1}\right): \operatorname{Mod}(H) \longrightarrow \operatorname{Mod}(G)$ definido de maneira análoga ao funtor $\operatorname{Mod}(P)$. Para todo $\mathcal{M} \in \operatorname{Mod}(H)$,

$$
\operatorname{Mod}\left(P^{-1}\right) \circ \operatorname{Mod}(P)(\mathcal{M}) \simeq C_{c}^{\infty}\left(P^{-1}\right) \otimes_{C_{c}^{\infty}(H)} C_{c}^{\infty}(P) \otimes_{C_{c}^{\infty}(H)} \mathcal{M} \simeq C_{c}^{\infty}\left(P^{-1} \otimes_{H} P\right) \otimes_{C_{c}^{\infty}(H)} \mathcal{M}
$$

Como $P^{-1} \otimes P \simeq\left[I d_{H}\right]$ e $C_{c}^{\infty}\left(\left[I d_{H}\right]\right) \otimes \mathcal{M} \simeq \mathcal{M}$, obtemos

$$
\operatorname{Mod}\left(P^{-1}\right) \circ \operatorname{Mod}(P) \simeq\left[I d_{H}\right]
$$

De maneira análoga,

$$
\operatorname{Mod}(P) \circ \operatorname{Mod}\left(P^{-1}\right) \simeq\left[I d_{G}\right]
$$

Portanto, o funtor $\operatorname{Mod}(P)$ é uma equivalência entre a categoria $\operatorname{Mod}(H)$ e a categoria $\operatorname{Mod}(G)$. O que conclui a demonstração. 


\section{Referências Bibliográficas}

[1] M. Crainic. Lectures on integrability of lie brackets. arXiv:math, 0611259:7-29, 2006.

[2] M. del Hoyo. Lie groupoids and their orbispaces. arXiv:math, 1212.6714v2, 2013.

[3] E.Lerman. Orbifolds as stacks? arxiv.org, 0806.4160v2, 2009.

[4] J.Kalisnik. Representations of orbifolds groupoids. arXiv:math, 0709.0176v2, 2007.

[5] J.Kalisnik. Groupoid representations and modules over the hopf algebroid. Czechoslovak Mathematical Journal, 61, 2011.

[6] K. Mackenzie. General theory of lie groupoids and lie algebroids. Lecture Notes London Math. Soc., 213, 2005.

[7] I. Moerdijk. Foliation groupoids and their cyclic homology. Advances in Mathematics, 1572:177-197, 2000.

[8] I. Moerdijk. Introduction to foliations and lie groupoids. Cambridge studies in advanced mathematics - Cambridge University, 91:92-148, 2003.

[9] J. Mrčun. Functoriality of the bimodule associated to a hilsum-skandalis map. Mathematics Subjet Classifications, 2005.

[10] J. Mrčun. Lie groupoids, sheaves and cohomology. London Math. Soc. Lecture Note Ser. 323, Cambridge University Press, 91(4):145-272, 2005.

[11] J. Renault. A groupoid approach to c*-algebras. Lecture Notes Springer-Verlag, 793, 1980.

[12] R. Swan. Vector bundles and projective modules. Trans. Amer. Math. Soc., 105, 1962.

[13] A. Weinstein. Geometric models for noncommutative algebras. Berkeley Mathematics Lecture Notes series, American Mathematical Society, 1999. 